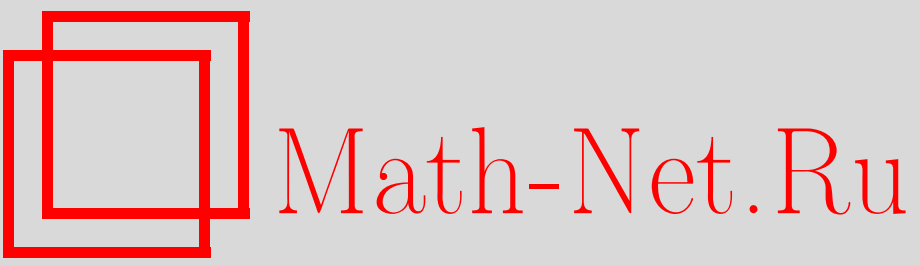

М. С. Агранович, Спектральные задачи для сильно эллиптических систем второго порядка в областях с гладкой и негладкой границей, УМН, 2002, том 57, выпуск 5, 3-78

DOI: https://doi.org/10.4213/rm552

Использование Общероссийского математического портала Math-Net.Ru подразумевает, что вы прочитали и согласны с пользовательским соглашением

http: //www. mathnet.ru/rus/agreement

Параметры загрузки:

IP : 3.81 .55 .215

26 апреля 2023 г., 13:22:05 


\title{
СПЕКТРАЛЬНЫЕ ЗАДАЧИ ДЛЯ СИЛЬНО \\ ЭЛЛИПТИЧЕСКИХ СИСТЕМ ВТОРОГО ПОРЯДКА В ОБЛАСТЯХ С ГЛАДКОЙ И НЕГЛАДКОЙ ГРАНИЦЕЙ
}

\author{
М. С. АГРАновИч
}

Рассматриваются спектральные задачи с дискретным спектром для сильн эллиптических систем уравнений в частных производных второго порядка в $n$-мерной области, граница $\Gamma$ которой компактна и может быть бесконечно гладкой, класса $C^{1,1}$ или липшицевой. Главная часть системы предполагается эрмитовой и подчиняется дополнительному условию, обеспечивающему коэрцитивность задачи Неймана. Спектральный параметр содержится или в системе (тогда рассматривается ограниченная область $\Omega$ ), или в граничном условии первого порядка. Рассматриваются также задачи в $\mathbb{R}^{n} \backslash \Gamma$ со спектральным параметром в условии сопряжения на $\Gamma$. Соответствующие операторы в $L_{2}(\Omega)$ или в $L_{2}(\Gamma)$ могут быть самосопряженными или близкими к самосопряженным. При некоторых дополнительных предположениях обсуждаются свойства гладкости, полноты и базисности собственных или корневых функций в соболевских $L_{2}$-пространствах $H^{t}(\Omega)$ и $H^{t}(\Gamma)$ ненулевого порядка $t$, а также локализация и асимптотика собственных значений. Охвачен случай кулоновских особенностей в младшем члене системы.

Библиограффия: 129 названий.

\section{СОДЕРЖАНИЕ}

Введение . . . . . . . . . . 4

Глава I. Задачи со спектральным параметром в системе ............. 9

$\S 1$. Задачи в области с гладкой границей $\ldots \ldots \ldots \ldots \ldots \ldots \ldots \ldots \ldots \ldots \ldots . \ldots . \ldots \ldots$

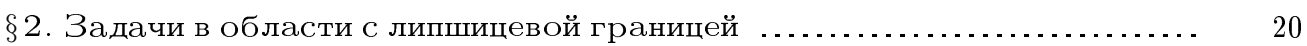

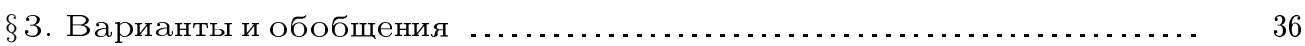

Глава II. Задачи со спектральным параметром в граничном условии или условии сопряжения ...................................... 43

$\S 4$. Задачи со спектральным параметром на гладкой границе .......... 43

$\S 5$. Задачи со спектральным параметром на липшицевой границе ....... 58

$\S 6$. Добавление к главе II. Некоторые спектральные задачи для систем Максвелла и Дирака .......................................... 68

$\S 7$. Заключительные замечания ...................................... 71

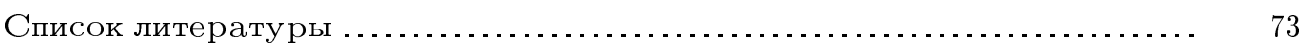

Работа выполнена при финансовой поддержке Российского фонда фундаментальных исследований (грант № 01-01-00284). 


\section{Введение}

1. Рамки статьи. Пусть $\Omega=\Omega^{+}$- ограниченная область в $\mathbb{R}^{n}, n \geqslant 2$, с $(n-1)$ мерной границей $\Gamma$, которую сначала будем считать гладкой. Мы объясним здесь рассматриваемые в статье вопросы на примере скалярного уравнения Шрёдингера

$$
-\Delta u(x)+V(x) u(x)=\lambda u(x)
$$

в $\Omega$ с потенциалом $V(x)$, которьй сначала тоже будем считать гладким и, кроме того, вешественным. Через $\nu=\nu(x)$ будем обозначать единичньй вектор внешней нормали в точке $x \in \Gamma$ и через $\partial_{\nu}-$ производную в направлении этого вектора. На $Г$ поставим граничное условие

$$
\partial_{\nu} u^{+}(x)+\mu u^{+}(x)=0,
$$

где значки ${ }^{+}$указьвают, что берутся предельные значения из $\Omega^{+}$. В (1) и (2) $\lambda$ и $\mu$ - два числовых параметра. Фиксируя значение второго параметра, получаем задачу со спектральным параметром $\lambda$ в уравнении. Фиксируя, наоборот, значение первого параметра, получаем задачу со спектральным параметром $\mu$ в граничном условии.

Эти простейшие спектральные задачи - типичные и хорошо известные примеры из двух классов аналогичных спектральных задач для сильно эллиптических систем второго порядка, и настояшая статья содержит обзор соответствуюших результатов и методов исследования. Мы начинаем с задач в области с гладкой границей, но затем переходим к задачам в липшицевых областях, именно здесь за последнее время имел место прогресс, заслуживаюший отражения в обзоре. При этом мы рассматриваем и некоторые новые вопросы, сравнивая результаты и способы их получения при разных предположениях о гладкости или негладкости границы. В п. 7.1 мы подведем некоторьй итог продвижениям в настоящей статье.

Понятие сильной эллиптичности ввел, как известно, М. И. Вишик в [125]. Дополнительные условия, которые мы наложим на главный символ системы, формулируются в п. 1.1 и п. 4.1. Наиболее интересные для нас примеры систем приведены в п. 1.2.

Кроме задач двух упомянутых типов, мы затронем внешние задачи в области $\Omega^{-}$, дополнительной к замьканию $\bar{\Omega}$ области $\Omega=\Omega^{+}$, со спектральным параметром в граничных условиях и задачи сопряжения в объединении областей $\Omega^{+}$и $\Omega^{-}$со спектральным параметром в условиях сопряжения. Система в этих задачах считается имеющей постоянные коэффициенты, по крайней мере, в окрестности бесконечности. Такие задачи дополняются условиями, накладываемыми на решения на бесконечности.

Если граница гладкая, то эти спектральные задачи исследуются средствами теории эллиптических граничных задач и эллиптических ПДО - псевдодифференциальных операторов - в сочетании с классическими методами теории потенциала. В обеих главах I и II мы сначала должны рассмотреть случай гладкой границы (в $\S 1$ и $\S 4)$. При этом мы будем предполагать, что читатель имеет некоторое знакомство с теорией эллиптических ПДО на гладкой поверхности и эллиптических задач в ограниченной области с гладкой границей в соболевских $L_{2}$-пространствах $H^{t}=W^{2, t}$. Для справок можно использовать обзоры [4], [8], а также книги [46].

Основные свойства соболевских пространств $H^{t}$ в липшицевых областях и на их гранищах мы напомним в п. 2.1. Пространствами $W^{p, t}$ с $p \neq 2$ в этой статье мы пользоваться не будем. Но в п. 2.4 дополнительно понадобятся два $L_{2}$-пространства Бесова, они определяются в п. 2.3. В нескольких пунктах от читателя предполагается 
некоторое знакомство с интерполяцией банаховых пространств; для справок можно использовать книгу [17].

Мы также остановимся на случае границы малой гладкости, а именно границы класса $C^{1,1}$ (его определение см. в п. 1.5), для которого сохраняются наиболее важные результаты, относяшиеся к областям с гладкой границей.

Затем мы будем подробно рассматривать менее простой случай липшицевых областей. Напомним, что области $\Omega^{ \pm}$назьваются липиицевыми, или имеющими липиицеву границу, если локально граница Г представляет собой график функции, удовлетворяюшей условию Липшица. После подходящего поворота системы координат такая функция имеет вид

$$
x_{n}=\varphi\left(x^{\prime}\right), \quad x^{\prime}=\left(x_{1}, \ldots, x_{n-1}\right) \in G, \quad\left|\varphi\left(x^{\prime}\right)-\varphi\left(y^{\prime}\right)\right| \leqslant C\left|x^{\prime}-y^{\prime}\right|,
$$

где $G$ - область в $\mathbb{R}^{n-1}$. Мы должны будем обстоятельно остановиться на исследованиях по разрешимости основных граничных задач и гладкости их решений в липшицевых областях. Основным в этой статье является традиционный вариационный подход с определением решения интегральным соотношением, но с дополнительным исследованием гладкости решений. Наряду с этим подходом в главе II в случае систем с постоянными коэффициентами используются поверхностные потенциалы на основе относительно недавно доказанных непростых теорем. Эти теоремы позволяют обойтись без (отсутствующего в этом случае) понятия эллиптического ПДО на границе и частично усилить результаты, получаемые при помоши вариационного подхода.

Возможности, предоставляемые вариационным методом и методом потенщиалов, сравниваются в $\S 5$ (ср. [28]).

В указанных вьше примерах спектральных задач, если значение фиксированного параметра вешественно, эти задачи приводятся к уравнениям с самосопряженными операторами соответственно в $L_{2}(\Omega)$ (первая задача) и в $L_{2}(\Gamma)$ (вторая задача). Самосопряженные задачи будут на первом плане в нашем обсуждении. Но мы затронем также несамосопряженные задачи, близкие к самосопряженньм (в $\S 3-6)$.

Мы также остановимся на спектральных задачах для уравнений типа (1) с кулоновскими особенностями в потенциале.

$\mathrm{Bce,} \mathrm{что} \mathrm{делал} \mathrm{автор} \mathrm{в} \mathrm{этих} \mathrm{направлениях,} \mathrm{стимулировалось} \mathrm{задачами,} \mathrm{о} \mathrm{которых}$ он узнал от физиков. Задачу второго типа для уравнения Гельмгольца и аналогичные внешние задачи и задачи сопряжения (тоже для уравнения Гельмгольца) поставили как задачи в теории дифракции Б.З. Каценеленбаум, Н. Н. Войтович и А. Н. Сивов (см. [126], а также [14]). Они же предложили рассматривать аналогичные задачи для системы Максвелла (имеющей первый порядок), которые мы кратко рассмотрим в п. 6.1. Последние не эллиптичны, но приводятся к эллиптическим уравнениям на гранище, если граница гладкая. Все эти задачи не всегда являются самосопряженными, но тогда они близки, а в некоторых интересных случаях “бесконечно близки" к самосопряженньм задачам. Отметим, что предложенные в [126] спектральные задачи вьглядели для физиков необычными из-за нетрадиционного выбора спектрального параметра (вместо частоты).

От польского физика Р. Шмытковского автор сравнительно недавно узнал о спектральных задачах для уравнения Шрёдингера, связанных с применяемьм в физике 
“методом $R$-матрищы”; в этих задачах потенциал может иметь кулоновские особенности [119]. Эти задачи приводят к обсуждаемому в нашей статье вопросу о том, в каких соболевских пространствах $H^{t}(\Omega)$ или $H^{t}(\Gamma)$ с положительнылм (точнее, ненулевым) $t$ собственные функции сохраняют базисность. Мы объясним понятие $R$-матрицы и связанные с ним вопросы в следующем пункте. Аналогичные вопросы возникают для системы Дирака [119], и мы кратко рассмотрим в п. 6.2 некоторые спектральные задачи для этой системы первого порядка.

Во всех задачах, которые мы будем рассматривать, включая задачи в неограниченной области $\Omega^{-}$и задачи сопряжения в $\Omega^{+} \cup \Omega^{-}$со спектральным параметром на $\Gamma$, спектр оказьвается состоящим из изолированных собственных значений конечной кратности и, возможно, нуля; собственные значения могут скапливаться только к бесконечности и/или к нулю. Две точки накопления, нуль и бесконечность, обнаруживаются у задач для системы Максвелла и для системы Дирака со спектральным параметром на $\Gamma$.

В отношении негладких границ следует отметить, что имеется обширная литература по эллиптическим задачам в областях с ребрами, коническими точками и т. п. (пример - внутренность куба), см., в частности, работу [62] и книги [67], [68] и [99]. В таких областях удается исследовать асимптотическое поведение решений вблизи особенностей границы. Для удобства мы будем (не вполне правильно!) называть такие границы стратифицированными. В физических задачах именно такие негладкие границы наиболее интересны, но области с разной геометрией особенностей границы приходится рассматривать раздельно. Мы предпочитаем сразу считать границу липшицевой, как делается в очень многих работах. (Не все области со стратифицированной границей липшицевы, известньй пример - два кирпича, верхний лежит на нижнем поперек нижнего.) Промежуточным является случай почти гладкой границы (это понятие введено в [11]) - поверхности, бесконечно гладкой вне замкнутого подмножества нулевой лебеговой меры. Мы иногда будем упоминать этот случай, включающий в себя все случаи стратифицированной границы, но без асимптотического анализа решений вблизи ее особенностей. Мы также кратко остановимся на относительно благоприятном случае уравнения Шрёдингера в выпуклой области. Вьпуклая область, как известно, всегда липшищева.

Изложение поневоле является сжатым, во многих местах приходится ограничиться формулировками и схематическим объяснением доказательств со ссылками на литературу. Но в тех случаях, когда обсуждаются новые вопросы, доказательства проводятся подробно.

Отметим также, что мы не пытаемся рассмотреть все вопросы в единой общности и в некоторых ситуациях вводим упрощаюшие предположения.

Все необходимые определения даются и технические средства объясняются по мере надобности. Поэтому до чтения главы II следует просмотреть главу I.

Наиболее сушественные, на наш взгляд, вопросы, остающиеся пока нерешенными, сформулированы в п. 7.2.

Все результаты автора, включенные в эту работу, рассказывались и обсуждались на университетском семинаре Марка Иосифовича Вишика. В связи с его 80-летним юбилеем хочу пожелать ему здоровья, благополучия и многих новых замечательных достижений в математике. 
2. $R$-матрица. Вернемся к задаче (1), (2), но граничное условие предположим неоднородным:

$$
\partial_{\nu} u^{+}(x)+\mu u^{+}(x)=g(x) .
$$

Пусть вещественные параметры $\lambda$ и $\mu$ выбраны и зафиксированы так, что задача $(1),(4)$ однозначно разрешима (при любой $g$; пока мы не обсуждаем выбор соответствующих функциональных пространств). Тогда определен оператор

$$
\mathscr{R}: g \mapsto u^{+},
$$

которьй специалисты по атомной и молекулярной физике назьвают $R$-матрицей. ${ }^{1}$ Это чрезвычайно популярное понятие, используемое в огромном числе физических работ (см., в частности, [119], обзор [23] и приведенную там литературу). Одними из первых были работы Вигнера (1946 г.). Одна или несколько частиц окружаются поверхностью $\Gamma$, и информация о том, что происходит внутри области $\Omega$ с этой границей, “считывается” по наблюдениям на Г.

Для построения $R$-матрицы физики используют как первую, так и вторую спектральную задачу, с которых мы начали п. 1 [119]. Пусть сначала в качестве спектрального параметра взято $\lambda$. Соответствуюшая спектральная задача (с $g=0)-$ формально самосопряженная (слово “формально” мы объясним в п. 1.1), и сушествует ортонормированньй базис в $L_{2}(\Omega)$, состояший из ее собственных функщий $u_{l}(x)$ $(l=1,2, \ldots)$; пусть $\lambda_{l}$ - соответствуюшие собственные значения. Тогда, в частности, для решения неоднородной задачи (1), (4) мы имеем

$$
u(x)=\sum_{1}^{\infty} c_{l} u_{l}(x), \quad c_{l}=\left(u, u_{l}\right)_{\Omega},
$$

где $(\cdot, \cdot)_{\Omega}$ - стандартное скалярное произведение в $H^{0}(\Omega)=L_{2}(\Omega)$. Допустим, что мы знаем собственные функции и собственные значения; как тогда вычислить коэффициенты Фурье $c_{l}$ ? Для этого надо проинтегрировать по частям два раза выражение $\left(\Delta u, u_{l}\right)_{\Omega}$. Мы получим

$$
\left(\Delta u, u_{l}\right)_{\Omega}=\left(\partial_{\nu} u^{+}, u_{l}^{+}\right)_{\Gamma}-\left(u^{+}, \partial_{\nu} u_{l}^{+}\right)_{\Gamma}+\left(u, \Delta u_{l}\right)_{\Omega},
$$

где $(\cdot, \cdot)_{\Gamma}$ - стандартное скалярное произведение в $H^{0}(\Gamma)=L_{2}(\Gamma)$. Используя теперь уравнения и граничные условия для $u$ и $u_{l}$, получаем желаемое выражение для $c_{l}$ и приходим к формуле

$$
u(x)=\sum_{1}^{\infty} \frac{\left(g, u_{l}^{+}\right)_{\Gamma}}{\lambda_{l}-\lambda} u_{l}(x) .
$$

Это несколько парадоксальная формула: решение задачи с неоднородным граничньм условием разложено в ряд по собственным функциям, удовлетворяющим одному и тому же однородному граничному условию. Тем не менее, именно она наиболее популярна у физиков. Ряд (7) заведомо сходится в $H^{0}(\Omega)$. Формально переходя в нем к граничным значениям, получаем

$$
u^{+}(x)=\sum_{1}^{\infty} \frac{\left(g, u_{l}^{+}\right) \Gamma}{\lambda_{l}-\lambda} u_{l}^{+}(x) .
$$

\footnotetext{
${ }^{1}$ В квантовом методе для обратной задачи рассеяния есть другое понятие $R$-матрицы; между этими понятиями нет ничего общего.
} 
Это первая формула для $R$-матрищь. Можно ли пользоваться этим разложением? В 1974 г. Наркович показал [96], что при $g \in H^{0}(\Omega)$ оно справедливо в $H^{0}(\Gamma)$. Других работ с математическим исследованием спектральных подходов к построению $R$-матрицы, по-видимому, не было. Как мы увидим в главе I, этот результат из [96] усиливается: в (7) имеет место сходимость в соболевском пространстве $H^{t}(\Omega)$ при $0 \leqslant t<3 / 2$, и как следствие разложение (8) сходится в $H^{t}(\Gamma)$ при $0 \leqslant t<1$.

Здесь вообще возникает вопрос, в каких соболевских пространствах $H^{t}(\Omega), t \geqslant 0$, собственные функии $u_{l}$ самосопряженной задачи для эллиптической системы второго порядка (с однородньм граничным условием на ее границе) сохраняют свойства базисности. В случае гладкой границы сравнительно легко показать, что это пространства $H^{t}(\Omega)$ именно с $t<3 / 2$ (ср. [5], [6] и приведенную там литературу). Но интересно, что этот же ответ сохраняется в случае липиицевой границь (более полньй результат для $\pm t>0$ см. в $\S 2)$. По-видимому, последний результат не отмечался раньше в литературе. Для его доказательства мы обобщаем теорему Саваре [111] о гладкости обобшенных решений в лишшицевых областях.

Указанная спектральная задача представляет интерес для физиков в случае, когда потенциал $V(x)$ в (1) содержит кулоновские особенности. В простейшем случае, когда рассматривается одна частица в центральном поле, это слагаемое вида $c /|x|$, при этом $n=3$ и границу можно считать гладкой. В случае нескольких частиц $n=3 k$ и сингулярная часть потенциала состоит из слагаемых вида $c_{\alpha} /\left|x^{\alpha}\right|$ и с $\alpha \beta /\left|x^{\alpha}-x^{\beta}\right|$, где подразумевается, что набор координат $\left(x_{1}, \ldots, x_{n}\right)$ разбит на трехмерные группы $x^{1}, \ldots, x^{k}$. Область $\Omega$ берется в виде $\Omega_{0} \times \cdots \times \Omega_{0}(k$ сомножителей $)$, где $\Omega_{0}-$ трехмерная область с гладкой границей. Очевидно, что гранища такой области $\Omega$ при $k>1$ будет негладкой. Ее можно сгладить, но, по-видимому, это усложняет расчеты. В [96] допускается вхождение в потенциал кулоновских слагаемых, но граница считается гладкой. Бегло мы затронем также более глубокие особенности (в п. 3.4).

Добавим, что в случае гладкой гранищы имеется стандартная возможность перевода неоднородности из граничного условия в систему, по-видимому не используемая физиками. Как мы увидим в п. 1.4, она позволяет прибавить к правой части в (8) некоторый ряд по тем же функциям $\varphi_{l}$ с нулевой суммой, улучшая сходимость: например, при $g \in H^{1 / 2}(\Gamma)$ она будет иметь место в $H^{3 / 2}(\Gamma)$; при $n=3$ отсюда следует равномерная сходимость. Но в случае липшицевой гранищы такой возможности, вообще говоря, нет.

Второй подход к построению $R$-матрицы состоит по существу в сведе́нии задачи (1), (4) к уравнению вида

$$
\mathscr{T} \varphi=\mu \varphi-g
$$

на $Г$, где $\varphi=u^{+}$и $\mathscr{T}$ - хорошо известный в литературе оператор, переводяший данные Дирихле в данные Неймана с изменением знака (последнее будет удобнее в главе II):

$$
\mathscr{T} u^{+}=-\partial_{\nu} u^{+}
$$

(Оператор - $\mathscr{T}$ назьвают также оператором Пуанкаре-Стеклова.) Дополнительные условия, при которых этот оператор существует и обратим, мы уточним в главе II. По существу они состоят в том, что при данном $\lambda$ для задач Дирихле и Неймана имеет место однозначная разрешимость. Этот оператор оказьвается самосопряженным 
в $L_{2}(\Gamma)$, и если мы знаем ортонормированньй базис $\left\{\varphi_{l}\right\}_{1}^{\infty}$ из его собственных функций и соответствуюшие собственные значения $\mu_{l}$, то граничное значение решения задачи (1), (4) строится по формуле

$$
u^{+}(x)=\sum_{1}^{\infty} \frac{\left(g, \varphi_{l}\right)_{\Gamma}}{\mu-\mu_{l}} \varphi_{l}(x) .
$$

Это вторая формула для $R$-матрицы, мы обсудим ее в главе II. В случае гладкой гранищы $\mathscr{T}$ - эллиптический ПДО первого порядка и характер сходимости здесь неограниченно улучшается с ростом гладкости функции $g$. В случае липшицевой границь при $g \in H^{0}(\Gamma)$ удается установить сходимость ряда (11) в $H^{t}(\Gamma)$ при $|t|<1$ и для некоторых особенно интересных уравнений и систем также при $t= \pm 1$. Перейти через эти точки нельзя, так как пространства $H^{t}(\Gamma)$ определяются на липшицевой поверхности в общем случае только при $|t| \leqslant 1$.

3. Некоторые обозначения. Через $C_{j}$ в дальнейшем обозначаются различные положительные постоянные. Обычно мы их будем нумеровать заново в каждом новом параграфе, а иногда и в новом пункте. Через $\|\cdot\|_{r, \Omega}$ и $\|\cdot\|_{r, \Gamma}$ обозначаются нормы в пространствах $H^{r}(\Omega)$ и $H^{r}(\Gamma)$. Размерности вектор-функций из этих пространств в наших обозначениях явно не указьваются, но будут ясны из контекста.

Через $O_{R}(x)$ обозначается открытый шар радиуса $R$ в $\mathbb{R}^{n}$ с центром в точке $x$. Если $x$ - начало координат, то пишем просто $O_{R}$.

Пусть положительное число $R$ настолько велико, что замыкание $\bar{\Omega}$ области $\Omega$ лежит внутри $O_{R}$. Тогда пересечение $\Omega^{-} \cap O_{R}$ обозначается через $\Omega_{R}^{-}$.

Через $I$ обозначаются единичньй оператор и единичная матрица.

В ссылках на книги и другие работы “т." - теорема, “п." - пункт, “пр." - предложение, "сл." - следствие.

\section{Глава I. Задачи со спектральным параметром в системе}

\section{§1. Задачи в области с гладкой границей}

1.1. Класс рассматриваемых систем и граничные условия. Теоремы об однозначной разрешимости граничных задач. Системы, рассматриваемые в настоящей статье, будут иметь вид

$$
L u(x)=\lambda u(x)+f(x), \quad \text { где } L u(x):=\left[L_{0}+\sum b_{j}(x) \partial_{j} u(x)+c(x)\right] u(x) .
$$

Здесь $u(x)$ - комплекснозначная вектор-функция, столбец $\left(u_{1}(x), \ldots, u_{m}(x)\right)^{\prime}$ высоты $m$, и $\partial_{j}-$ частные производные $\partial / \partial x_{j}$. Главная часть $L_{0} u(x)$ этой системы записьвается в виде

$$
L_{0} u(x):=-\sum \partial_{j}\left[a_{j k}(x) \partial_{k} u(x)\right]
$$

В этой статье мы всюду предполагаем, что элементы $a_{j k}^{r s}(x)$ матрицы $a_{j k}(x)=\left(a_{j k}^{r s}(x)\right)$ подчинены условию симметрии

$$
a_{j k}^{*}(x)=a_{k j}(x), \quad \text { т.е. } a_{j k}^{r s}(x)=\overline{a_{k j}^{s r}(x)}
$$


Граничное условие, представляюшее для нас наибольший интерес, имеет вид

$$
B u:=\partial_{\nu_{a}} u^{+}(x)+b(x) u^{+}(x)=g(x) .
$$

Здесь $\partial_{\nu_{a}}-$ конормальная производная в точках $x$ границы:

$$
\partial_{\nu_{a}} u^{+}(x)=\sum \nu_{j}(x) a_{j k}(x) \partial_{k} u^{+}(x)
$$

Выделять в (1.4) слагаемое с параметром $\mu$ пока нет необходимости. При $b(x)=0$ будем назьвать эту задачу задачей Неймана. Мы рассмотрим также задачу Дирихле с граничным условием Дирихле

$$
u^{+}(x)=h(x)
$$

но в этой первой главе она не будет на первом плане. Матрицы $a_{j k}(x), b_{j}(x)$ и с $(x)$ в настояшем и следуюшем пунктах предполагаются бесконечно гладкими в замыкании $\bar{\Omega}$ области $\Omega$, а матрица $b(x)$ - бесконечно гладкой на Г. Областью, как обычно, мы называем открытое связное множество.

Приведем теперь дополнительные предположения, приводящие к самосопряженности задачи. Проще всего предположить, что матрицы $b_{j}(x)$ нулевые, а матрица $c(x)$ эрмитова: $c^{*}(x)=c(x)$. При этих предположениях, как показывает двукратное интегрирование по частям, на функциях $u, v$ из $C_{0}^{\infty}(\Omega)$

$$
(L u, v)_{\Omega}=(u, L v)_{\Omega}
$$

Оператор $L$, для которого верно такое соотношение, назьвается формально самосопря жсенным, и система (1.1) (с $\lambda=0$ или вещественным $\lambda$ ) тоже назьвается формально самосопряженной. Соотношение (1.7) сохраняется для функций $u, v$ из $H^{2}(\Omega)$, удовлетворяюших однородным условиям Дирихле $u^{+}=v^{+}=0$, что позволяет назвать задачу Дирихле формально самосопряженной. Для функций, удовлетворяюших однородному граничному условию вида (1.4), соотношение (1.7) тоже сохраняется, если матрица $b(x)$ эрмитова. В этом случае скажем, что задача $(1.1),(1.4)$ является формально самосопряженной. В частности, это относится к задаче Неймана с $b=0$.

Более общие условия, при которых оператор $L$ и задача (1.1), (1.4) являются формально самосопряженньми, несколько более громоздки и состоят в следующем:

$$
b_{j}^{*}=-b_{j}, \quad c^{*}=c-\sum \partial_{j} b_{j} \quad \text { в } \Omega, \quad b^{*}=b-\sum \nu_{j} b_{j} \text { на } \Gamma .
$$

Если задача (1.1), (1.4) не является формально самосопряженной, то строится формально сопряженная к ней задача с операторами $L^{(*)}, B^{(*)}$ вместо $L, B$. Вместо (1.7) тогда имеет место формула

$$
(L u, v)_{\Omega}=\left(u, L^{(*)} v\right)_{\Omega}
$$

для функций $u, v$ из $H^{2}(\Omega)$, удовлетворяющих соответственно однородным граничньм условиям $B u=0$ и $B^{(*)} v=0$. Операторы $L^{(*)}$ и $B^{(*)}$ легко вьписываются. Они имеют структуру, аналогичную структуре операторов $L$ и $B$, и отличаются от последних только младшими членами.

Теоремы об однозначной разрешимости задач и гладкости их решений мы будем обсуждать без предположений о формальной самосопряженности. 
Матрица

$$
a(x, \xi)=\sum a_{j k}(x) \xi_{j} \xi_{k}
$$

называется главным символом оператора $L .^{2}$ Из (1.3) следует, что она эрмитова. Система (1.1) назьвается сильно эллиптической, если $a(x, \xi)$ - положительно определенная матрица равномерно при $x \in \bar{\Omega}$ и/или $x \in \overline{\Omega^{-}}$и вешественных $\xi$ с $|\xi|=1$. Условие сильной эллиптичности в дальнейшем предполагается вьполненным и для удобства назьвается условием I. Из него следует эллиптичность системы $(\operatorname{det} a(x, \xi) \neq 0)$, ее правильная эллиптичность и эллиптичность задачи Дирихле, т.е. для нее выполнено условие Шапиро-Лопатинского. Более того, хорошо известен следуюший результат (см., например, [103] или [83; гл. 4]).

Теорема 1.1. Пусть выполнено условие I. Тогда существует такое $\lambda_{0} \in \mathbb{R}$, что задача Дирихле (1.1), (1.6) $c \operatorname{Re} \lambda<\lambda_{0}$ имеет одно и только одно решение в $H^{2}(\Omega)$ при любых $f \in H^{0}(\Omega)$ и $h \in H^{3 / 2}(\Gamma)$, причем соответствуюшее отображсение $u \mapsto(f, h)$ определяет изоморфизм (непрерывный в обе стороны) пространства $H^{2}(\Omega)$ на прямое произведение пространств $H^{0}(\Omega)$ и $H^{3 / 2}(\Gamma)$. Eсли $f \in C^{\infty}(\bar{\Omega})$ и $h \in C^{\infty}(\Gamma)$, то решение принадлежит $C^{\infty}(\bar{\Omega})$.

Несколько сложнее обстоит дело с задачей (1.1), (1.4). Чтобы объяснить разницу, введем билинейную, точнее полуторалинейную, форму $\Phi(u, v)$, отвечающую этой задаче. Однократным интегрированием по частям с последуюшим использованием граничного условия получаем для решения $u$ уравнения (1.1) из $H^{2}(\Omega)$ и любой функции $v$ из $H^{1}(\Omega)$ соотношение

$$
(f, v)_{\Omega}+\lambda(u, v)_{\Omega}=(L u, v)_{\Omega}=\Phi(u, v)-\left(g, v^{+}\right)_{\Gamma}
$$

где

$$
\begin{gathered}
\Phi(u, v)=\Phi_{0}(u, v)+\sum\left(b_{j} \partial_{j} u, v\right)_{\Omega}+(c u, v)_{\Omega}+\left(b u^{+}, v^{+}\right)_{\Gamma}, \\
\Phi_{0}(u, v)=\int_{\Omega} E(u, v) d x \text { и } E(u, v)=\sum a_{j k}^{r s} \partial_{k} u_{s} \partial_{j} \bar{v}_{r} .
\end{gathered}
$$

Форма $\Phi_{0}(u, v)$ эрмитова, т.е. совпадает с $\overline{\Phi_{0}(v, u)}$, и является главной частью формы $\Phi(u, v)$. Из сильной эллиптичности вьводится неравенство Гординга

$$
\Phi_{0}(u, u) \geqslant C_{1}\|u\|_{1, \Omega}^{2}-C_{2}\|u\|_{0, \Omega}^{2}
$$

на функциях из $H_{0}^{1}(\Omega)$ - подпространства функций в $H^{1}(\Omega)$, удовлетворяющих однородному условию Дирихле (см., например, [83]). Говорят, что форма $\Phi_{0}(u, v)$ коэриитивна на этом подпространстве. Это ключевой момент в исследовании задачи Дирихле для сильно эллиптической системы. Как следствие отвечающая задаче Дирихле форма $\Phi(u, v)$ (без интеграла по $\Gamma)$ тоже коэрцитивна на $H_{0}^{1}(\Omega)$ в смысле

$$
\operatorname{Re} \Phi(u, u) \geqslant C_{3}\|u\|_{1, \Omega}^{2}-C_{4}\|u\|_{0, \Omega}^{2}
$$

\footnotetext{
2Здесь знак справа объясняется следующим образом: $L_{0} u(x)=\sum D_{j} a_{j k}(x) D_{k} u(x)$, где $D_{j}=-i \partial_{j}$ переходит в умножение на $\xi_{j}$ при преобразовании Фурье.
} 
на функциях из этого подпространства (ниже мы проверяем похожее предложение 1.3).

В случае задачи Неймана или, более общо, задачи с граничным условием (1.4) нужна коэрцитивность формы $\Phi_{0}(u, v)$ на $H^{1}(\Omega)$, т.е. справедливость неравенства вида (1.14) на этом пространстве. Сильной эллиптичности для этого недостаточно (см. пример 5 в следующем пункте), и мы дополним условие I следуюшим условием в точках границы.

УСловИе II. $\sum a_{j k}^{r s}(x) \zeta_{k}^{s} \overline{\zeta_{j}^{r}} \geqslant C_{5} \sum\left|\zeta_{j}^{r}+\zeta_{r}^{j}\right|^{2}$ nрu $x \in \Gamma$ икомплексных $\zeta_{j}^{r}$.

Здесь подразумевается, что при $m \neq n$ "недостаюшие" $\zeta_{r}^{j}($ с $j>m$ или $r>n)$ заменяются нулями. Более просто вьглядит

УСловиЕ III. $\sum a_{j k}^{r s}(x) \zeta_{k}^{s} \overline{\zeta_{j}^{r}} \geqslant C_{6} \sum\left|\zeta_{j}^{r}\right|^{2}$ nри $x \in \Gamma$ икомплексных $\zeta_{j}^{r}$.

Если коэффищиенты $a_{j k}^{r s}$ постоянны, то нет нужды различать условия при $x \in \Gamma$ и $x \in \overline{\Omega^{ \pm}}$, и в этом случае договоримся считать, что условия II и III относятся ко всем $x$. Как легко проверить, в этом случае $\mathrm{I} \Leftarrow \mathrm{II} \Leftarrow \mathrm{III}$. Условие III в матричном случае $(m>1)$ очень жесткое и потому нежелательное.

Но в скалярном случае $(m=1)$ дело обстоит следуюшим образом. Условие (1.3) при гладких $a_{j k}(x)$ позволяет предположить, что все $a_{j k}(x)$ вешественны. Тогда условие I эквивалентно условию положительной определенности матрицы $\left(a_{j k}(x)\right)$ и, значит, эквивалентно условию III при всех $x \in \overline{\Omega^{ \pm}}$. Однако следует иметь в виду, что конормальная производная, вообще говоря, меняется при переходе от комплексных коэффициентов к вешественным. См. пример 5 в следуюшем пункте.

Все три условия влекут те же условия для формально сопряженной системы, так как не затрагивают младшие члены.

Теперь нам понадобится второе неравенство Корна

$$
\int_{\Omega} \sum\left|\partial_{j} u^{r}(x)+\partial_{r} u^{j}(x)\right|^{2} d x \geqslant C_{7}\|u\|_{1, \Omega}^{2}-C_{8}\|u\|_{0, \Omega}^{2}, \quad u \in H^{1}(\Omega) .
$$

Это неравенство для вещественнозначных функций из $H^{1}(\Omega)$ хорошо известно при $m=n$ (см., например, $[64 ; \S 2]$ или $[104 ; \S 2]){ }^{3}$ Как следствие оно верно, во-первых, для комплекснозначных функций и, во-вторых, при любых $m$ и $n$.

ПРЕДЛОЖЕНИЕ 1.2. При условиях I $и$ II форма $\Phi_{0}(u, v)$ коэрцитивна на $H^{1}(\Omega)$.

ДоКАЗАТЕЛЬСТВо проводится очевидньм образом при помощи разбиения единицы в $\bar{\Omega}$ и второго неравенства Корна с учетом того, что условие II остается справедливым в некоторой граничной полоске.

ПРЕДЛОЖЕНИЕ 1.3. При тех жсе условиях форма $\Phi(u, v)$ коэриитивна на $H^{1}(\Omega)$.

Мы проведем несложное доказательство (хотя можно было бы сослаться, например, на [83]), это повод для того, чтобы выписать оценки младших членов; эти оценки понадобятся и дальше.

\footnotetext{
${ }^{3}$ Первым нерав енством Корна называют аналогичное неравенство на функциях из $H_{0}^{1}(\Omega)$.
} 
ДокАЗАТЕЛЬСтво. Ясно, что

$$
\begin{gathered}
\left|\left(b_{j} \partial_{j} u, u\right)_{\Omega}\right| \leqslant C_{9}\|u\|_{1, \Omega}\|u\|_{0, \Omega}, \\
\left|(c u, u)_{\Omega}\right| \leqslant C_{10}\|u\|_{0, \Omega}^{2} .
\end{gathered}
$$

Далее,

$$
\left|\left(b u^{+}, u^{+}\right)_{\Gamma}\right| \leqslant C_{11}\left\|u^{+}\right\|_{0, \Gamma}^{2} \leqslant C_{12}\|u\|_{1, \Omega}\|u\|_{0, \Omega},
$$

cp. [54]. Последнее неравенство вытекает из неравенства

$$
q\|u\|_{0, \Gamma}^{2} \leqslant C_{13}\left(\|u\|_{1, \Omega}^{2}+q^{2}\|u\|_{0, \Omega}^{2}\right),
$$

где постоянная не зависит от $q>0$ : достаточно поделить его на $q$ и положить $q=$ $\|u\|_{1, \Omega} /\|u\|_{0, \Omega}$. Последнее неравенство в случае полупространства вьведено при помощи преобразования Фурье в [16; $§ 1]$.

Из оценок (1.17)-(1.19) следует, что сумма младших членов в форме $\Phi$ не превосходит $\varepsilon\|u\|_{1, \Omega}^{2}+C_{\varepsilon}\|u\|_{0, \Omega}^{2}$ со сколь угодно малым положительным $\varepsilon$, так что на функциях из $H^{1}(\Omega)$ получается неравенство вида (1.15).

ПРЕДЛОЖЕНИЕ 1.4. При условияХ I и II задача (1.1), (1.4) әллиптична.

ДокАЗАТЕЛЬСТво сводится к проверке условия Шапиро-Лопатинского для системы (1.1) с постоянными коэффициентами без младших членов и для граничного условия Неймана в полупространстве при условии II. В случае вещественных $a_{j k}^{r s}$ проверка проведена в [9; пр. 2.4]. В общем случае проходит аналогичное рассуждение.

Условие II существенно: см. пример 5 в следующем пункте.

Теорема 1.5. Пусть выполнены условия I $и$ II. Тогда существует такое $\lambda_{0}$, что задача (1.1), (1.4) имеет при $\operatorname{Re} \lambda<\lambda_{0}$ одно и только одно решение в $H^{1}(\Omega)$ для любых $f \in H^{0}(\Omega)$ и $g \in H^{1 / 2}(\Gamma)$. При этом отображение $u \mapsto(f, g)$ определяет изоморфизм пространства $H^{2}(\Omega)$ и прямого произведения пространств $H^{0}(\Omega)$ и $H^{1 / 2}(\Gamma)$. При $f \in C^{\infty}(\bar{\Omega}), g \in C^{\infty}(\Gamma)$ решение принадлежит $C^{\infty}(\bar{\Omega})$.

ДоКАЗАТЕЛЬСТво. В силу эллиптичности задачи отображение $u \mapsto(f, g)$ фредгольмово. Из предложения 1.3 вьводится единственность как для данной, так и для формально сопряженной задачи при $\operatorname{Re} \lambda<\lambda_{0}$. Значит, индекс задачи нулевой и задача однозначно разрешима при этих $\lambda$. Далее используется известная теорема о гладкости решений эллиптических задач.

1.2. Примеры систем. В приведенных дальше примерах 1-4 изменен знак левой части.

ПримеР 1. Уравнение Гельмгольца

$$
\Delta u(x)+\omega^{2} u(x)=f(x) .
$$

В этом случае $m=1$ и $\partial_{\nu_{a}}=\partial_{\nu}$ - обычная нормальная производная. Более общий пример: система разделенных уравнений Гельмгольца $(m>1)$ с общим или разными $\omega^{2}$. Вьполнено условие III (при всех $x$ ). 
Пример 2. Скалярное ( $m=1)$ уравнение Шрёдингера с переменными, вообще говоря, старшими коэффициентами:

$$
\operatorname{div} A(x) \operatorname{grad} u(x)-V(x) u(x)=f(x),
$$

где $A(x)$ - вешественная симметрическая положительно определенная матрица и $V(x)$ - вешественная функция. Вьполнено условие III (при всех $x$ ). При желании читатель может ограничиться разбором дальнейшего материала настояшей главы на этом примере.

ПримеР 3. Система Ламе

$$
\mu_{0} \Delta u(x)+\left(\lambda_{0}+\mu_{0}\right) \operatorname{grad} \operatorname{div} u(x)+\omega^{2} u(x)=f(x) .
$$

Здесь $m=n ; \lambda_{0}$ и $\mu_{0}-$ коэффициенты Ламе (для простоты считаем их постоянными), подчиненные неравенствам $\mu_{0}>0, n \mu_{0}+2 \mu_{0}>0$. Отсюда следует, что вьполнено условие II (при всех $x$ ), условие III не вьполнено. Это частньй случай следующего примера.

ПримеР 4. Обобшенная система анизотропной упругости. Она имеет вид

$$
-L_{0} u(x)+\omega^{2} u(x)=f(x),
$$

где $m=n$ и коэффищиенты старшей части вешественны и подчинены дополнительному условию симметрии $a_{j k}^{r s}(x)=a_{r k}^{j s}(x)$. Предполагается, что условие III вьполнено для вешественных симметричных матриц $\left(\zeta_{j}^{r}\right)$ при всех $x \in \bar{\Omega}$. Отсюда следует, что вьполнено условие II при всех $x$ (см., например, [104; 33$]$ ).

ПримеР 5. Как хорошо известно, конормальная производная зависит от способа записи оператора $L_{0}$ в виде (1.2) (см., например, [32]). Покажем на простом примере, что, меняя способ записи, можно потерять и условие II, и эллиптичность задачи Неймана.

Запишем оператор Лапласа в виде $\partial_{1}^{2}+\partial_{2}^{2}+i \partial_{1} \partial_{2}-i \partial_{2} \partial_{1}$. Соответствуюшая форма в условии II или III имеет вид $\left|\zeta_{1}\right|^{2}+\left|\zeta_{2}\right|^{2}+i \zeta_{2} \bar{\zeta}_{1}-i \zeta_{1} \bar{\zeta}_{2}$. Как легко видеть, она обрашается в 0 при $\zeta_{2}=i, \zeta_{1}=1$. Конормальная производная имеет вид $\nu_{1} \partial_{1}+\nu_{2} \partial_{2}+i \nu_{1} \partial_{2}-i \nu_{2} \partial_{1}$. Легко проверить, что отвечающая ей задача Неймана в единичном круге не удовлетворяет условию Шапиро-Лопатинского в точке $(1,0)$ : уравнение $\left(\partial^{2}-\xi^{2}\right) v(t)=0$ на луче $t<0$ с граничным условием $(\partial+\xi) v(0)=0$ имеет нетривиальные убьваюшие при $t \rightarrow-\infty$ решения для $\xi<0$.

\section{3. Самосопряженная спектральная задача в случае бесконечной глад-} кости границы и коэффициентов. В этом пункте мы определим оператор $\mathscr{L}$, отвечающий такой задаче с однородным граничным условием (1.4) (где $g=0$ ), и обсудим спектральные свойства этого оператора. Напомним, что мы предполагаем вьполненными условия I, II, (1.3) и условия формальной самосопряженности (см. п. 1.1). Обозначим через $H^{2}(\Omega, B)$ подпространство в пространстве $H^{2}(\Omega)$, выделяемое граничным условием $B u=0$. 
Теперь определим неограниченный оператор $\mathscr{L}$ в $H^{0}(\Omega)$ с областью определения $H^{2}(\Omega, B)$, действуюший по формуле $\mathscr{L} u=L u$. Этот же оператор можно рассматривать как ограниченный оператор из $H^{2}(\Omega, B)$ в $H^{0}(\Omega)$, и в этом качестве он фредгольмов ввиду эллиптичности задачи. Из эллиптичности и формальной самосопряженности следует, что $\mathscr{L}$ - самосопряженньй оператор в $H^{0}(\Omega)$ с вещественным $\partial u c$ кретны.м спектром (т.е. этот спектр состоит из изолированных собственных значений конечной кратности). В силу предложения 1.3 оператор полуограничен снизу.

Для собственных значений $\lambda_{l}$ оператора $\mathscr{L}$, занумерованных в порядке неубывания с учетом кратностей, справедлива асимптотическая формула

$$
\lambda_{l}=C^{(n)} l^{2 / n}+O\left(l^{1 / n}\right)
$$

где положительную постоянную $C^{(n)}$ можно выразить через главньй символ $a(x, \xi)$, точнее, через функцию распределения его собственных значений (см. ниже формулу (2.43)). Здесь оценка остатка в обшем случае неулучшаема, ее для систем получил Иврий (см. [48]). По скалярному случаю $(m=1)$ имеется обстоятельно написанная книга Васильева и Сафарова [110]. Относительно предыстории этих результатов см. приведенные там указания.

Из соответствуюших собственных функщий $u_{l}(x)$ составляется ортонормированньй базис в $H^{0}(\Omega)$. Они принадлежат $C^{\infty}(\bar{\Omega})$. Нам осталось проверить следующее предложение. Напомним, что базис в банаховом пространстве называется безусловwblм, если он остается базисом после любой перестановки его членов.

ПРЕДЛОЖЕНИЕ 1.6. Система $\left\{u_{l}\right\}$ остается безусловным базисом в соболевских пространствах $H^{t}(\Omega)$ при $0<t<3 / 2$. При $3 / 2<t \leqslant 2$ это безусловный базис в подпространстве $H^{t}(\Omega, B)$ пространства $H^{t}(\Omega)$, выделяемом однородным м граничным условием Ви $=0$.

ДокаЗАТЕльство. Воспользуемся результатами Гривара [43] - Сили [116] об интерполящии подпространств соболевских пространств с граничными условиями. Обозначим через $\widetilde{H}^{t}(\Omega, B), 0<t<2$, интерполящионное пространство

$$
\left[H^{0}(\Omega), H^{2}(\Omega, B)\right]_{t / 2}
$$

(Здесь имеется в виду комплексная интерполяция, определения см., например, в [17].) При $3 / 2<t<2$ оно совпадает с $H^{t}(\Omega, B)$ - подпространством в $H^{t}(\Omega)$, выделяемьм граничным условием $B u=0$. При $t=3 / 2$ это незамкнутьй линеал в $H^{t}(\Omega)$ и граничное условие сохраняется в некотором слабом смысле. А при $0 \leqslant t<3 / 2$ мы имеем $\widetilde{H}^{t}(\Omega, B)=H^{t}(\Omega)$. Сдвигая, если нужно, спектральньй параметр, мы можем предположить, что все собственные значения $\lambda_{l}$ оператора $\mathscr{L}$ положительны. Тогда легко определяются степени этого оператора, и оператор $\mathscr{L}^{t / 2}$ устанавливает изоморфизм между $\widetilde{H}^{t}(\Omega, B)$ и $H^{0}(\Omega)$. (Здесь мы пользуемся самосопряженностью оператора $\mathscr{L}$, см. [73].) При $0<t<3 / 2$ это изоморфизм между $H^{t}(\Omega)$ и $H^{0}(\Omega)$. Оператор $\mathscr{L}^{-t / 2}$ переводит функции $u_{l}$ в себя с точностью до множителей $\lambda_{l}^{-t / 2}$, и получается безусловньй базис в $H^{t}(\Omega)$ при $0 \leqslant t<3 / 2$ и в $H^{t}(\Omega, B)$ при $3 / 2<t \leqslant 2$. Cp. [5], [6], где рассмотрены также бо́льшие $t$. 
Добавим, что этот базис в $H^{t}(\Omega)$ или $H^{t}(\Omega, B)$ станет ортогональным, если определить там скалярное произведение формулой $(u, v)_{t, \Omega}=\left(\mathscr{L}^{t / 2} u, \mathscr{L}^{t / 2} v\right)_{\Omega}$, и ортонормированным после соответствующей нормировки. Соответствующая норма эквивалентна обычной норме $\|\cdot\|_{t, \Omega}$. Это замечание можно сделать и к аналогичным формулировкам в дальнейших параграфах.

Все эти фактыпо сушеству известны или близки к известным. Для удобства ссылок мы объединим их в следующей теореме.

ТЕОРема 1.7. Пусть выполнены предположения, указанные в начале пункта, и пусть граница и все коэффициенты бесконечно гладкие. Тогда спектральной задаче $L u=\lambda u, B u=0$ отвечает оператор $\mathscr{L}$ в $H^{0}(\Omega)$ с дискретным спектром, являющийся самосопряжсенным и полуограниченным снизу. Его собственные значения $\lambda_{l}$ имеют асимптотику (1.25). Собственные функиии принадлежсат $C^{\infty}(\bar{\Omega})$, и составленный из них ортонормированный базис в $H^{0}(\Omega)$ остается безусловным базисом в $H^{t}(\Omega)$ при $0<t<3 / 2$ и в $H^{t}(\Omega, B)$ nри $3 / 2<t \leqslant 2$.

\section{4. Первая формула для $R$-матрицы.}

СлЕДСТВИЕ 1.8. При предположсениях теоремы 1.7 , если $f=0, g \in H^{0}(\Omega) u$ $\lambda$ отлично от собственных значений, ряд вида (8) сходится в $H^{t}(\Gamma)$ при $t<1$.

Здесь нужно пояснить только возможность взять $g$ в $H^{0}(\Gamma)$. При обычной в эллиптической теории постановке граничной задачи $g$ берется в $H^{1 / 2}(\Gamma)$ и решение принадлежит $H^{2}(\Omega)$. Однако при $f=0$ можно, используя двойственность и интерполяцию, понизить индексы, в частности, на 1/2, см., например, [74; гл. 2] и [115]. Здесь существенно, что система однородна. Решение $u$ принадлежит $H^{3 / 2}(\Omega)$, его разложение по собственньм функциям сходится к нему в $H^{t}(\Omega)$ при $t<3 / 2$, след $u^{+}$принадлежит $H^{1}(\Gamma)$, а его разложение по следам собственных функций сходится к нему в $H^{t}(\Gamma)$ при $t<1$. Можно и дальше понижать индексы, но это мы уже будем обсуждать в $\S 2$ для задач в липшицевых областях.

Теперь мы объясним, что дает перевод неоднородности из граничного условия в систему. Для простоты сделаем это на примере задачи $(1),(2)$ из введения, предполагая ее однозначно разрешимой. Пусть $g \in H^{1 / 2}(\Gamma)$ и $u_{0}$ - любая функция из $H^{2}(\Omega)$ с данньми Коши

$$
u_{0}^{+}(x)=0, \quad \partial_{\nu} u_{0}^{+}(x)=g(x) .
$$

Положим $v=u-u_{0}$. Тогда $v-$ решение задачи

$$
-\Delta v+V v=\lambda v+h \quad \text { в } \Omega, \quad \partial_{\nu} v^{+}=\mu v^{+},
$$

где $h=\Delta u_{0}-V u_{0}+\lambda u_{0} \in L_{2}(\Omega)$. Функция $v$ принадлежит $H^{2}(\Omega, B)$, и ее ряд Фурье по собственньм функщиям $u_{l}$ сходится к ней в $H^{2}(\Omega)$. Этот ряд имеет вид

$$
v(x)=\sum_{1}^{\infty}\left[\frac{\left(g, u_{l}^{+}\right)_{\Gamma}}{\lambda_{l}-\lambda}-\left(u_{0}, u_{l}\right)_{\Omega}\right] u_{l}(x)
$$

(см. (8)). Переходя на границу (с учетом того, что $\left.u_{0}^{+}=0\right)$, получаем

$$
u^{+}(x)=\sum_{1}^{\infty}\left[\frac{\left(g, u_{l}^{+}\right)_{\Gamma}}{\lambda_{l}-\lambda}-\left(u_{0}, u_{l}\right)_{\Omega}\right] u_{l}^{+}(x) .
$$


В отличие от (8) ряд (1.26) сходится в $H^{3 / 2}(\Gamma)$. В сушности мы улучшили сходимость ряда (8) вычитанием из него ряда $\sum\left(u_{0}, u_{l}\right)_{\Omega} u_{j}^{+}(x)\left(\right.$ сходящегося в $H^{t}(\Gamma)$ при $t<1$ ) с нулевой суммой.

Аналогичную регуляризацию допускает ряд вида (8) в случае общей задачи для системы $L u=0$ с граничным условием (1.4).

1.5. Ослабление предположений о гладкости. Пусть $k$ - целое неотрицательное число и $0<\gamma \leqslant 1$. Функция $a(x)$, определенная в некоторой области, по определению, принадлежит классу $C^{k, \gamma}$, если она непрерьвна и ограничена в замыкании этой области с производными до порядка $k-1$, а производные порядка $k$ сушествуют и удовлетворяют условию Гёльдера порядка $\gamma$ :

$$
\left|\partial^{\alpha} a(x)-\partial^{\alpha} a(y)\right| \leqslant C|x-y|^{\gamma}, \quad|\alpha|=k .
$$

В частности, класс $C^{0,1}$ состоит из функций, удовлетворяющих условию Липшица. Аналогично определяются классы функций $C^{k, \gamma}$ на $\Gamma$ (насколько это позволяет гладкость Г; при этом используются локальные координаты на Г). Граница Г принадлежит, по определению, $C^{k, 1}$, если локально она является графиком функции из $C^{k, 1}$.

Если граница области $\Omega$ принадлежит $C^{1,1}$, то функции из $\mathrm{H}^{2}(\Omega)$ имеют следы в $H^{3 / 2}(\Gamma)$, а их нормальные или конормальные производные - в $H^{1 / 2}(\Gamma)$. При этом оператор перехода к паре этих следов ограничен и имеет правый обратньй. См. [44; п. 1.5].

Мы сформулируем теорему об однозначной разрешимости наших задач в областях класса $C^{1,1}$ без предположения о самосопряженности. Сохранять предположения о бесконечной гладкости коэффициентов уже нет смысла, и мы предположсим, что все коэффициенты $a_{j k}(x)$ и $b_{j}(x)$ оператора $L$ принадлежсат $C^{0,1}$ в $\Omega$, а матрича $c(x)$ ограничена и измерима: $c(x) \in L_{\infty}(\Omega)$. Матричу $b(x)$ в граничном условии предположим принадлежащей $C^{0, \theta}(\Gamma)$ с $\theta>1 / 2$.

При этих предположениях оператор $(L, B)$ действует непрерьвно из $H^{2}(\Omega)$ в прямое произведение пространств $H^{0}(\Omega)$ и $H^{1 / 2}(\Omega)$. Коэрцитивность формы $\Phi$ на $H^{1}(\Omega)$ сохраняется; используя, если надо, сдвиг спектрального параметра, мы предположим, что $\lambda=0$, но

$$
\Phi(u, u) \geqslant C\|u\|_{1, \Omega}^{2}
$$

Оказывается, что тогда $(L, B)$ определяет изоморфизм, как при предположениях о бесконечной гладкости. Поясним, как получается этот результат.

Задача (1.1), (1.4) с $\lambda=0$ допускает следуюшую обобщенную, или слабую, постановку:

$$
\Phi(u, v)=(f, v)_{\Omega}+\left(g, v^{+}\right)_{\Gamma}, \quad v \in H^{1}(\Omega) .
$$

Пока мы предполагаем, что $f \in H^{0}(\Omega)$ и $g \in H^{-1 / 2}(\Gamma)$. (Более полным образом уравнение (1.29) будет обсуждаться в п. 2.2.) Следующее короткое рассуждение стандартно. Неравенство (1.28) позволяет принять форму $\Phi(u, v)$ за новое скалярное произведение в $H^{1}(\Omega)$, соответствуюшая норма эквивалентна обычной норме. Правая часть в (1.29) определяет непрерьвньй полулинейньй (или сопряженно-линейньй) функционал на $H^{1}(\Omega)$, и $u \in H^{1}(\Omega)$ однозначно определяется как функция, реализующая этот функционал в виде $\Phi(u, v)$. 
Далее доказывается гладкость этого решения: оно на самом деле принадлежит $H^{2}(\Omega)$. Это делается при помощи метода “тангенциальных разностных отношений" Ниренберга после локального вьпрямления границы при помоши преобразования координат класса $C^{1,1}$. См. [83; т. 4.16], где сильно эллиптические системы рассмотрены без предположения (1.3), а также [44; п. 2.2.2], где рассмотрено скалярное эллиптическое уравнение с вещественньми коэффициентами.

Итак, на области класса $C^{1,1}$ распространяются первые два утверждения теоремы 1.5. При усилении предположений о гладкости границы и коэффициентов утверждение о повышении гладкости решений усиливается, см., например, [83].

Переходя к оператору $\mathscr{L}$ при условиях формальной самосопряженности, мы видим, что он изоморфно отображает пространство $H^{2}(\Omega, B)$ на $H^{0}(\Omega)$. Поэтому мы так же, как в п. 1.3, получаем следующую теорему.

ТЕОРема 1.9. При сделанных в настоящем пункте предположениях оператор $\mathscr{L}$ имеет дискретный положительный спектр и ортонормированную в $H^{0}(\Omega)$ систему собственных функиий, которые принадлежсат $H^{2}(\Omega, B)$. Эта система остается безусловным базисом в $H^{t}(\Omega)$ при $0<t<3 / 2$ и в $H^{t}(\Omega, B)$ при $3 / 2<t \leqslant 2$.

Отсюда вытекают такие же следствия в отношении $R$-матрицы, как в предыдушем пункте.

Теперь кратко остановимся на асимптотике собственных значений. Результатов, относяшихся специально к областям класса $C^{1,1}$, автор не знает. Но для таких областей справедливы результаты, установленные для липшищевых областей. Забегая вперед (липшицевы области будут рассматриваться в следующем параграфе), отметим, что в липшицевых областях заведомо справедлива асимптотика в форме

$$
\lambda_{l}=C^{(n)} l^{2 / n}+o\left(l^{2 / n}\right)
$$

(см. [19]). Но в литературе имеются также результаты по уточнению оценки остатка. Некоторые подробности мы приведем в п. 2.8 .

ЗАмЕчАниЕ 1.10. Даже из асимптотики вида (1.30) вытекают сушественные следствия.

Согласно известной теореме Меньшова-Радемахера (см., например, [129; гл. XIII, т. 10.21]), если $c_{l}-$ коэффициенты Фурье функции $u(x)$ по ортонормированному базису $\left\{u_{l}(x)\right\}$, то условие

$$
\sum\left|c_{l}\right|^{2} \ln ^{2} l<\infty
$$

достаточно для сходимости ее ряда Фурье

$$
\sum c_{l} u_{l}(x)
$$

к $u(x)$ почти всюду. Поэтому из (1.30), используя малые степени оператора $\mathscr{L}$, получаем, что если функиия $u(x)$ принадлежит $H^{\varepsilon}(\Omega)$ со сколь угодно мальм положительным $\varepsilon$, то ряд (1.32) сходится $\kappa$ и $(x)$ почти всюду. Это известньй факт, и он справедлив даже если известна только односторонняя оценка $\lambda_{l} \geqslant C l^{2 / n}$. Из нее 
также получается оценка скорости сходимости в $H_{0}(\Omega)$ ряда Фурье (1.32) функции $u(x)$ из $H^{\varepsilon}(\Omega)$ :

$$
\left\|\sum_{p}^{q} c_{l} u_{l}(x)\right\|_{0, \Omega}^{2}=\sum_{p}^{q}\left|c_{l}\right|^{2} \leqslant C_{1} \lambda_{p}^{-2 \varepsilon}\|u\|_{\varepsilon, \Omega}^{2} \leqslant C_{2} p^{-4 \varepsilon / n}\|u\|_{\varepsilon, \Omega}^{2}
$$

(ср. [4; п. 6.1]). Здесь $0<\varepsilon<3 / 2$.

Аналогичные замечания об использовании асимптотики или оценок собственных чисел относятся и к дальнейшим параграфам.

Заканчивая этот пункт, отметим, что некоторое дальнейшее ослабление предположений о гладкости гранищы остается возможным: см. работу [63] и главу 7 в книге [80], а также указанные там ссылки.

1.6. Системы с кулоновскими особенностями. Следуя [96], мы можем использовать известное неравенство Харди

$$
\int|u(x)|^{2}|x|^{-2} d x \leqslant \widehat{C}_{n} \int|\nabla u(x)|^{2} d x \quad\left(u \in C_{0}^{\infty}\left(\mathbb{R}^{n}\right)\right)
$$

Оно доказано, например, в [30; гл. VI, $\S 5]$ для $n=3$, но доказательство с несложными поправками проходит и для бо́льших $n$. При $n=2$ неравенство (1.34) неверно (если не предполагать, что $u(0)=0)$, так как интеграл слева, вообще говоря, расходится. Поэтому ниже, говоря о кулоновских особенностях, мы всюду предполагаем, что $n \geqslant 3$.

Можно предположить, что оператор продолжения $E$ (см. чуть ниже п. 2.1) переводит функции, определенные в $\Omega$, в финитные функции. Поэтому из (1.34) следует, что оператор умножения на $1 /|x|$ действует ограниченным образом из $H^{1}(\Omega)$ в $H^{0}(\Omega)$, т.е. является мультипликатором из $H^{1}(\Omega)$ в $H^{0}(\Omega)$. Для операторов умножения на $1 /\left|x^{\alpha}-x^{\beta}\right|$ (см. п. 2 введения) получается то же самое, нужно только выбрать подходящий порядок интегрирования по разньм направлениям (см. [96]). Поэтому мы сделаем теперь такое предположение:

УСловиЕ IV. $c(x)=c_{1}(x)+c_{2}(x)$, әде $c_{1}(x)$ удовлетворяет прежсним предположениям относительно $c(x)$, а $\mathrm{c}_{2}(x)$ - мультипликатор из $H^{1}(\Omega)$ в $H^{0}(\Omega)$.

С этим предположением снова можно считать, что $n \geqslant 2$.

Посмотрим теперь, что сохраняется и что меняется в утверждениях предыдущих пунктов. Будет удобно обозначать невозмущенньй оператор (без слагаемого $c_{2} \cdot$ ) через $\mathscr{L}^{0}$ и возмушенньй оператор $\mathscr{L}^{0}+c_{2}$. через $\mathscr{L}$. Пусть для простоты матрица $c_{2}(x)$ эрмитова.

Оператор $\mathscr{L}$ изменился на слагаемое, действующее компактньм образом из $H^{2}(\Omega)$ в $\mathrm{H}^{0}(\Omega)$. Отсюда прежде всего следует, что он остался фредгольмовым оператором из $H^{2}(\Omega, B)$ в $H^{0}(\Omega)$. Далее, он остается самосопряженным полуограниченным снизу оператором в $H^{0}(\Omega)$ с областью определения $H^{2}(\Omega, B)$. Его спектр остается дискретным, и даже сохраняется асимптотика собственных значений с оценкой остатка, указанной в (1.25). Последнее утверждение будет объяснено в п. 2.8, см. там предложение 2.17 . 
В отношении гладкости собственных функций мы можем утверждать, что они принадлежат, по крайней мере, $H^{2}(\Omega)$. Действительно, достаточно применить к уравнению $\mathscr{L} u_{l}=\lambda_{l} u_{l}$ оператор, обратньй к $\mathscr{L}$.

Но если $c_{2}(x)$ имеет только кулоновские особенности, то $u_{l} \in C^{\infty}$ вне этих особенностей в силу известной теоремы о локальной гладкости эллиптических задач.

Осталось понять, что сохраняются свойства базисности собственных функций. Оператор $\mathscr{L}$, как мы уже отметили, по-прежнему полуограничен снизу; предположив без ограничения обшности, что он имеет положительный спектр, можно воспользоваться его степенями. Поэтому базисность системы собственных функций в $H^{t}(\Omega)$, $0 \leqslant t<3 / 2$, получается так же, как в случае отсутствия слагаемого $c_{2} \cdot$. При $3 / 2<t \leqslant 2$ сохраняется базисность в $H^{t}(\Omega, B)$. Аналогично обстоит дело, если $\Omega-$ область с границей класса $C^{1,1}$ (см. п. 1.5).

\section{§2. Задачи в области с липшицевой границей}

2.1. Соболевские пространства в липшицевой области и на ее границе. Переходя к случаю липшищевой области $\Omega$, мы сначала сформулируем основные свойства пространств $H^{t}(\Omega)$ и $H^{t}(\Gamma)$ в этом случае. Это достаточно деликатная тема в некоторых ее пунктах. Для справок и дополнительной информации можно использовать книги [18], [44] и [83].

Общеизвестно определение пространства $H^{t}\left(\mathbb{R}^{n}\right)(t \in \mathbb{R})$ : это пополнение линеала $C_{0}^{\infty}\left(\mathbb{R}^{n}\right)$ по норме, определяемой равенством

$$
\|u\|_{t, \mathbb{R}^{n}}^{2}=\int\left(1+|\xi|^{2}\right)^{t}|F u(\xi)|^{2} d \xi
$$

где $F$ - преобразование Фурье. Эта норма сохраняет смысл для элементов из $H^{t}\left(\mathbb{R}^{n}\right)$, если понимать преобразование Фурье в смысле обобщенных функций.

Пусть теперь $\Omega$ - липшицева область, для простоты со связной компактной границей. Пространство $H^{t}(\Omega)$ состоит из обобщенных функций $u$ в $\Omega$ с конечной нормой, определяемой равенством

$$
\|u\|_{k, \Omega}^{2}=\int_{\Omega} \sum_{|\alpha| \leqslant k}\left|\partial^{\alpha} u(x)\right|^{2} d x
$$

при целом $t=k \geqslant 0$ и равенством

$$
\|u\|_{t, \Omega}^{2}=\|u\|_{k, \Omega}^{2}+\int_{\Omega} \int_{\Omega} \sum_{|\alpha|=k} \frac{\left|\partial^{\alpha} u(x)-\partial^{\alpha} u(y)\right|^{2}}{|x-y|^{n+2 \theta}} d x d y
$$

при $t=k+\theta, 0<\theta<1$. Если $\Omega=\mathbb{R}^{n}$, то эти нормы эквивалентны норме, указанной в (2.1).

При любом $t>0$ существует линейньй оператор $E$ продолжения функций из $H^{t}(\Omega)$, $0 \leqslant t<t_{0}$, до функций из $H^{t}\left(\mathbb{R}^{n}\right)$, действуюший ограниченным образом из первого из этих пространств во второе и не зависящий от $t$ (см. [118; гл. VI, §3]). При $t_{0}=1 / 2$ можно просто положить $E u(x)=0$ вне $\Omega$. 
При всех $t>0$ пространство $\left[H^{t}(\Omega)\right]^{*}$, сопряженное к $H^{1}(\Omega)$, можно отождествить с пространством $H_{\bar{\Omega}}^{-t}\left(\mathbb{R}^{n}\right)$ (см., например, [83; т. 3.29 и т. 3.30]). Последнее является подпространством в $H^{-t}\left(\mathbb{R}^{n}\right)$ и состоит из тех входяших в него обобщенных функций в $\mathbb{R}^{n}$, носители которых содержатся в $\bar{\Omega}$. При этом двойственность определяется следуюшим образом. Если $v \in H^{t}(\Omega)$ и $f \in H_{\bar{\Omega}}^{-t}\left(\mathbb{R}^{n}\right)$, то, по определению,

$$
(f, v)_{\Omega}=(f, E v)_{0, \mathbb{R}^{n}}
$$

Это выражение не зависит от выбора оператора $E$ и является продолжением скалярного произведения в $H^{0}(\Omega)$ на прямое произведение пространств $H_{\bar{\Omega}}^{-t}\left(\mathbb{R}^{n}\right)$ и $H^{t}(\Omega)$. Для простоты вместо $H_{\bar{\Omega}}^{-t}\left(\mathbb{R}^{n}\right)$ мы дальше будем писать $H_{0}^{-t}(\Omega)$.

Пространства $H^{t}(\Omega)(t \geqslant 0)$ расширяются с уменьшением $t$, и соответствуюшие вложения компактны. Поэтому пространства $\left[H^{t}(\Omega)\right]^{*}=H_{0}^{-t}(\Omega)$ расширяются с pостом $t$, и соответствующие вложения тоже компактны. В обоих случаях более узкое пространство плотно в более широком. При $t=0$ шкалы этих пространств смыкаются на пространстве $L_{2}(\Omega)$. Если $t^{\prime}>0$ и $t^{\prime \prime}>0$, то вложение пространства $H^{t^{\prime}}(\Omega)$ в пространство $H_{0}^{-t^{\prime \prime}}(\Omega)$ осушествляется следуюшим образом: функция $u(x)$ из $H^{t^{\prime}}(\Omega)$ продолжается нулем вне $\Omega$.

Обе шкалы являются интерполящионными (как в смысле комплексной, так и в смысле вещественной интерполяции); например,

$$
\left[H^{t_{0}}(\Omega), H^{t_{1}}(\Omega)\right]_{\theta}=\left[H^{t_{0}}(\Omega), H^{t_{1}}(\Omega)\right]_{\theta, 2}=H^{t_{2}}(\Omega)
$$

при $0<\theta<1$, где $t_{2}=(1-\theta) t_{0}+\theta t_{1}$. Здесь $[\cdot, \cdot]_{\theta}$ и $[\cdot, \cdot]_{\theta, 2}-$ соответственно комплексная и вешественная интерполяции; см. определения, например, в [17].

При $t>0$ пространство $H_{0}^{t}(\Omega)=H_{\bar{\Omega}}\left(\mathbb{R}^{n}\right)$ можно определить так же, как вьше при $t<0$. Его можно отождествить с пространством функций $u$ в $\Omega$, продолжения которых нулем вне $\Omega$ принадлежат $H^{t}\left(\mathbb{R}^{n}\right)$.

Пространство $H^{t, 0}(\Omega)$ определим для $t>0$ как пополнение линеала $C_{0}^{\infty}(\Omega)$ в $H^{t}(\Omega)$. Это пространство можно отождествить с $H_{0}^{t}(\Omega)$ (и соответствующие нормы эквивалентны) при всех $t$, кроме полуцелых значений $1 / 2,3 / 2, \ldots$, а при этих полуцелых $t$ имеет место включение $H_{0}^{t}(\Omega) \subset H^{t, 0}(\Omega)$ (см., например, [83; т. 3.33]).

Пространства $H^{-t}(\Omega)$ с $t \geqslant 0$ можно сразу определить как сопряженные к $H_{0}^{t}(\Omega)$ относительно продолжения скалярного произведения в $H^{0}(\Omega)$ на прямое произведение этих пространств. Это тоже интерполящионные шкалы с компактньми вложениями, и более узкое пространство плотно в более широком. При $0 \leqslant t<1 / 2$ имеет место совпадение

$$
H^{ \pm t}(\Omega)=H_{0}^{ \pm t}(\Omega)
$$

(cp. [44; сл. 1.4.4.5]). При $t=0$ эти шкалы тоже смыкаются на пространстве $L_{2}(\Omega)$. Линеал $C_{0}^{\infty}(\Omega)$ всюду плотен в $H_{0}^{t}(\Omega)$ при любом $t \in \mathbb{R}^{n}$ (см. [83; т. 3.29]).

Оператор умножения на функцию из $C^{k-1,1}(\bar{\Omega})$ действует ограниченным образом в $H^{t}(\Omega)$ и $H_{0}^{t}(\Omega)$ при $|t| \leqslant k$.

Оператор дифференцирования $\partial_{j}$ в смысле обобщенных функций в $\Omega$ действует ограниченным образом из $H^{t}(\Omega)$ в $H^{t-1}(\Omega)$ при $t \neq 1 / 2$ (см. [44; сл. 1.4.4.6]). 
Пространства $H^{t}(\Gamma)$ инвариантно определены только при $|t| \leqslant 1$. Определение обычным образом использует разбиение единищы и локальные координатына Г (с липшицевыми преобразованиями координат).

Оператор умножения на функцию из $C^{0, \theta}(\Gamma), \theta>1 / 2$, действует ограниченньм образом в $H^{t}(\Gamma)$ при $|t| \leqslant 1 / 2$.

Оператор $u \mapsto u^{+}$перехода к следу (граничному значению) на Г действует ограниченным образом из $H^{t}(\Omega)$ в $H^{t-1 / 2}(\Gamma)$ при $1 / 2<t<3 / 2$ (см. [28], [35], [83]). При $t=3 / 2$, в отличие от случая гладкой границы, это неверно, пространство следов шиpe, чем $H^{1}(\Gamma)$ (см. [53] и указанные там ссылки). Но функции из $H^{t}(\Omega)$ с $t>3 / 2$ имеют следы в $H^{1}(\Gamma)$ (см. [35]). Кроме того, дополнительными свойствами обладают следы поверхностных потенциалов, о чем мы будем говорить ниже в $\S 5$. Отметим также, что указанный в первой фразе этого абзаца оператор имеет правый обратный.

При $t>n / 2$ пространство $H^{t}(\Omega)$ непрерывно вложено в пространство $C(\bar{\Omega})$ функций, непрерывных в $\bar{\Omega}$, и при $n=2,1 / 2<t \leqslant 1$ пространство $H^{t}(\Gamma)$ непрерьвно вложено в пространство $C(\Gamma)$ функщий, непрерьвных на $\Gamma$.

Пространство $H_{\mathrm{loc}}^{t}\left(\Omega^{-}\right)$состоит из функций, сужения которых на $\Omega_{R}^{-}$принадлежат $H^{t}\left(\Omega_{R}^{-}\right)$при сколь угодно большом $R$. Множество в $H_{\text {loc }}^{t}\left(\Omega^{-}\right)$назьвается ограниченным, если сужения функций из него на $\Omega_{R}^{-}$образуют ограниченное множество в $H^{t}\left(\Omega_{R}\right)$ при сколь угодно большом $R$.

2.2. Существование и гладкость обобщенных решений. Липшицева функция почти всюду дифференцируема (теорема Радемахера, см. [127; гл. Х, п. 11] или [118; гл. VIII]), поэтому касательная плоскость и нормаль существуют на липшицевой поверхности почти всюду. Значит, граничное условие $B u=g$ формально сохраняет смысл. Но непосредственно придать ему неформальный смысл непросто, поэтому обычно рассматривают обобшенную постановку (1.29). В частности, эта точка зрения принята в работе [28]. Другой подход, с поверхностными потенциалами, мы откладьваем до главы II. Коэффициенты $a_{j k}, b_{j}, c, b$ подчиним таким же предположениям, как в п. 1.5. Сдвигая, если потребуется, спектральньй параметр, предположим, что выполнено неравенство (1.28). Пока мы не предполагаем систему формально самосопряженной.

Естественные предположения о пространствах, к которым принадлежат функции $u, f$ и $g$, состоят в следуюшем: $u \in H^{1}(\Omega), f \in H_{0}^{-1}(\Omega), g \in H^{-1 / 2}(\Gamma)$. Более точно, дело обстоит следующим образом (ср. [83]).

1. При заданных $f$ и $g$ (в указанных пространствах) однозначно определяется $и$. Действительно, как в п. 1.4, правая часть в (1.29) - непрерьвньй полулинейньй функционал над $H^{1}(\Omega)$, и в силу $(1.28)$ он реализуется в виде $\Phi(u, v)$ с однозначно определенным $u \in H^{1}(\Omega)$. Более того, оператор $(f, g) \mapsto u$ ограничен. Это исходная (и по сушеству классическая) теорема о существовании и единственности обобщенного решения.

2. При заданньх и и о однозначно определяется $f$. Действительно, $(f, v)_{\Omega}$ выражается через остальные члены формулы (1.29) и $f$ однозначно определяется как соответствуюший элемент пространства, сопряженного к $H^{1}(\Omega)$. Более того, оператор $(u, g) \mapsto f$ ограничен.

3. Соотношение $(1.29)$ с $v \in H_{0}^{1}(\Omega)$ равносильно тому, что $L u=f$ внутри $\Omega$ в смысле обобщенных функций: это следует из того, что $C_{0}^{\infty}(\Omega)$ плотно в $H_{0}^{1}(\Omega)$. Пред- 
положим, что это условие выполнено. Тогда полное соотношение (1.29) однозначно определяет $g$ по $u$ и $f$. Действительно, пусть $w \in H^{1 / 2}(\Gamma)$. Определив $v \in H^{1}(\Omega)$ так, что $v^{+}=w$, мы видим, что $(g, w)_{\Gamma}$ определяется формулой $(1.29)$ как непрерьвный линейный функционал над $H^{1 / 2}(\Gamma)$, так что $g$ определяется, и причем однозначно, как соответствующий элемент из $H^{-1 / 2}(\Gamma)$. (В частности, сказанное означает, что решение уравнения $L u=f$ из $H^{1}(\Omega)$ имеет однозначно определенное значение конормальной производной $\partial_{\nu_{a}} u^{+}{ }_{\text {в }} H^{-1 / 2}(\Gamma)$.) Более того, видно, что оператор $(u, f) \mapsto g$ ограничен.

4. Однако если мы задали только $u$, то $f$ однозначно определяется только как обобщенная функция $L u$ внутри $\Omega$. Это непрерьвньй функционал над $H_{0}^{1}(\Omega)$. Остается произвол в выборе его продолжения на $H^{1}(\Omega)$. Оно полностью определяется, если задать $g \in H^{-1 / 2}(\Gamma)$ (см. п. 2). Таким образом, отображение $(f, g) \mapsto u$ не имеет однозначного обратного. Это, так сказать, наша "плата" за обобщенную постановку задачи. Но если $g=0$, то $f$ определяется по и однозначно. Более того, можно предположить, что $g=0$, не ограничивая обшности, так как $\left(g, v^{+}\right)_{\Gamma}-$ полулинейнњй функционал над $H^{1}(\Omega)$, можно реализовать его в виде $\left(f_{1}, v\right)_{\Omega}$ с $f_{1} \in H_{0}^{-1}(\Omega)$ и прибавить $f_{1} \mathrm{k} f$, не меняя правой части в (1.29). Кроме того, если $f=0$ (в частности, $L u=0$ в $\Omega)$, то однозначно определяется $g$.

Теперь сформулируем теорему о гладкости решений.

Теорема 2.1. Пусть и новке (1.29) из $\mathrm{H}^{1}(\Omega)$, но $f \in H_{0}^{t-1}(\Omega)$ и $g \in H^{t-1 / 2}(\Gamma)$, где $0<t<1 / 2$. Тогда $u \in H^{t+1}(\Omega)$.

Это аналог результата Саваре в [111] для скалярного уравнения

$$
\operatorname{div} A(x) \operatorname{grad} u(x)=f(x)
$$

с вещественной симметричной матрицей $A(x)$ при условии Неймана. Но в формулировке Саваре нет точного указания пространства, которому принадлежит $f$. Кроме того, в отличие от [111] мы рассматриваем систему с комплексными коэффициентами (и комплексные решения).

Метод доказательства - тот же, что и в [111]. В [111] отмечено, что это по существу развитие метода тангенциальных разностных отношений Ниренберга; но оно далеко не тривиально. Ввиду важности результата мы проведем полное доказательство (в п. 2.4 после необходимой для этого подготовки в п. 2.3). В [111] рассмотрены также нелинейные уравнения, чего мы делать не будем; это позволит несколько облегчить изложение.

2.3. Некоторые пространства Бесова и формальные правила их интерполяции. Пространства Бесова $B_{2, q}^{s}(\Omega), 1 \leqslant q \leqslant \infty, s \geqslant 0$, образуют некоторое расширение класса соболевских пространств $H^{s}(\Omega)$, так как $B_{2,2}^{s}(\Omega)=H^{s}(\Omega)$. Ниже по существу нужны только пространства $B_{2, \infty}^{s}(\Omega)$ при $s=1 / 2$ и $3 / 2$. Возможны разные эквивалентные определения этих пространств, в частности, следующие.

Пусть $\Omega_{d}, d>0,-$ подмножество точек в $\Omega$, отстояших от $Г$ больше чем на $d$. Пусть $D$ - множество, порождающее $\mathbb{R}^{n}$ и звездное относительно начала координат 0 . Для 
$s \in(0,1)$ определим полунорму $[u]_{s, \Omega}$ равенством

$$
[u]_{s, \Omega}^{2}=\sup _{h \in D \backslash 0}|h|^{-2 s} \int_{\Omega_{|h|}}|u(x+h)-u(x)|^{2} d x .
$$

Пространство $B_{2, \infty}^{s}(\Omega)$ состоит из принадлежаших $H^{0}(\Omega)$ функций с конечной нормой $\|u\|_{0, \Omega}+[u]_{s, \Omega}$. Пространство $B_{2, \infty}^{1+s}(\Omega)$ состоит из функций из $H^{1}(\Omega)$ с конечной нормой $\|u\|_{1, \Omega}+[\operatorname{grad} u]_{s, \Omega}$.

Отметим, что при $0<s<1$ справедливы следуюшие интерполяционные формулы (cp. [111]):

$$
\begin{gathered}
{\left[H^{1}(\Omega), L_{2}(\Omega)\right]_{s, \infty}=B_{2, \infty}^{1-s}(\Omega),} \\
{\left[H^{1}(\Omega), B_{2, \infty}^{3 / 2}(\Omega)\right]_{s, 2}=H^{1+s / 2}(\Omega) .}
\end{gathered}
$$

Свойства упомянутых пространств $B_{2, \infty}^{s}(\Omega)$ во многом похожи на свойства пространств $H^{s}(\Omega)$. В частности, если $\sum \varphi_{j}(x)$ - конечное разбиение единицы в $\bar{\Omega}$, состоящее из бесконечно гладких функций, то $u$ принадлежит пространству $B_{2, \infty}^{s}(\Omega)$ тогда и только тогда, когда ему принадлежат все $\varphi_{j} u$, при этом сумма норм этих функций эквивалентна норме функции $u$. Но пространства $B_{2, \infty}^{s}(\Omega)$ не сепарабельны (см. [18]). В отношении определений более общих пространств Бесова и их свойств отсылаем читателя к книгам [18], [17] и статьям [53], [39], [92], [95]; см. также приведенные там ссылки.

Отметим еще три свойства вещественной интерполяции. Первое из них - это предложение 4.2 из [111] (см. также ссылку в [111]). Второе - нужньй случай теоремы реитерации, полную формулировку см. в [17; п. 3.5]. Третье - утверждение о двойственности, полную формулировку см. там же в п. 3.7 .

1. Пусть $X, Y$ и $Z$ - три банаховых пространства, $X$ непрерьвно вложено в $Y$ и $T$ - линейньй ограниченньй оператор из $X$ в $Z$. Предположим, что при некоторых постоянных $C_{1}>0$ и $\sigma \in(0,1)$ справедливо неравенство

$$
\|T u\|_{Z} \leqslant C_{1}\|u\|_{X}^{1-\sigma}\|u\|_{Y}^{\sigma} \quad(u \in X) .
$$

Тогда $T$ непрерывно продолжается до ограниченного оператора из $[X, Y]_{\sigma, 1}$ в $Z$.

2. Пусть $X_{0}$ и $X_{1}$ - два банаховых пространства и для простоты одно из них непрерьвно вложено в другое. Пусть $0 \leqslant \theta_{j} \leqslant 1, \theta_{0} \neq \theta_{1}, 1 \leqslant q_{j} \leqslant \infty, 0<\eta<1$, $1 \leqslant q \leqslant \infty$. Тогда

$$
\left[\left[X_{0}, X_{1}\right]_{\theta_{0}, q_{0}},\left[X_{0}, X_{1}\right]_{\theta_{1}, q_{1}}\right]_{\eta, q}=\left[X_{0}, X_{1}\right]_{\theta, q},
$$

где $\theta=(1-\eta) \theta_{0}+\eta \theta_{1}$. Здесь $\left[X_{0}, X_{1}\right]_{\theta_{j}, q_{j}}$ заменяется на $X_{0}$ при $\theta_{j}=0$ и на $X_{1}$ при $\theta_{j}=1$.

3. Пусть одно из пространств $X, Y$ непрерывно вложено в другое и плотно в нем. Тогда при $0<\theta<1,1 \leqslant q<\infty, q^{-1}+\left(q^{\prime}\right)^{-1}=1$ справедливо соотношение

$$
\left[X^{*}, Y^{*}\right]_{\theta, q^{\prime}}=\left\{[X, Y]_{\theta, q}\right\}^{*} .
$$


2.4. Доказательство теоремы 2.1. Прежде всего мы должны напомнить свойство конуса липшицевой поверхности. Известно, что замкнутая поверхность липшицева тогда и только тогда, когда она удовлетворяет равномерному условию конуса (см. [44; т. 1.2.2.2]), т.е. каждая точка $x$ на Г является вершиной двух замкнутых конусов $C^{ \pm}(x)$ с общей осью, конгруэнтных фиксированному конусу и таких, что все их точки, кроме $x$, лежат соответственно в $\Omega^{ \pm}$. Эти конусы можно подчинить некоторому условию регулярности; его полная формулировка довольно громоздка (см., например, [123]), но сейчас для нас важно лишь, что направление оси этих конусов можно считать непрерьвно зависяшим от $x$. В дальнейшем такое семейство предполагается фиксированным. Здесь нам понадобятся конусы $C^{+}(x)$.

ДоКАЗАТЕЛЬСТВо ТЕОРЕМЫ 2.1. Итак, рассмотрим задачу $(1.1),(1.4)$ в липшицевой области $\Omega$. Младшие члены в уравнении, если они есть, можно перенести к правой части; так как мы предполагаем, что рассматриваемое решение $u(x)$ принадлежит $H^{1}(\Omega)$, то к правой части добавится функция из $H^{0}(\Omega)$. Это позволяет предположить, не ограничивая обшности, что младшие члены состоят из единственного слагаемого $\alpha u$ с настолько большим $\alpha>0$, что

$$
\Phi_{0}(v, v)+\alpha(v, v)_{\Omega} \geqslant C_{2}\|v\|_{1, \Omega}^{2} \quad\left(v \in H^{1}(\Omega)\right) .
$$

По аналогичной причине можно предположить, что в граничном условии $b(x)=0$. Поэтому будем рассматривать задачу Неймана. Старшие коэффициенты $a_{j k}^{r s}(x)$ в уравнении удобно предположить продолженньми во внешнюю граничную полоску с сохранением их свойств: условия симметрии (1.3), условия II и равномерного условия Липшица. Пусть $\varepsilon$ - такое положительное число, что $\overline{O_{\varepsilon}\left(x_{0}\right)}$ содержится в объединении $\bar{\Omega}$ с этой граничной полоской для любой точки $x_{0} \in \Gamma$.

Теперь, как в [111], будем доказывать следующее ключевое предложение.

ПРЕДЛОЖЕНИЕ 2.2. Пусть $и(x)$ - обобщенное решение задачи

$$
L_{0} u+\alpha u=f \quad \text { в } \quad \Omega, \quad \partial_{a} u^{+}=0 \quad \text { на } \quad \Gamma,
$$

принадлежсащее $H^{1}(\Omega)$, и пусть $f \in H^{0}(\Gamma)$. Тогда и $(x)$ принадлежсит пространству Бесова $B_{2, \infty}^{3 / 2}(\Omega)$ и справедлива оценка

$$
\|u\|_{B_{2, \infty}^{3 / 2}(\Omega)}^{2} \leqslant C_{3}\|f\|_{0, \Omega}\|u\|_{1, \Omega}
$$

с не зависящей от и постоянной $C_{3}$.

ДокАЗАТЕЛЬСтво. 1. В силу (2.12) задача (2.13) имеет единственное решение в $H^{1}(\Omega)$. Введем формы

$$
\Phi_{\alpha}(u)=\Phi_{0}(u, u)+\alpha(u, u)_{\Omega}, \quad \Psi(u)=\Phi_{\alpha}(u)-2 \operatorname{Re}(f, u)_{\Omega} .
$$

Функция $u(x)$ комплекснозначная, но форма $\Phi_{0}(u, v)$ эрмитова, поэтому значения формы $\Psi(u)$ вещественны. На решении $u(x)$ она принимает свое наименьшее значение. Действительно, пусть $v$ тоже принадлежит $H^{1}(\Omega)$; тогда

$$
\Psi(u+v)-\Psi(u)=\Phi_{\alpha}(v)+2 \operatorname{Re}\left[\Phi_{0}(u, v)+\alpha(u, v)_{\Omega}-(f, v)_{\Omega}\right]
$$


и здесь выражение в квадратных скобках равно нулю. В силу (2.12) имеет место неравенство

$$
C_{2}\|v\|_{1, \Omega}^{2} \leqslant \Psi(u+v)-\Psi(u) .
$$

Дальнейший план состоит, грубо говоря, в проверке того, что если взять $v(x)=$ $u(x+h)-u(x)$, то правую часть в $(2.16)$, деленную на $|h|$, удается оценить через правую часть в (2.14) с не зависящей от $h$ постоянной. На самом деле требуется более ювелирная работа с локализацией сдвига на $h$ и притом сдвига внутрь области $\Omega$. Мы далее проводим ее, следуя [111].

2. Введем ряд обозначений. Пусть $x_{0}-$ любая точка сначала на Г. Зафиксируем ее. Через $D=D\left(x_{0}\right)$ обозначим множество таких векторов $h$ в $\mathbb{R}^{n}$, выходяших из начала, что $x_{0}+h \in C^{+}\left(x_{0}\right)$ при $h \in D\left(x_{0}\right)$. Для $\rho>0$ обозначим через $\Omega_{\rho}=\Omega_{\rho}\left(x_{0}\right)$ пересечение $\Omega^{+} \cap O_{\rho}\left(x_{0}\right)$. Пусть $r_{0}$ - высота конусов $C^{+}\left(x_{0}\right)$. Можно предположить, что сушествует число $\rho=\rho\left(x_{0}\right) \in\left(0, r_{0}\right)$ со следующим свойством: из $x \in O_{2 \rho}\left(x_{0}\right)$, $h \in D\left(x_{0}\right),|h| \leqslant \rho$ следует, что $x+h \in \Omega$. Зафиксируем такое $\rho$. (Его можно сразу предположить не зависящим от $x_{0} \in \Gamma$, но это несущественно.) Добавочно будем считать, что $\rho<\varepsilon / 3$.

Для функции $v(x)$, определенной в $\Omega$, через $v_{0}(x)$ обозначим ее продолжение на $\mathbb{R}^{n}$ для определенности нулем вне $\Omega^{+}$и положим $v_{h}(x)=v_{0}(x+h)$. Зафиксируем вещественную скалярную функцию $\varphi(x)=\varphi_{x_{0}}(x)$ из $C_{0}^{\infty}\left(\mathbb{R}^{n}\right)$ со следуюшими свойствами: $0 \leqslant \varphi(x) \leqslant 1, \varphi(x)=1$ при $\left|x-x_{0}\right| \leqslant \rho$ и $\varphi(x)=0$ при $\left|x-x_{0}\right| \geqslant 2 \rho$. (Эти функции можно предположить сдвигами $\psi\left(x-x_{0}\right)$ фиксированной функции $\psi(x) \in C_{0}^{\infty}\left(\mathbb{R}^{n}\right)$.) Положим, далее,

$$
\mathscr{T}_{h} v(x)=\varphi(x) v_{h}(x)+[1-\varphi(x)] v(x) .
$$

Через $\zeta(x)$ обозначим матричную функцию с компонентами $\zeta_{j}^{r}(x)(1 \leqslant j \leqslant n$, $1 \leqslant r \leqslant m)$. В частности, если $\zeta_{j}^{r}(x)=\partial_{j} v_{r}(x)$, то будем писать $\zeta(x)=\nabla v(x)$. Аналогично (2.17) определим

$$
\mathscr{T}_{h} \zeta(x)=\varphi(x) \zeta_{h}(x)+[1-\varphi(x)] \zeta(x)
$$

$\mathrm{B}(2.17)$ и $(2.18) h \in D\left(x_{0}\right),|h| \leqslant \rho$. Наконец, положим $F(x, \zeta)=\sum a_{j k}^{r s}(x) \zeta_{k}^{s} \overline{\zeta_{j}^{r}}$. Заметим, что это квадратичная неотрицательная форма от $(\xi, \eta)$, где $\zeta=\xi+i \eta$, поэтому она вьпукла по $(\xi, \eta)$ (см., например, $[108 ; \S 4])$.

Из (2.16) следует, что для решения $u$ рассматриваемой задачи

$$
C_{2}\left\|\mathscr{T}_{h} u-u\right\|_{1, \Omega}^{2} \leqslant \Psi\left(\mathscr{T}_{h} u\right)-\Psi(u)
$$

где постоянная $C_{2}$ не зависит от $h . \mathrm{B}$ дальнейших неравенствах в этом доказательстве $C_{j}$ также не зависят от $h$ (и можно считать, что они не зависят от $x_{0}$ ). Мы теперь должны показать, что для всех $v \in H^{1}\left(\Omega^{+}\right)$

$$
\Psi\left(\mathscr{T}_{h} v\right)-\Psi(v) \leqslant C_{4}|h|\|v\|_{1, \Omega_{3 \rho}}\left(\|v\|_{1, \Omega_{3 \rho}}+\|f\|_{0, \Omega_{3 \rho}}\right) .
$$

Здесь и далее в доказательстве постоянные не зависят и от рассматриваемых функций. Сначала будем доказывать такое неравенство для $\Phi_{0}$ вместо $\Psi$. 
3. Заметим, что если $\operatorname{supp} \zeta(x) \subset \Omega_{2 \rho}$, то в силу неравенства Шварца

$$
\int_{\Omega}[F(x, \sigma(x)+\zeta(x))-F(x, \sigma(x))] d x \leqslant C_{5}\|\zeta\|_{0, \Omega_{2 \rho}}\left(\|\zeta\|_{0, \Omega_{2 \rho}}+\|\sigma\|_{0, \Omega_{2 \rho}}\right) .
$$

В силу выпуклости формы $F$

$$
\begin{aligned}
F\left(x, \mathscr{T}_{h} \zeta(x)\right)-F(x, \zeta(x)) & \leqslant \varphi(x) F\left(x, \zeta_{h}(x)\right)+[1-\varphi(x)] F(x, \zeta(x))-F(x, \zeta(x)) \\
& =\varphi(x)\left[F\left(x, \zeta_{h}(x)\right)-F(x, \zeta(x))\right]
\end{aligned}
$$

поэтому

$$
\begin{aligned}
\int_{\Omega^{+}} & {\left[F\left(x, \mathscr{T}_{h} \zeta(x)\right)-F(x, \zeta(x))\right] d x \leqslant \int_{\Omega_{2 \rho}} \varphi(x)\left[F\left(x, \zeta_{h}(x)\right)-F(x, \zeta(x))\right] d x } \\
= & \int_{\Omega_{2 \rho}+h} \varphi(x-h) F(x, \zeta(x)) d x-\int_{\Omega_{2 \rho}} \varphi(x) F(x, \zeta(x)) d x \\
= & \int_{\Omega_{2 \rho}+h}[\varphi(x-h)-\varphi(x)] F(x, \zeta(x)) d x \leqslant C_{6}|h|\|\zeta\|_{0, \Omega_{3 \rho}}^{2} .
\end{aligned}
$$

Так как $\nabla \mathscr{T}_{h} v=\mathscr{T}_{h} \nabla v+\left(v_{h}-v\right) \nabla \varphi$, то

$$
\begin{aligned}
\Phi_{0}\left(\mathscr{T}_{h} v\right)-\Phi_{0}(v)= & \int_{\Omega}\left[F\left(x, \mathscr{T}_{h} \nabla v+\left(v_{h}-v\right) \nabla \varphi\right)-F\left(x, \mathscr{T}_{h} \nabla v\right)\right] d x \\
& +\int_{\Omega}\left[F\left(x, \mathscr{T}_{h} \nabla v\right)-F(x, \nabla v)\right] d x
\end{aligned}
$$

Здесь второе слагаемое справа оценивается при помощи (2.23), а первое - при помощи (2.21) и неравенства

$$
\left\|v_{h}-v\right\|_{0, \Omega_{2 \rho}} \leqslant|h|\|\nabla v\|_{0, \Omega_{3 \rho}},
$$

которое получается из формулы

$$
v(x+h)-v(x)=\sum_{1}^{n} h_{j} \int_{0}^{1} \partial_{j} v(x+t h) d t
$$

при помоши неравенства Шварца и теоремы Фубини. В итоге получаем

$$
\Phi_{0}\left(\mathscr{T}_{h} v\right)-\Phi_{0}(v) \leqslant C_{7}|h|\|v\|_{1, \Omega_{3 \rho}}^{2}
$$

- неравенство вида $(2.20)$ для $\Phi_{0}(v)$.

Для функционала $(v, v)_{\Omega}$ с использованием его вьпуклости и неравенства $(2.25)$ тоже получается оценка вида (2.20):

$$
\begin{aligned}
\int_{\Omega^{+}} & \left(\mathscr{T}_{h} v \cdot \mathscr{T}_{h} \bar{v}-v \cdot \bar{v}\right) d x \leqslant \int_{\Omega} \varphi(x)\left(v_{h} \cdot \bar{v}_{h}-v \cdot \bar{v}\right) d x \\
\quad= & \int_{\Omega_{2 \rho}} \varphi(x)[v(x+h)-v(x)] \cdot \bar{v}(x+h)+v(x) \cdot[\bar{v}(x+h)-\bar{v}(x)] d x \\
\quad \leqslant & C_{8}|h|\|v\|_{1, \Omega_{3 \rho}}^{2} .
\end{aligned}
$$


Она получается также для функционала $(f, v)_{\Omega}$ :

$$
\int_{\Omega^{+}} f \cdot\left(\mathscr{T}_{h} \bar{v}-\bar{v}\right) d x \leqslant C_{9}|h|\|f\|_{0, \Omega_{2 \rho}}\|\nabla v\|_{0, \Omega_{3 \rho}} .
$$

Из (2.26)-(2.28) получаем (2.20).

4. Конструкция операторов $\mathscr{T}_{h}$ и результат (2.20) распространяются на внутренние точки $x_{0} \in \Omega^{+}$. Из покрытия замыкания области $\Omega$ открытыми шарами $O_{\rho}$ можно выбрать конечное. Для оставшихся шаров все постоянные можно считать обшими. Нормы по $\Omega_{3 \rho}$ не превосходят нормы по $\Omega$. С учетом того, что

$$
\|u\|_{1, \Omega} \leqslant C_{10}\|f\|_{H_{0}^{-1}(\Omega)} \leqslant C_{11}\|f\|_{0, \Omega}
$$

(см. п. 2.2), а также определений в п. 2.3 это приводит к следующему результату:

$$
\|\operatorname{grad} u\|_{B_{2, \infty}^{1 / 2}(\Omega)}^{2} \leqslant C_{12}\|f\|_{0, \Omega}\|u\|_{1, \Omega} .
$$

Этим предложение 2.2 доказано.

5. Из него снова с использованием (2.29) следует, что при $f \in H^{0}(\Omega)$

$$
\|u\|_{B_{2, \infty}^{3 / 2}(\Omega)}^{2} \leqslant C_{13}\|f\|_{0, \Omega}\|f\|_{H_{0}^{-1}(\Omega)} .
$$

Отсюда в силу утверждения 1 из п. 2.3 следует, что $\mathscr{L}^{-1}$ - ограниченньй оператор из пространства $\left[\left(H^{1}(\Omega)\right)^{*}, L_{2}(\Omega)\right]_{1 / 2,1}$ в пространство $B_{2, \infty}^{3 / 2}(\Omega)$. Кроме того, как мы знаем, это ограниченный оператор из $H_{0}^{-1}(\Omega)$ в $H^{1}(\Omega)$. Значит, это ограниченный оператор

$$
\left[H_{0}^{-1}(\Omega),\left[H_{0}^{-1}(\Omega), L_{2}(\Omega)\right]_{1 / 2,1}\right]_{s, 2} \longrightarrow\left[H^{1}(\Omega), B_{2, \infty}^{3 / 2}(\Omega)\right]_{s, 2}
$$

при $0<s<1$. Здесь пространство справа есть $H^{1+s / 2}(\Omega)$ по формуле (2.8). Пространство слева по теореме реитерации (см. утверждение 2 в предыдущем пункте) есть

$$
\left[H_{0}^{-1}(\Omega), L_{2}(\Omega)\right]_{s / 2,2}=\left[H^{1}(\Omega)^{*}, L_{2}(\Omega)\right]_{s / 2,2},
$$

т.е., в силу утверждения 3 из предыдушего пункта,

$$
\left(\left[H^{1}(\Omega), L_{2}(\Omega)\right]_{s / 2,2}\right)^{*}=H^{1-s / 2}(\Omega)^{*}=H_{0}^{-1+s / 2}(\Omega) .
$$

Заменяя $s / 2$ на $t$, получаем утверждение теоремы 2.1 при $g=0$.

Если теперь $f \in H_{0}^{-1+t}(\Omega)$ и $g \in H^{-1 / 2+t}(\Gamma), 0<t<1 / 2$, то $(f, v)_{\Omega}+\left(g, v^{+}\right)_{\Gamma}$ можно переписать в виде $(\widetilde{f}, v)_{\Omega}$, где снова $\widetilde{f} \in H_{0}^{-1+t}(\Omega)$. Поэтому результат распространяется на $g \neq 0$. Теорема 2.1 доказана.

2.5. Оператор $\mathscr{L}$ и его свойства в липшицевых областях. Теперь мы дополнительно принимаем, что вьполнены условия формальной самосопряженности, и полагаем $g=0$. Определим оператор $\mathscr{L}: u \mapsto f$ формулой (1.29) с $g=0$ :

$$
\Phi(u, v)=(f, v)_{\Omega} .
$$

Предположим (не ограничивая общности), что вьполнено условие (1.28). Тогда этот оператор изоморфно отображает $H^{1}(\Omega)$ на $H_{0}^{-1}(\Omega)$. Это в сушности хорошо известное утверждение (ср. п. 2.2 выше). Но, используя теорему 2.1 , мы можем усилить его. 
Tеорема 2.3. Сужение оператора $\mathscr{L}$ на $H^{t+1}(\Omega)$ устанавливает изоморфизм между этим пространством и пространством $H_{0}^{t-1}(\Omega)$ при $0 \leqslant t<1 / 2$.

ДокАЗАТЕльство. Достаточно рассмотреть случай $0<t<1 / 2$. Проверим ограниченность оператора $\mathscr{L}$. Пусть $u \in H^{t+1}(\Omega)$. Тогда $\partial_{j} u \in H^{t}(\Omega)$. Пусть $v \in$ $H^{1-t}(\Omega)$. Тогда $\partial_{j} v \in H^{-t}(\Omega), v^{+} \in H^{1 / 2-t}(\Gamma)$ и форма $\Phi(u, v)$ сохраняет смысл. Она может быть представлена в виде $(f, v)_{\Omega}$ с $f \in H_{0}^{t-1}(\Omega)$. Таким образом, $\mathscr{L} u=$ $f \in H_{0}^{t-1}(\Omega)$. Легко видеть, что это ограниченный оператор. В силу теоремы 2.1 его образ - все пространство $H_{0}^{t-1}(\Omega)$. А так как сохраняется взаимная однозначность, то остается сослаться на теорему Банаха об ограниченности обратного оператора.

Теперь напомним, что известен также второй способ определения оператора $\mathscr{L}$ (см. [56; гл. VI, §2] или [21; гл. 10]).

ПРЕДЛОЖЕНИЕ 2.4. Существует единственный самосопряженный оператор $\mathscr{L}: u \mapsto f$ в $H^{0}(\Omega)$, определяемый формулой (2.33), с областью определения $\mathscr{D}$, содержащейся в $H^{1}(\Omega)$.

Мы сохранили для этого оператора прежнее обозначение с учетом того, что он совпадает с предыдушим оператором на $\mathscr{D}$. Но иногда их придется различать, тогда первый оператор и его сужения на $H^{1+t}(\Omega)$ будут обозначаться через $\mathscr{L}^{(1)}$, а второй через $\mathscr{L}^{(2)}$.

Очевидно, что оператор $\mathscr{L}^{(2)}$ имеет дискретньй положительньй спектр и что его собственные функции принадлежат, по крайней мере, $H^{1}(\Omega)$. Оператор $\mathscr{L}^{(1)}$ имеет тот же спектр и те же собственные функции.

В случае области с границей класса $C^{1,1}$, как мы видели в п. 1.4, $\mathscr{D}=H^{2}(\Omega, B)$. Аналогичньй результат верен в случае скалярного уравнения с вешественными старшими коэффициентами в вьпуклой области, см. п. 2.8 .

K сожалению, нельзя сказать ничего определенного о $\mathscr{D}$ в общем случае. Но из самосопряженности оператора $\mathscr{L}^{(2)}$ вытекает следующее важное утверждение [56]. Пусть $\mathscr{M}$ - самосопряженный положительньй оператор $\left[\mathscr{L}^{(2)}\right]^{1 / 2}$, квадратньй корень из $\mathscr{L}^{(2)}$.

ПРЕДЛОЖЕНИЕ 2.5. Область определения оператора $\mathscr{M}$ - пространство $H^{1}(\Omega)$, и $\mathscr{M}$ изоморфно отображает это пространство на $H^{0}(\Omega)$.

Теперь докажем следующее предложение, которое ниже будет дополнено в предложении 2.7 и окончательной теореме 2.12 .

ПРЕДЛОЖЕНИЕ 2.6. Пусть $\left\{u_{l}\right\}_{1}^{\infty}$ - ортонормированный базис в $H^{0}(\Omega)$ из собственных функций оператора $\mathscr{L}^{(2)}$. Тогда это безусловный базис во всех пространствах $H^{t}(\Omega)$ и $H_{0}^{-t}(\Omega)$ при $0<t \leqslant 1$.

ДокАЗАТЕЛьСТво. Базисность системы $\left\{u_{l}\right\}$ в $H^{1}(\Omega)$ очевидна. Из предложения 2.5 следует, что степени $\mathscr{M}^{t}$ оператора $\mathscr{M}, 0<t<1$, изоморфно отображают $H^{t}(\Omega)$ на $H^{0}(\Omega)$. Так как $\mathscr{M}^{t}$ переводит в себя функции $u_{l}$ с точностью до множителей, то отсюда получается доказьваемое утверждение для $H^{t}(\Omega)$.

Рассмотрим теперь оператор $\mathscr{N}_{t}$, сопряженньй к $\mathscr{M}^{t}$ как к ограниченному оператору из $H^{t}(\Omega)$ в $H^{0}(\Omega)$. Легко видеть, что $\mathscr{N}_{t}$ изоморфно отображает пространство $H^{0}(\Omega)$ на пространство $\left[H^{t}(\Omega)\right]^{*}=H_{0}^{-t}(\Omega)$. 
Проверим, что оператор $\mathscr{N}_{t}$ переводит функции $u_{l}$ в себя с точностью до множителей. Удобнее рассмотреть для этого обратньй оператор $\mathscr{N}_{t}^{-1}$. Мы имеем

$$
\left(\mathscr{N}_{t}^{-1} u_{l}, u_{k}\right)=\left(u_{l}, \mathscr{M}^{-t} u_{k}\right)=\delta_{k l} \lambda_{l}^{-t / 2} .
$$

Отсюда ясно, что $\mathscr{N}_{t}^{-1} u_{l}=\lambda_{l}^{-t / 2} u_{l}$. Значит, система $\left\{u_{l}\right\}$ остается базисом во всех $H_{0}^{-t}(\Omega), 0 \leqslant t \leqslant 1$.

Кстати, видно также, что $\mathscr{N}_{t}=\mathscr{N}^{t}, 0 \leqslant t \leqslant 1$, где $\mathscr{N}$ - оператор, сопряженньй к оператору $\mathscr{M}: H^{1}(\Omega) \rightarrow H^{0}(\Omega)$.

Следуюшее предложение является основным в этом пункте.

ПРЕДЛОЖЕНИЕ 2.7. Пусть $\Omega$ - липшищева область $u\left\{u_{l}\right\}$ - ортонормированный базис из собственных функиий оператора $\mathscr{L}$ в $H^{0}(\Omega)$. Тогда эта система остается безусловным базисом в $H^{t}(\Omega)$ при $0<t<3 / 2$.

ДокАЗАТЕЛьСтво. Достаточно проверить это при $1<t<3 / 2$. Для этих $t$ положим $\tau=3 / 2-t$. Оператор $\mathscr{N}^{-1 / 2-\tau}$ переводит базис $\left\{u_{l}\right\}$ в $H^{0}(\Omega)$ в базис $\left\{\lambda_{l}^{-1 / 2-\tau} u_{l}\right\}$ в $H_{0}^{-1 / 2-\tau}(\Omega)$. Теперь оператор $\left[\mathscr{L}^{(1)}\right]^{-1}$ переводит этот базис в базис $\left\{\lambda_{l}^{3 / 2-\tau} u_{l}\right\}$ в $H^{3 / 2-\tau}(\Omega)$.

Скалярное произведение в $H^{t}(\Omega), 0<t<3 / 2$, нетрудно определить так, что базис $\left\{u_{l}\right\}$ станет ортогональным.

СлЕДСТВИЕ 2.8. Пусть $1 / 2 \leqslant t<1$. Тогда при $g \in H_{0}^{t-1}(\Gamma)$ ряд вида (8) сходится к следу решения в $H^{t}(\Gamma)$.

Это верно для всех рассматриваемых нами систем.

2.6. Понижение гладкости правых частей и решений. Итак, задача (1.1), $(1.4)$ в липшицевой области $\Omega$ в обобщенной постановке $(1.29)$ имеет одно и только одно решение $u \in H^{1+s}(\Omega)$ при $f \in H_{0}^{-1+s}(\Omega), g \in H^{-1 / 2+s}(\Gamma)$, если $0 \leqslant s<1 / 2, \lambda=0$ (для определенности) и выполнено неравенство (1.28). Для уравнения (2.5) с граничным условием Неймана это по существу результат из [111]. Там же содержится аналогичньй результат для этой задачи при $-1 / 2<s<0$. В отношении доказательства там указано только, что используется техника транспонирования из [74; гл. 2, п. 6.1].

Мы хотим здесь показать, что аналогичный результат верен для рассматриваемых нами систем, но сделаем это несколько более подробно.

Прежде всего обратим внимание на то, что соотношение (1.29) сохраняет смысл при $u \in H^{1 \pm s}(\Omega), v \in H^{1 \mp s}(\Omega), f \in H_{0}^{-1 \pm s}(\Omega), g \in H^{-1 / 2 \pm s}(\Gamma), 0 \leqslant s<1 / 2$. Действительно, в этом случае $\partial_{\nu} u \in H^{ \pm s}(\Omega), \partial_{\nu} v \in H^{\mp s}(\Omega)$, а это взаимно сопряженные пространства. Поэтому форма $\Phi_{0}(u, v)$ сохраняет смысл. В отношении остальных слагаемых в форме $\Phi(u, v)$ не возникает никаких проблем. Очевидно также, что $f$ и $v$ принадлежат взаимно сопряженным пространствам в $\Omega$, а $g$ и $v^{+}-$на $\Gamma$, так что формы $(f, v)_{\Omega}$ и $\left(g, v^{+}\right)_{\Gamma}$ тоже сохраняют смысл. Задачу нахождения $u \in H^{1 \pm s}(\Omega)$ при заданных $f \in H_{0}^{-1 \pm s}(\Omega), g \in H^{-1 / 2 \pm s}(\Gamma)$ можно понимать в смысле справедливости равенства (1.29) при любом $v \in H^{1 \mp s}(\Omega)$, и в случае верхних знаков мы уже знаем, что она однозначно разрешима.

Теперь покажем, что аналогичный результат верен в случае нижних знаков. Предположим сначала, что $g=0$. 
Задаче (1.1), (1.4) с $g=0$ и формально сопряженной задаче

$$
L^{(*)} v=h \quad \text { в } \Omega, \quad B^{(*)} v=0 \text { на } \Gamma
$$

отвечает одна и та же форма $\Phi(u, v)($ см. (1.12)), и мы можем написать

$$
\Phi(u, v)=(f, v)_{\Omega}=(u, h)_{\Omega},
$$

где $u \in H^{1-s}(\Omega), v \in H^{1+s}(\Omega), f \in H_{0}^{-1-s}(\Omega), h \in H_{0}^{-1+s}(\Omega), 0<s<1 / 2$. Задача (2.34) при нашем предположении (1.28) однозначно разрешима, так что $v$ и $h$ связаны изоморфизмом $\mathscr{I} h=v$, переводяшим $H_{0}^{-1+s}(\Omega)$ в $H^{1+s}(\Omega)$. Поэтому $(f, v)_{\Omega}$ можно рассматривать как заданную антилинейную форму на пространстве $H_{0}^{-1+s}(\Omega)$ функций $h$. Так как $u$ - элемент сопряженного пространства $H^{1-s}(\Omega)$, то он однозначно определяется вторым равенством в (2.35). При этом автоматически верно первое равенство (так как соотношение $\Phi(u, v)=(u, h)_{\Omega}$ определяет $v=\mathscr{I} h$ ), так что $u$ - решение задачи (1.1), (1.4).

Пусть теперь граничное условие неоднородно. Тогда функционал $\left(g, v^{+}\right)_{\Gamma}$ с $g \in$ $H^{-1 / 2+s}(\Gamma)$ можно переписать в виде $\left(f_{1}, v\right)_{\Omega}$ с $f_{1} \in H_{0}^{-1+s}(\Omega)$. Заменяя $g$ на 0 и $f$ на $f+f_{1}$, приходим к уже рассмотренной задаче.

Таким образом, окончательно получается следующая теорема.

Теорема 2.9. Пусть $|s|<1 / 2$ и выполнено условие (1.28). Тогда задача (1.1), (1.4) в обобщенной постановке (1.29) при любых $f \in H_{0}^{-1+s}(\Omega), g \in H^{-1 / 2+s}(\Gamma)$ имеет одно и только одно решение $u \in H^{1+s}(\Omega)$. Если $-1 / 2<s<s^{\prime}<1 / 2$ u и решение из $H^{1+s}(\Omega)$, а $f \in H_{0}^{-1+s^{\prime}}(\Omega), g \in H^{-1 / 2+s^{\prime}}(\Gamma)$, mо $u \in H^{1+s^{\prime}}(\Omega)$.

ЗАмечАниЕ 2.10. Для скалярного уравнения (1.22) с граничным условием Неймана такая теорема доказана также в [91], [92] при помощи потенциалов, причем там рассмотрены значительно более общие пространства. Однако, насколько известно автору, пока теорема 2.9 применима к намного более общим системам, чем метод потенциалов. Это будет объяснено в $\S 5$.

ЗАмечАНИЕ 2.11. Отметим следствие из теоремы 2.9, которое можно отнести к теории соболевских пространств в липшищевых областях. Форма $\Phi(u, v)$, удовлетворяющая условию (1.28), определяет двойственность между пространствами $H^{1+s}(\Omega)$ и $H^{1-s}(\Omega),|s|<1 / 2$, и они оказываются рефлексивными относительно этой формы.

Теперь сформулируем наше окончательное утверждение о базисности.

ТЕОРема 2.12. Пусть $\Omega$ - липиицева область $и\left\{u_{l}\right\}$ - ортонормированный базис из собственных функиий оператора $\mathscr{L}$ в $H^{0}(\Omega)$. Тогда эта система остается безусловным базисом в пространствах $H^{t}(\Omega)$ и $H_{0}^{-t}(\Omega)$ при $0<t<3 / 2$.

Действительно, с учетом предложений 2.6 и 2.7 нам остается проверить это только для $H_{0}^{-t}(\Omega)$ при $1<t<3 / 2$. Для этого достаточно воспользоваться уже известньм результатом для $H^{s}(\Omega), s=2-t$, и соответствуюшим изоморфизмом, см. теоремy 2.9 .

Напомним, что при $t \geqslant 3 / 2$ не только базисности, но и полноты в $H^{t}(\Omega)$ нет даже для областей с гладкой гранищей. 
2.7. Скалярное уравнение с вещественными старшими коэффициентами в выпуклой области. Рассмотрим скалярную задачу

$$
\begin{gathered}
-\operatorname{div} A(x) \operatorname{grad} u(x)+\sum b_{j} \partial_{j} u(x)+c(x) u(x)=f(x) \quad \text { в } \Omega, \\
\partial_{\nu_{a}} u(x)=\mu u(x) \text { на } \Gamma
\end{gathered}
$$

с вещественной симметричной положительно определенной матрицей $A(x)$. Область $\Omega$ предполагается вьпуклой, в частности, липшищевой. Следующая теорема принадлежит Гривару, см. [44]. См. также более ранние результаты Ладыженской и Уральцевой в $[71 ;$ гл. III, $\S 8]$.

Теорема 2.13. При $\mu \leqslant 0, f \in H^{0}(\Omega)$ решение задачи (2.36) в обобщенной постановке (2.33) принадлежит $H^{2}(\Omega)$.

У Гривара охвачены также случаи нелинейного граничного условия и условия Дирихле. Младших членов в уравнении у него нет, но это несушественно: их можно перенести к правой части уравнения, получится функция из $L_{2}(\Omega)$. Мы наметим рассуждение Гривара с небольшим упрощением.

Не ограничивая обшности, заменим младшие члены в уравнении на слагаемое $\alpha u$ c достаточно большим $\alpha>0$ и предположим функции $u$ и $f$ вешественными. Положим $L_{\alpha}=L_{0}+\alpha I$. Ключевым местом является доказательство следующей априорной оценки: для аппроксимируюших $\Omega$ выпуклых областей $\Omega_{\gamma}$ с границами класса $C^{2}$ на функциях из $C^{2}\left(\bar{\Omega}_{\gamma}\right)$ (такие области существуют, см. [44])

$$
\|u\|_{2, \Omega_{\gamma}} \leqslant C_{1}\left\|L_{\alpha} u\right\|_{0, \Omega_{\gamma}} \quad\left(\alpha \geqslant \alpha_{0}\right)
$$

с постоянньми $C_{1}$ и $\alpha_{0}$, не зависяшими от $\gamma$. Имея такую оценку, можно при помощи аппроксимаций показать, что в $\Omega$ сушествует решение из $H^{2}(\Omega)$, и тогда обобшенное решение с ним совпадает.

Для упрошения обозначений вместо $\Omega_{\gamma}$ будем писать $\Omega$, но это сейчас выпуклая область с границей класса $C^{2}$.

Сначала рассмотрим случай матрицы $A$, не зависящей от $x$. При помощи поворота осей координат и растяжения осей она приводится к единичной матрице, т.е. рассматриваемьй оператор приводится к уравнению Гельмгольца. Несложно проверяется следующее упрощающее доказательство соображение: в точках границы конормальная производная переходит при этом в обычную производную вдоль нормали. Таким образом, мы теперь рассматриваем задачу

$$
-\Delta u+\alpha u=f \text { в } \Omega, \quad \partial_{\nu} u^{+}=\mu u^{+} \text {на } \Gamma .
$$

Как показано в [44], для вектор-функций $v(x)$ (размерности $n$ ) из $H^{1}(\Omega)$ справедливо тождество

$$
\begin{aligned}
& \int_{\Omega}|\operatorname{div} v|^{2} d x-\sum_{i, j} \int_{\Omega} \partial_{i} v_{j} \cdot \partial_{j} v_{i} d x \\
& =-2\left(v_{\tau}^{+}, \operatorname{grad}_{\tau}\left(v_{\nu}^{+}\right)\right)_{\Gamma}-\int_{\Gamma}\left[\mathscr{B}\left(v_{\tau}^{+}, v_{\tau}^{+}\right)+\operatorname{tr} \mathscr{B}\left(v_{\nu}^{+}\right)^{2}\right] d S
\end{aligned}
$$


Здесь $v_{\tau}^{+}$и $v_{\nu}^{+}$- касательная и нормальная составляющие вектора $v$ на гранище, $\operatorname{grad}_{\tau}$ - касательная составляющая градиента, $\mathscr{B}$ - некоторая квадратичная форма, неположительно определенная в силу вьпуклости границы. Положим, как в [44], $v=\operatorname{grad} u$, $u \in C^{2}(\bar{\Omega})$. Тогда $v_{\nu}^{+}=\partial_{\nu_{a}} u^{+}=\mu u^{+}$и первьй член справа в (2.39) принимает вид $-\mu\left(\operatorname{grad}_{\tau} u^{+}, \operatorname{grad}_{\tau} u^{+}\right)_{\Gamma}$. Таким образом, вся правая часть тождества $(2.39)$ неотрицательна и получается неравенство

$$
\int_{\Omega}|\Delta u|^{2} d x \geqslant \sum \int_{\Omega} \partial_{i} v_{j} \cdot \partial_{j} v_{i} d x=\sum \int\left|\partial_{i j}^{2} u\right|^{2} d x
$$

В исходных координатах получается (2.37).

Если теперь элементы матрищы $A(x)$ переменные, то после их замораживания в любой точке получается тот же результат с постоянными $C_{1}$ и $\alpha_{0}$, не зависяшими от этой точки. Далее обычная в теории эллиптических задач процедура с использованием достаточно мелкого разбиения единицы и локального перехода от постоянных коэффициентов к почти постоянным приводит к нужному результату.

Относительно возможностей дальнейших обобщений см. [90] и указанную там литературу.

СлЕДСТВИЕ 2.14. В случае скалярного уравнения с вещественными стариими коэффициентами в выпуклой области для оператора $\mathscr{L}$ справедливо утверждение теоремы 1.9.

2.8. Слабые возмущения самосопряженных операторов и асимптотика спектра. Остановимся теперь на асимптотике собственных значений оператора $\mathscr{L}$ в липшицевой области. Для самосопряженных задач Дирихле и Неймана, как показано в [19], заведомо справедливо соотношение (1.30). В [84] получены более сильные оценки остатка для скалярных уравнений. Дальнейшие продвижения получены в работе [93]. Мы сформулируем эти результаты немного дальше.

Сейчас мы поясним, почему асимптотика собственных значений с оптимальной или менее сильной оценкой остатка, установленная для однородной системы (без младших членов) и граничного условия Неймана, влечет такую же оценку для системы с младшими членами и нашего граничного условия $B u=0$ (тоже с младшими членами).

Пусть $\mathfrak{H}$ - комплексное сепарабельное гильбертово пространство с нормой $\|\cdot\|$ и $\mathfrak{A}_{0}-$ неограниченный замкнутый оператор в $\mathfrak{H}$ с плотной областью определения. Пусть $\mathfrak{A}_{0}$ обратим, и пусть $\mathfrak{A}_{1}-$ второй оператор с областью определения, содержащей область определения оператора $\mathfrak{A}_{0}$. Предположим, что он компактен относительно $\mathfrak{A}_{0}$, т.е. оператор $\mathfrak{A}_{1} \mathfrak{A}_{0}^{-1}$ компактен. Тогда оператор $\mathfrak{A}=\mathfrak{A}_{0}+\mathfrak{A}_{1}$ называют слабым возмущением оператора $\mathfrak{A}_{0}$.

Предположим (этого достаточно для нас), что $\mathfrak{A}_{0}-$ полуограниченньй, для определенности снизу, оператор с дискретным спектром. Условимся нумеровать его собственные значения в порядке неубывания с учетом кратностей. (Если оператор полуограничен сверху, собственные значения нумеруются в порядке невозрастания.)

Следующее утверждение принадлежит Келдышу; его доказательство содержится в $[41 ;$ гл. V, $\S 11]$. 
ПРЕДЛОЖЕНИЕ 2.15. Если собственные значения оператора $\mathfrak{A}_{0}$ имеют асимптотику

$$
\lambda_{j}=c j^{p}+o\left(j^{p}\right)
$$

где с и ляется слабым возмущением оператора $\mathfrak{A}_{0}$, то $\mathfrak{A}$ тожсе имеет дискретныи спектр и полуограничен снизу, а его собственнье значения имеют ту же асимптотику.

Далее в этом пункте мы рассматриваем “более слабые" возмущения и формулируем результаты о сохранении асимптотики с улучшенной оценкой остатка.

Функцией распределения неубывающей последовательности $\left\{\lambda_{j}\right\}$ назьвают функцию $N(t)$, равную при каждом $t$ числу таких $j$, что $\lambda_{j}<t$. Если это функция распределения собственных значений оператора $\mathfrak{A}$, то будем писать $N_{\mathfrak{A}}(t)$.

Асимптотическую формулу для собственных значений оператора $\mathfrak{A}$, если она имеется, обьчно можно эквивалентным образом переписать в виде асимптотической формулы для их функции распределения (и наоборот). Например, формула (1.25), как несложно проверить, эквивалентна формуле вида

$$
N_{\mathscr{L}}(t)=\widetilde{C}^{(n)} t^{n / 2}+O\left(t^{(n-1) / 2}\right)
$$

а формула (1.30) эквивалентна аналогичной формуле с оценкой остатка $o\left(t^{n / 2}\right)$. Отметим, кстати, что постоянная $\widetilde{C}^{(n)}$ выражается через главный символ системы следуюшим образом:

$$
\widetilde{C}^{(n)}=(2 \pi)^{-n} \int_{\Omega} \int_{\mathbb{R}^{n}} N_{a}(1) d \xi d x
$$

где $N_{a}(t)$ - функция распределения собственных значений главного символа $a(x, \xi)$ (см., например, [109; п. 9.2]).

В упомянутых в начале пункта результатах из [84] оценка остатка в (2.42), в частности, в случае самосопряженных скалярных задач Дирихле или Неймана имеет вид $O\left(t^{(n-1 / 2) / 2}\right)$ в общем случае и $O\left(t^{(n-1) / 2} \ln t\right)$ в случае постоянных коэффициентов. В [93] последняя оценка получена в предположении, что старшие коэффициенты уравнения второго порядка вешественны и обладают некоторой добавочной гладкостью.

Пусть $q<1$. Оператор $\mathfrak{A}_{1}$ назовем $q$-подчиненнылм оператору $\mathfrak{A}_{0}$, если $\mathfrak{A}_{1}=\mathfrak{B} \mathfrak{A}_{0}^{q}$, где $\mathfrak{B}$ - ограниченньй оператор в $\mathfrak{H}$. Более общее определение со ссылками на литературу см. в [78], [79] или [76; гл. I, $§ 5]$.

В [79] (см. также [76; гл. I, §8]) получен следуюший результат.

ПреДЛОжЕнИЕ 2.16 (Маркус, Мацаев). Пусть оператор $\mathfrak{A}_{1}$ q-подчинен оператору $\mathfrak{A}_{0}$ при некотором $q, 0 \leqslant q<1$, и пусть $\mathfrak{A}$ - самосопряжсенный оператор $\mathfrak{A}_{0}+\mathfrak{A}_{1}$. Тогда

$$
\left|N_{\mathfrak{A}}(t)-N_{\mathfrak{A}_{0}}(t)\right| \leqslant C_{1}\left[N_{\mathfrak{A}_{0}}\left(t+b t^{q}\right)-N_{\mathfrak{A}_{0}}\left(t-b t^{q}\right)\right], \quad t \geqslant t_{0},
$$

при достаточно больиих положстельных постоянных $C_{1}, b$ и $t_{0}$.

Отсюда для оператора $\mathscr{L}$ из п. 1.3 вьводится следующее утверждение, которое было нужно в п. 1.6 с $q=1 / 2$. 
ПРЕДЛОЖЕНИЕ 2.17. При добавлении к оператору $\mathscr{L}$ из $n .1 .3$ q-подчиненного ему оператора получается оператор с такой жсе асимптотикой собственньх значений, как в (1.25), если $q \leqslant 1 / 2$.

При этом сохраняется постоянная $C^{(n)}$ в главном члене асимптотики. Если предположить, что для остаточного члена справедлива более слабая оценка, то она также сохраняется.

Пусть $\Psi_{0}(\mathfrak{u}, \mathfrak{v})$ и $\psi(\mathfrak{u}, \mathfrak{v})$ - две эрмитовы формы на $\mathfrak{H}$, область определения $\mathscr{D}\left(\Psi_{0}\right)$ первой формы содержится в области определения $D(\psi)$ второй формы, и пусть форма $\Psi_{0}(\mathfrak{u}, \mathfrak{u})$ положительна при $\mathfrak{u} \neq 0$. Форма $\psi$ назьвается $q$-подчиненной форме $\Psi_{0}$, $0 \leqslant q<1$, если

$$
|\psi(\mathfrak{u}, \mathfrak{u})| \leqslant C_{2} \Psi_{0}(\mathfrak{u}, \mathfrak{u})^{q}\|\mathfrak{u}\|^{2-2 q}
$$

на $\mathscr{D}\left(\Psi_{0}\right)$. Сp. [77], [82], [7] и [54].

Заметим, что если $\mathfrak{A}_{0}$ - самосопряженньй положительньй оператор с дискретньм спектром $\left\{\lambda_{j}\right\}$ и самосопряженный оператор $\mathfrak{A}_{1} q$-подчинен оператору $\mathfrak{A}_{0}, 0 \leqslant q<1$, то форма $\left(\mathfrak{A}_{1} \mathfrak{u}, \mathfrak{v}\right)$ оператора $\mathfrak{A}_{1} q$-подчинена форме $\left(\mathfrak{A}_{0} \mathfrak{u}, \mathfrak{v}\right)=\left(\mathfrak{A}_{0}^{1 / 2} \mathfrak{u}, \mathfrak{A}_{0}^{1 / 2} \mathfrak{v}\right)$ оператора $\mathfrak{A}_{0}$. Это легко проверяется при помоши разложения вектора $\mathfrak{u}$ по собственным векторам оператора $\mathfrak{A}_{0}$ и неравенства Гёльдера: если $c_{j}$ - соответствуюшие коэффишиенты Фурье, то

$$
\left|\left(\mathfrak{B A}_{0}^{q} \mathfrak{u}, \mathfrak{u}\right)\right| \leqslant C_{3}\left(\mathfrak{A}_{0}^{q} \mathfrak{u}, \mathfrak{u}\right)=C_{3} \sum \lambda_{j}^{q}\left|c_{j}\right|^{2} \leqslant C_{3}\left(\sum \lambda_{j}\left|c_{j}\right|^{2}\right)^{q}\left(\sum\left|c_{j}\right|^{2}\right)^{1-q}
$$

Подробнее о связи форм с операторами см. в [56] и [19].

Пусть теперь форма $\Psi_{0}(\mathfrak{u}, \mathfrak{v})$ определяет самосопряженный оператор $\mathfrak{A}_{0}$ с дискретным положительным спектром, а форма $\Psi(\mathfrak{u}, \mathfrak{v})=\Psi_{0}(\mathfrak{u}, \mathfrak{v})+\psi(\mathfrak{u}, \mathfrak{v})$ определяет самосопряженный оператор $\mathfrak{A}$. Эти два оператора уже не обязательно имеют одинаковые области определения.

В [77] получен, в частности, следуюший аналог предложения 2.16 для этого случая.

ПРЕДЛОЖЕнИЕ 2.18 (Маркус, Мацаев). Пусть форма $\psi$ q-подчинена форме $\Psi_{0}$ при некотором $q, 0 \leqslant q<1$. Тогда $\mathfrak{A}-$ полуограниченный снизу оператор $c$ дискретным спектром и справедливо соотношение вида (2.44).

Отсюда выводится

ПРЕДЛОЖЕНИЕ 2.19. При добавлении к форме $\Phi_{0}$ любой q-подчиненной формь оченка остатка в формуле (1.25) для собственных значений соответствующего оператора ( или аналогичной формуле с более слабой оченкой остатка) заведомо не ухудиается при $q \leqslant 1 / 2$.

Вернемся к формам из п. 1.1. Из (1.17)-(1.19) видно, что форма $\Phi(u, v)-\Phi_{0}(u, v)$ $1 / 2$-подчинена форме $\Phi_{0}(u, u)$. 
ЗАмЕчАнИЕ 2.20. В литературе имеется ряд работ о спектральной асимптотике для уравнений с негладкими коэффициентами. Этот направление непосредственно не относится к теме нашей статьи, но используемые в этих работах подходы могут оказаться полезными при рассмотрении негладких границ. Потому мы указьваем две недавние работы [50] и [128], в которых можно найти дальнейшие ссылки. Некоторые новые работы Иврия и Зелиньского находятся в печати.

2.9. Системы с особенностями в младшем члене. Пусть в обозначениях п. 1.6 в форму $\Phi(u, v)$ добавлено слагаемое $\left(c_{2} u, v\right)_{\Omega}$. Как легко видеть, оно $1 / 2$-подчинено остальной части формы $\Phi(u, v)$. Это позволяет, сдвинув, если надо, спектральный параметр, снова определить операторы $\mathscr{L}^{(2)}$ и $\mathscr{L}^{(1)}$. Оператор $\mathscr{L}^{(2)}$ остается самосопряженньм и имеет положительный дискретный спектр. Асимптотика его собственных значений сохраняется (см. предложение 2.19). Теорема 2.1 тоже сохраняется, так как слагаемое $c_{2} u$ можно перенести к правой части уравнения.

В отношении действия оператора умножения на матрищу $c_{2}$ на функщии меньшей гладкости, чем в $H^{1}(\Omega)$, отметим следующее. Мы считаем эту матрицу эрмитовой. Из того, что это мультипликатор из $H^{1}(\Omega)$ в $H^{0}(\Omega)$, следует, что это мультипликатор из $H^{0}(\Omega)$ в $H_{0}^{-1}(\Omega)$. Используя интерполящию, получаем, что это мультипликатор из $H^{\theta}(\Omega)$ в $H_{0}^{\theta-1}(\Omega)$ при $0 \leqslant \theta \leqslant 1$. Ср. $[80 ; \S 2.2$ и $\S 2.4]$.

Из сказанного следует, что остаются в силе все дальнейшие результаты пп. 2.5 и 2.6 .

\section{§ 3. Варианты и обобщения}

3.1. Задача Дирихле. Для этой задачи

$$
\mathscr{L} u=\lambda u+f \text { в } \Omega, \quad u^{+}=h \text { на } \Gamma
$$

мы сформулируем аналоги нескольких основных утверждений из предыдущих параграфов, но проверять все заново не будем, поскольку доказательства в основном сохраняются. В условии II нет нужды, мы предполагаем только, что система сильно эллиптична.

1. Исходная теорема о разрешимости в областях с гладкой границей уже сформулирована в п. 1.1 (теорема 1.1), там же отмечена коэрцитивность формы $\Phi(u, v)$ на пространстве $H_{0}^{1}(\Omega)$. Задача Дирихле для сильно эллиптической системы, как известно, эллиптична (ср. [103]). Обозначим через $H^{t}(\Omega, D)$ подпространство в $H^{t}(\Omega)$, составленное из функций, удовлетворяюших однородному условию Дирихле $u^{+}=0$ $(t>1 / 2)$. В отличие от граничного условия $B u=0$ оно при $t \leqslant 2$ “держится" для $t>1 / 2$. Последнее подпространство совпадает с $H_{0}^{t}(\Omega)$ при $1 / 2<t<3 / 2$. Аналог теоремы 1.7 получается следуюший:

ТЕОРема 3.1. При бесконечной гладкости границы и коэффициентов формально самосопряженной задаче $(3.1)$ с $h=0$ отвечает оператор $\mathscr{L}_{D}$ в $H^{0}(\Omega)$ с областью определения $H^{2}(\Omega, D)$. Это самосопряженный полуограниченный снизу оператор с дискретным спектром и собственными значениями, имеющими асимптотику (1.25). Собственные функиии принадлежат $C^{\infty}(\bar{\Omega})$. Составленный из них ортонормированный базис в $H^{0}(\Omega)$ остается безусловным базисом в $H^{t}(\Omega)$ при $0 \leqslant t<1 / 2$ и в $H^{t}(\Omega, D)$ при $1 / 2<t \leqslant 2$. 
Переход на гранищу в разложениях по собственньм функциям со спектральным параметром $\lambda$, конечно, не интересен, поскольку собственные функции имеют нулевые граничные значения. Другими словами, рассматриваемая сейчас спектральная задача не имеет отношения к первому варианту метода $R$-матрицы. Но результаты, приведенные в настоящем пункте, будут существенно использованы в главе II.

При предположениях п. 1.5 (граница класса $C^{1,1}$ и пониженные предположения о гладкости коэффициентов) собственные функции принадлежат $H^{2}(\Omega)$ [44], [83], а только что приведенное утверждение о базисности сохраняется. Обобщенную постановку задачи мы сейчас уточним.

2. Пусть теперь $\Omega$ - липшицева область. Форма $\Phi(u, v)$, отвечаюшая задаче Дирихле, определяется формулой (1.12) с $b=0$. Обобщенная постановка задачи при $u^{+}=h=0$ и $\lambda=0$ имеет вид

$$
\Phi(u, v)=(f, v)_{\Omega}, \quad v \in H_{0}^{1}(\Omega)
$$

Здесь $f \in H^{-1}(\Omega)$ и решение ишется в $H_{0}^{1}(\Omega)$. Но можно предположить, что $f \in$ $H^{-1+t}(\Omega), v \in H_{0}^{1-t}(\Omega)$, и искать решение в $H_{0}^{1+t}(\Omega)$ при $|t|<1 / 2$. Если граничное условие неоднородное и $h \in H^{1 / 2+t}(\Gamma)$, то задача сводится к задаче с однородным граничным условием стандартным способом - вычитанием из решения функции $u_{0} \in$ $H^{1+t}(\Omega)$ с $u_{0}^{+}=h$; при этом $f$ заменяется новой функцией из $H^{-1+t}(\Omega)$.

Сформулируем аналог теоремы 2.9 .

Теорема 3.2. Пусть форма $\Phi$, отвечающая задаче Дирихле, удовлетворяет условию (1.28) на $H_{0}^{1}(\Omega)$. Тогда при $\lambda=0$, если $f \in H^{-1+t}(\Omega), h \in H^{1 / 2+t}(\Gamma)$ u $|t|<1 / 2$, существует и единственно решение $u \in H^{1+t}(\Omega)$.

Эта теорема доказана в [111] для скалярного уравнения (2.5) с вещественнњми коэффициентами и переносится на рассматриваемые нами сильно эллиптические системы (1.1). В [91], [92] теорема 3.2 доказана методом потенциалов (см. ниже $§ 5$ ), и там рассмотрены более общие пространства. Для уравнения Пуассона $\Delta u=f$ она содержится в [53], и там же приведен пример, показьвающий, что при $t=1 / 2$ ее утверждение неверно. При $t=-1 / 2$ оно неверно даже для областей с гладкой границей. См. ниже замечание 5.7 .

Рассмотрим теперь оператор $\mathscr{L}_{D}$, отвечающий формально самосопряженной задаче Дирихле с однородным граничным условием. Как и в случае граничного условия $B u=0$, операторов два. Первьй оператор изоморфно отображает пространство $H_{0}^{1+t}(\Omega)$ на $H^{-1+t}(\Omega)$ при $0 \leqslant t<1 / 2$. Второй определен как самосопряженньй оператор в $H^{0}(\Omega)$ с областью определения, содержашейся в $H_{0}^{1}(\Omega)$. Оба имеют один и тот же дискретньй спектр и одни и те же собственные функции. Квадратньй корень из второго оператора изоморфно отображает $H_{0}^{1}(\Omega)$ на $H^{0}(\Omega)$. Такие же рассмотрения, как в пп. 2.5-2.6, приводят к следуюшему аналогу теоремы 2.12 .

ТЕОРема 3.3. Ортонормированный базис в $H^{0}(\Omega)$ из собственных функиий оператора $\mathscr{L}_{D}$ остается безусловным базисом в $H^{t}(\Omega)$ при $-1 / 2<t<1 / 2$, а также в $H_{0}^{t}(\Omega)$ и $H^{-t}(\Omega)$ nри $1 / 2<t<3 / 2$.

Что касается поведения собственных значений, то они заведомо имеют асимптотику (1.30) [19], а об оценке остатка можно сказать то же самое, что и в п. 2.8. 
3.2. Несколько определений и утверждений из теории линейных операторов. Этот пункт содержит определения и формулировки результатов, которые понадобятся нам для перехода к задачам, близким к самосопряженным (в частности, в следующем пункте).

Через $\mathfrak{H}$ снова обозначаем комплексное сепарабельное гильбертово пространство со скалярным произведением $(\cdot, \cdot)$ и нормой $\|\cdot\|$.

1. Пусть $\mathfrak{T}$ - линейный ограниченный оператор в $\mathfrak{H}$ и $\mathfrak{T}^{*}-$ сопряженный к $\mathfrak{T}$ оператор. Действительная и мнимая части оператора $\mathfrak{T}$ определяются равенствами

$$
\operatorname{Re} \mathfrak{T}=\frac{1}{2}\left(\mathfrak{T}+\mathfrak{T}^{*}\right) \quad \text { и } \quad \operatorname{Im} \mathfrak{T}=\frac{1}{2 i}\left(\mathfrak{T}-\mathfrak{T}^{*}\right) .
$$

Очевидно, что это самосопряженные операторы и что $\mathfrak{T}=\operatorname{Re} \mathfrak{T}+i \operatorname{Im} \mathfrak{T}$.

Оператор $\mathfrak{T}$ назьвается диссипативным, если $\operatorname{Im} \mathfrak{T} \geqslant 0$, т.е. $\operatorname{Im}(\mathfrak{T} \mathfrak{u}, \mathfrak{u}) \geqslant 0$ для всех $\mathfrak{u} \in \mathfrak{H}$. Собственные значения такого оператора имеют неотрицательные мнимые части, а в корневых подпространствах, отвечающих вещественньм собственным значениям, нет присоединенных функций (см. [41; гл. V, 1$]$ ).

Характеристические числа $\lambda_{j}$ компактного оператора $\mathfrak{T}$ - это величины, обратные к его ненулевым собственным значениям. В интересующих нас случаях $\operatorname{Re} \lambda_{j}$ будут стремиться $\mathrm{x}+\infty$ (или $\mathrm{\kappa}-\infty)$; уславливаемся, что тогда $\lambda_{j}$ нумеруются, начиная с номера 1 , в порядке неубьвания (соответственно невозрастания) $\operatorname{Re} \lambda_{j}$ с учетом кратностей.

2. Система $\left\{\mathfrak{u}_{j}\right\}$ векторов назьвается полной в $\mathfrak{H}$, если их конечные линейные комбинации всюду плотны в $\mathfrak{H}$.

У изучаемых в дальнейшем компактных операторов нуль может быть собственным значением только конечной кратности, а присоединенных векторов, отвечающих этому собственному значению, как правило нет в силу диссипативности этих операторов.

Если корневые векторы $\mathfrak{u}_{j}$ такого оператора $\mathfrak{T}$ обладают свойством полноты, то строится минимальная полная система из этих векторов и удобно считать, что $\mathfrak{u}_{j}$ с $j \leqslant 0$ отвечают нулевому собственному значению (если оно есть), а $\mathfrak{u}_{j}$ с $j>0$ - характеристическим числам $\lambda_{j}$.

Если $\left\{\mathfrak{u}_{k}\right\}$ - полная минимальная система, то существует биортогональная к ней система $\left\{\mathfrak{v}_{j}\right\}$ и любому вектору $\mathfrak{f} \in \mathfrak{H}$ сопоставляется формальньй meмe $\left\{\mathfrak{u}_{k}\right\}$ :

$$
\mathfrak{f} \sim \sum c_{k} \mathfrak{u}_{k}, \quad \text { где } c_{k}=\left(\mathfrak{f}, \mathfrak{v}_{k}\right)
$$

В интересующем нас случае корневых векторов компактного оператора биортогональная система строится из корневых векторов сопряженного оператора. См., например, $[1 ; \S 36]$ или $[14 ;$ п. 5.2].

Минимальная полная система $\left\{\mathfrak{v}_{k}\right\}$ назьвается базисом в $\mathfrak{H}$, если ряд (3.4) всегда сходится к f. Если, кроме того, сходимость сохраняется при любой перестановке членов, то базис назьвается безусловным, это уже отмечалось в п. 1.3. При $0<C_{1} \leqslant$ $\left\|\mathfrak{u}_{k}\right\| \leqslant C_{2}$ (система в этом случае назьвается почти нормированной) эквивалентное условие состоит в том, что $\left\{\mathfrak{u}_{k}\right\}-6 а з и с$ Puсcа в $\mathfrak{H}$ : это значит, что сушествует ограниченный обратимьй оператор в $\mathfrak{H}$, переводяший эту систему в ортонормированньцй базис. См. [41; гл. VI, §2]. 
Если известно лишш, что в ряде (3.4) можно расставить скобки не зависящим от $\mathfrak{f}$ способом так, что ряд из выражений в скобках всегда сходится к $\mathfrak{f}$ в $\mathfrak{H}$, то система называется базисом со скобками в $\mathfrak{H}$. Если при этом в ряде из выражений в скобках возможны любые перестановки, то эта система - безусловный базис со скобками. См. [76; гл. I, §6].

Менее сильное свойство системы, чем базисность со скобками, но более сильное, чем полнота, состоит в том, что этот ряд является базисом для метода суммирования Абеля-Лидского (со скобками) порядка $r>0$. Поясним, что это такое, предположив сначала для простоты, что присоединенных функций нет и характеристические числа находятся в узком угле с биссектрисой $\mathbb{R}_{+}$. В $k$-й член ряда $(3.4)$ при $k>0$ вставляется множитель $\exp \left(-\lambda_{k}^{r} t\right)$, и предполагается, что это обеспечивает сходимость ряда в $\mathfrak{H}$ после не зависяшей от $\mathfrak{f}$ расстановки скобок при $t>0$; далее, предполагается, что сумма $\mathfrak{f}(t)$ этого ряда стремится к $\mathfrak{f}$ в $\mathfrak{H}$ при $t \downarrow 0$.

Более полное определение метода Абеля-Лидского состоит в следующем. Характеристические числа $\lambda_{j}$ разбиваются (не зависящим от $\mathfrak{f}$ способом) на групшы, которые окружаются простыми замкнутьми гладкими контурами $\gamma_{l}(l=1,2, \ldots)$. Замкнутые ограниченные области с контурами $\gamma_{l}$ попарно не имеют общих точек и уходят на бесконечность при росте $l$. Начиная с некоторого номера $l_{0}$, группа членов ряда (3.4), отвечающая собственным значениям, лежащим внутри $\gamma_{l}$, заменяется интегралом

$$
\left(P_{l r} \mathfrak{f}\right)(t)=\frac{1}{2 \pi i} \int_{\gamma_{l}} \mathfrak{T}(I-\lambda \mathfrak{T})^{-1} \exp \left(-\lambda^{r} t\right) d t \mathfrak{f} .
$$

Групшы с номерами $l<l_{0}$ ничем не заменяются, но мы их будем записьвать тоже в виде $\left(P_{l r} \mathfrak{f}\right)(t)$. Требуется, чтобы ряд $\sum_{l}\left(P_{l r} \mathfrak{f}\right)(t)$ сходился в $\mathfrak{H}$ при $t>0$ и чтобы его сумма $\mathfrak{f}(t)$ стремилась к $\mathfrak{f}$ при $t \downarrow 0$. См. детали в работе Лидского [72], где этот метод назван методом Абеля, а также в $[1 ; \S 35]$ или $[14 ;$ п. 5.6].

ЗАмЕчАНИЕ 3.4. Предположим, что имеется второе гильбертово пространство $\mathfrak{H}_{1}$, компактно вложенное в $\mathfrak{H}$, и что оператор $\mathfrak{T}$ осушествляет изоморфное отображение пространства $\mathfrak{H}$ на $\mathfrak{H}_{1}$. В частности, норма вектора $\mathfrak{T} \mathfrak{f}$ в $\mathfrak{H}_{1}$ эквивалентна норме вектора $\mathfrak{f}$ в $\mathfrak{H}$. Тогда из базисности системы $\left\{\mathfrak{u}_{k}\right\}$ для метода Абеля - Лидского порядка $r>0$ в $\mathfrak{H}$ следует то же самое в $\mathfrak{H}_{1}$ (и наоборот).

Действительно, это вытекает из перестановочности оператора $\mathfrak{T}$ с операторами (3.5). Умножая ряд из выражений (3.5) слева на $\mathfrak{T}$, получаем такой же ряд для $\mathfrak{T} \mathfrak{f}$, которьй сходится при $t>0$ в $\mathfrak{H}_{1}$. Его сумма стремится к $\mathfrak{T} \mathfrak{f}$ при $t \downarrow 0$. То и другое следует из нашего предположения об изоморфизме.

Приведенные определения и только что сделанное замечание естественным образом переносятся на неограниченные операторы $\mathfrak{A}$ с компактной резольвентой $(\mathfrak{A}-\lambda I)^{-1}$, например, на операторы, имеющие компактные обратные операторы $\mathfrak{A}^{-1}=\mathfrak{T}$. Такой оператор $\mathfrak{A}$ имеет дискретный спектр, и через $\lambda_{j}$ мы обозначаем его собственные значения. Их действительные части $\operatorname{Re} \lambda_{j}$ в интересуюших нас случаях будут стремиться к $+\infty$ (или к $-\infty$ ), и тогда мы нумеруем собственные значения в порядке неубьвания (соответственно невозрастания) $\operatorname{Re} \lambda_{j}$ с учетом кратностей.

3. В п. 2.8 мы привели несколько утверждений о сохранении асимптотики спектра при переходе от самосопряженного полуограниченного оператора $\mathfrak{A}_{0}$ с дискретньм 
спектром к его слабому возмущению $\mathfrak{A}=\mathfrak{A}_{0}+\mathfrak{A}_{1}$. В общем случае оператор $\mathfrak{A}$ не обязательно самосопряженньй. Мы хотим теперь отметить, что в этом случае сохраняются все определения и утверждения из этого пункта, если только под $N_{\mathfrak{A}}(t)$ понимать функцию распределения действительных частей собственных значений оператора $\mathfrak{A}$. Ссылки на литературу остаются теми же.

Поясним, что если оператор $\mathfrak{A}_{1}$ компактен относительно оператора $\mathfrak{A}_{0}$, то спектр оператора $\mathfrak{A}$ лежит в объединении сколь угодно узкого угла с биссектрисой $\mathbb{R}_{+}$и круга с центром в начале (зависяшего от выбора этого угла). В случае же, когда $\mathfrak{A}_{1}$ $q$-подчинен оператору $\mathfrak{A}_{0}$, спектр оператора $\mathfrak{A}=\mathfrak{A}_{0}+\mathfrak{A}_{1}$ лежит в объединении окрестности луча $\mathbb{R}_{+}$вида

$$
\left\{\lambda: \operatorname{Re} \lambda>0, \operatorname{Im} \lambda \leqslant C_{1} \operatorname{Re} \lambda^{q}\right\}
$$

и круга с центром в начале. Аналогично обстоит дело, если операторы $\mathfrak{A}_{0}$ и $\mathfrak{A}$ определяются формами и разность этих форм $q$-подчинена форме, определяющей оператор $\mathfrak{A}_{0}$.

4. Сформулируем теперь признаки базисности со скобками и базисности АбеляЛидского системы корневых функций слабых возмушений самосопряженных операторов с дискретным спектром.

ПРЕДЛОЖЕНИЕ 3.5. Пусть собственнье значения $\lambda_{j}$ оператора $\mathfrak{A}_{0}$ имеют асимптотику вида (2.41) или хотя бы удовлетворяют неравенствам

$$
0<C_{2} \leqslant \lambda_{l} l^{-p} \leqslant C_{3}<\infty
$$

при больших l, и пусть оператор $\mathfrak{A}_{1}$ q-подчинен оператору $\mathfrak{A}_{0}, q<1$. Тогда если $p(1-q) \geqslant 1$, то система корневых векторов оператора $\mathfrak{A}=\mathfrak{A}_{0}+\mathfrak{A}_{1}$ образует безусловный базис со скобками. Если же $p(1-q)<1$, то она образует базис для метода суммирования Абеля-Лидского любого порядка $r>r_{0}=p^{-1}-(1-q)$.

Этот результат принадлежит Кацнельсону [57], [58] (при $q \geqslant 0$ ). Другое доказательство теоремы для случая $p(1-q)=1$ см. в [78] или [76; гл. I, $\S 6]$. Для $p(1-q)>1$ наша формулировка здесь на самом деле загрубленная, результат допускает сушественные уточнения, в особенности если $q<0$ и/или в $(2.41)$ известна усиленная оценка остатка, как это обычно бьвает для эллиптических операторов. См. [4; п. 6.2] и приведенную там литературу, в частности, работу [75] о квадратичной близости базиса со скобками к ортонормированному базису при $p(1-q)>1$ и работу [2].

Пусть теперь операторы $\mathfrak{A}_{0}$ и $\mathfrak{A}$ заданы полуторалинейными формами $\Psi_{0}(\mathfrak{u}, \mathfrak{v})$ и $\Psi(\mathfrak{u}, \mathfrak{v})$, причем первая форма является действительной частью второй формы, т.е. полусуммой форм $\Psi(u, v)$ и $\overline{\Psi(v, u)}$. Пусть это замкнутые формы с общей плотной в $\mathfrak{H}$ областью определения $\mathfrak{H}_{1}$, представляющей собой гильбертово пространство с нормой $\|\cdot\|_{1}$, непрерьвно и компактно вложенное в $\mathfrak{H}$. Предположим, что

$$
\varepsilon\|\mathfrak{u}\|_{1}^{2} \leqslant \Psi_{0}(\mathfrak{u}, \mathfrak{u}) \text { и }\left|\Psi_{0}(\mathfrak{u}, \mathfrak{v})\right|+|\Psi(\mathfrak{u}, \mathfrak{v})| \leqslant C_{4}\|\mathfrak{u}\|_{1}\|\mathfrak{v}\|_{1}
$$

при некоторых $\varepsilon>0$ и $C_{4}>0$. Согласно “теоремам о представлении” из [56] эти две формы определяют самосопряженный оператор $\mathfrak{A}_{0}$ и “ $m$-секториальньй” оператор $\mathfrak{A}$, оба с компактной резольвентой. Для разности $\psi(\mathfrak{u}, \mathfrak{v})$ этих форм используем определение $q$-подчиненности форме $\Psi_{0}(\mathfrak{u}, \mathfrak{v})$ из п. 2.8. Следующее предложение взято из [7], но тоже приводится здесь в загрубленной форме. 
ПРЕДЛОЖЕНИЕ 3.6. Пусть разность форм $\Psi$ u $\Psi_{0}$ q-подчинена форме $\Psi_{0}$, $0 \leqslant q<1$. Тогда справедливы такие же заключения о системе корневых функций оператора $\mathfrak{A}$, как в предьдущем предложении.

5. Пусть снова $\mathfrak{T}$ - компактный оператор. Его $s$-иислами $s_{j}(\mathfrak{T})$ назьваются ненулевые собственные значения неотрицательного оператора $\left(\mathfrak{T}^{*} \mathfrak{T}\right)^{1 / 2}$, занумерованные в порядке невозрастания с учетом кратности. Если $\mathfrak{B}-$ ограниченный оператор, то $s_{j}(\mathfrak{B T})$ и $s_{j}(\mathfrak{T} \mathfrak{B})$ не превосходят $\|\mathfrak{B}\| s_{j}(\mathfrak{T})$. При этом $\mathfrak{B}$ может быть и оператором из одного гильбертова пространства в другое. Если $\mathfrak{T}$ - самосопряженньй оператор, то $s_{j}(\mathfrak{T})$ - модули его ненулевых собственных значений и собственные значения оператоpa $|\mathfrak{T}|=\left(\mathfrak{T}^{2}\right)^{1 / 2}$ с теми же собственными векторами, что и у $\mathfrak{T}$. См. [41; гл. II]

6. Если $\mathfrak{H}=H^{t_{1}}(M)$, где $M$ - замкнутое $n$-мерное многообразие, и $T$ - оператор, действующий ограниченным образом из $H^{t_{1}}(M)$ в $H^{t_{2}}(M), t=t_{2}-t_{1}>0$, то $s_{l}(T) \leqslant C l^{-t / n}$ и аналогичная оценка верна для модулей собственных значений. Ср. [41; гл. II, §3], а также [105]. Для гладких многообразий это объяснено также в [4; пп. 5.4 и 6.1$]$, а на липшицевы $n$-мерные поверхности переносится при помощи липшицевых диффеоморфизмов, ср. ниже доказательство оценок (5.3). Такие же оценки верны в случае, когда $M$ - область в $\mathbb{R}^{n}$ с липшицевой границей.

7. Комплексно сопряженным к $\mathfrak{T}$ оператором называется оператор $\overline{\mathfrak{T}}$, действующий по формуле $\overline{\mathfrak{T}} \mathfrak{u}=\overline{\mathfrak{T}} \overline{\mathfrak{u}}$. Очевидно, что $\mathfrak{T}^{*}=\overline{\mathfrak{T}^{\prime}}$, где $\mathfrak{T}^{\prime}-$ транспонированньй к $\mathfrak{T}$ оператор. Если $\mathfrak{T}=\mathfrak{T}^{\prime}$, т.е. оператор комплексно симметричен, то $\mathfrak{T}^{*}=\overline{\mathfrak{T}}$. В этом случае полную минимальную систему корневых векторов (если такая система существует) можно подчинить условию “вещественной ортогональности” $\left(\mathfrak{u}_{j}, \overline{\mathfrak{u}}_{k}\right)=\delta_{j k}$. См. $[1 ; \S 31]$ или $[14 ;$ п. 5.2].

3.3. Задачи, близкие к самосопряженным. Мы теперь отказываемся от предположений о формальной самосопряженности. Но главная часть оператора $L$ по-прежнему предполагается эрмитовой. Сначала рассмотрим граничное условие $B u=0$.

1. Случай гладкой границы. Оператор $\mathscr{L}$ по-прежнему определяется как оператор в $H^{0}(\Omega)$ с областью определения $H^{2}(\Omega, B)$. С двигая спектральный параметр, мы можем предположить, что этот оператор обратим. Тогда его степени $\mathscr{L}^{\theta}, 0<\theta=$ $t / 2<1$, по-прежнему изоморфно отображают $\left[H^{0}(\Omega), H^{2}(\Omega, B)\right]_{\theta}$ на $H^{0}(\Omega)[73]$ и это пространство совпадает с $H^{t}(\Omega)$ при $t<3 / 2$ и с $H^{t}(\Omega, B)$ при $3 / 2<t \leqslant 2[43]$, [116].

Проше всего случай, когда $b_{j}=0$ и $b$-эрмитова матрица, а матрица $c$ не является эрмитовой. Тогда операторы $\mathscr{L}_{\text {и }} \mathscr{L}^{(*)}$ имеют общую область определения $H^{2}(\Omega, B)$. Так как $p=2 / n$ и $q=0$, то $p(1-q)=2 / n$. Система корневых функций оператора $\mathscr{L}$ является безусловным базисом со скобками при $n=2$, а при бо́льших $n-$ базисом для метода суммирования Абеля-Лидского любого порядка $r>(n-2) / 2$ в $H^{t}(\Omega)$, $0<t<3 / 2$, и в $H(\Omega, B), 3 / 2<t \leqslant 2$, в силу замечания 3.4 и предложения 3.5 .

В общем случае $\mathscr{L}$ и $\mathscr{L}^{(*)}$ имеют разные области определения и мы должны сравнивать форму $\Phi(u, v)$, отвечающую оператору $\mathscr{L}$, с ее вешественной частью $\operatorname{Re} \Phi(u, v)$. Как легко проверить, преобразуя последнюю интегрированием по частям, ей отвечает граничная задача той же структуры, а разность форм при $v=u$ оценивается через $\|u\|_{1, \Omega}\|u\|_{0, \Omega}$. Поэтому разность форм $1 / 2$-подчинена форме $\operatorname{Re} \Phi$ (см. п. 2.8). Это приводит к базисности для метода суммирования Абеля-Лидского системы корневых 
функций любого порядка $r>(n-1) / 2$ в $H^{t}(\Omega), 0<t<3 / 2$, и в $H^{t}(\Omega, B), 3 / 2<t \leqslant 2$, в силу предложения 3.6.

Сохраняется также асимптотика (1.25) собственных значений.

При ослаблении предположений о гладкости (см. п. 1.5) сохраняются те же утверждения о базисности со скобками или базисности Абеля-Лидского, а оценка остатка в асимптотике собственных значений не хуже, чем в случае самосопряженного оператора.

2. Случай липшицевой границы. В этом случае снова можно рассматривать два оператора $\mathscr{L}$, как в $\S 2$. Первый определяется как оператор из $H^{1}(\Omega)$ в $H_{0}^{-1}(\Omega)$, и для него остается в силе теорема 2.1. Второй - это оператор в $H^{0}(\Omega)$ с областью определения, содержашейся в $H^{1}(\Omega)$. Очень существенно, что область определения квадратного корня $\mathscr{M}$ из него совпадает с $H^{1}(\Omega)$. Это следует из того, что, как мы знаем, форма $\Phi(u, v)$ 1/2-подчинена ее вещественной части, см. теорему А в [82].

Поэтому сохраняются такие же утверждения о базисности корневых функций со скобками и базисности Абеля-Лидского в пространствах $H^{t}(\Omega), t<3 / 2$, как в случае гладкой границы.

3. Осталось добавить несколько слов о спектральной задаче Дирихле при отсутствии формальной самосопряженности. Она остается слабым возмущением самосопряженной задачи Дирихле. Поэтому в основном сохраняются утверждения о базисности корневых функций со скобками или их базисности для метода суммирования Абеля-Лидского, приведенные вьше в этом пункте для задач с граничным условием первого порядка. Разница только в том, что граница между пространствами $H^{t}(\Omega)$, в которых "не удерживается" условие Дирихле, и пространствами $H^{t}(\Omega, D)$ находится в точке $t=1 / 2$, а не $3 / 2$.

3.4. Системы с другими особенностями в младшем коэффициенте. Мы приведем сначала элементарные соображения, позволяюшие рассмотреть вместо $1 /|x|$ особенности типа $1 /|x|^{\beta}$ с $\beta<2$. Сейчас мы считаем, что $n \geqslant 3$, так как будем пользоваться неравенством Харди (1.34).

ПРЕДЛОЖЕНИЕ 3.7. Форма $\left(|x|^{-\beta} u(x), v(x)\right)_{\Omega}$ является $\beta / 2$-подчиненной форме $\Phi(u, v)_{\Omega}$ при $0<\beta<2$.

ДокАЗАТЕЛЬство. Оператор умножения на $1 /|x|$ можно рассматривать как самосопряженньй неотрицательный оператор в $H^{0}(\Omega)$ с областью определения $H^{1}(\Omega)$. Интерполируя и используя его степени, получаем, что при $\alpha \in(0,1)$ оператор умножения на $1 /|x|^{\alpha}$ действует ограниченным образом из $H^{\alpha}(\Omega)$ в $H^{0}(\Omega)$ :

$$
\int_{\Omega}|x|^{-2 \alpha}|u(x)|^{2} d x \leqslant\|u\|_{\alpha, \Omega}^{2}
$$

Кроме того, при $\alpha \in(0,1)$

$$
\|u\|_{\alpha, \Omega}^{2} \leqslant C_{\alpha}\left(\|u\|_{1, \Omega}\right)^{2 \alpha}\left(\|u\|_{0, \Omega}\right)^{2-2 \alpha} .
$$

Это так назьваемое неравенство Гальярдо-Ниренберга (ср. [17; п. 6.8]). Оно получается заменой функции $u$ ее продолжением $E u$ на $\mathbb{R}^{n}$, где оператор продолжения ограничен в соболевских нормах нулевого и первого порядка, т.е. $\|E u\|_{t, \mathbb{R}^{n}} \leqslant C\|u\|_{t, \Omega}$ 
при $t=0,1$ (см. п. 2.1), и применением неравенства Гёльдера к выражению $\|E u\|_{\alpha, \mathbb{R}^{n}}^{2}$, записанному через преобразование Фурье функции $E u$. Остается положить $\alpha=\beta / 2$.

Если форма $\left(c_{2} u, v\right)_{\Omega} \quad q$-подчинена форме $\Phi(u, v)$ при некотором $q \in(1 / 2,1)$, то рассмотрения в пा. 1.6 и 2.9 , конечно, нуждаются в поправках. Но мы не будем тратить здесь место на их обсуждение.

Из многочисленных работ, посвященных исследованию граничных задач для уравнения Шрёдингера с особенностями в потенщиале, упомянем работы [22] (задача Дирихле, неспектральные вопросы), [49] (потенциалы с неглубокими или влияющими на область определения оператора глубокими особенностями, спектральные асимптотики), [81] (замкнутое многообразие, особенности потенциала могут быть очень глубокими), [54] ( $n \leqslant 3$, некоторая степень потенциала суммируема), [117] (потенциал из так назьваемого класса Като, сходимость рядов Фурье по собственным функциям на компактах внутри области), [102] (потенциалы из класса мультипликаторов из $H^{1}$ в $\left.H^{-1}\right)$ и [112] ( $\left.n=1\right)$. В печати находятся интересные работы Савчука и Шкаликова (Труды MМO) и Мазьи и Вербицкого (Acta Math.). Упомянем еще обзор [55] работ по условиям существенной самосопряженности оператора Шрёдингера с сингулярными потенциалами в $\mathbb{R}^{n}$. См. также ссылки во всех этих работах.

\section{Глава II. Задачи со спектральным параметром в граничном условии или условии сопряжения}

\section{$\S 4$. Задачи со спектральным параметром на гладкой границе}

4.1. Постановка спектральных задач. Систему в этой главе мы будем записьвать в виде

$$
\sum_{j, k} \partial_{j}\left[a_{j k}(x) \partial_{k} u(x)\right]-\sum_{j} b_{j}(x) \partial_{j} u(x)-c(x) u(x)+\omega^{2} u(x)=0
$$

и считать, что она задана в $\mathbb{R}^{n}$. В этом и следующем параграфах будем назьвать систему (4.1) формально самосопряженной, если $b_{j k}=0, c=c^{*}$ и $\omega^{2}$ вешественно. Поверхность $\Gamma$ разбивает свое дополнение на ограниченную область $\Omega=\Omega^{+}$и неограниченную область $\Omega^{-}$. Для наглядности и простоты мы считаем поверхность $\Gamma$ связной, хотя можно было бы рассматривать и более общую ситуащию (ср. [86]).

Граница и коэффициенты предполагаются в этом параграфе бесконечно гладкими, коэффициенты $a_{j k}(x)$ подчинены условиям сильной эллиптичности, симметрии (1.3) и II и постоянны вне шара $O_{R}$ достаточно большого радиуса $R$, коэффициенты $b_{j}(x)$ и $c(x)$ равны нулю вне этого шара. (Но в п. 4.3 мы рассмотрим менее общие системы.) Имитируя обычные предположения об уравнении Гельмгольца и системе Ламе, мы предположим, что параметр $\omega=\omega^{\prime}+i \omega^{\prime \prime}$ фиксирован в верхней полуплоскости, при этом либо $\omega^{\prime \prime}>0$, либо $\omega \geqslant 0$. Все такие $\omega$ назовем допустимылми. (В физических задачах обычно $\omega=0$ - случай равновесия, $\omega>0$ - случай установившихся колебаний и $\omega^{\prime}>0, \omega^{\prime \prime}>0$ - случай затухаюших колебаний.) Кроме того, для упрошения 
изложения мы примем, что $\omega \neq 0$ при $n=2$ в задачах II-IV.$^{4}$ Постоянство коэффициентов при больших $|x|$ позволит ниже наложить на решения естественные условия на бесконечности.

Мы рассмотрим четыре спектральных задачи.

ЗАДАчА І. Ищется решение системы (4.1) в $\Omega^{+}$с граничным условием

$$
\partial_{\nu_{a}} u^{+}+\mu u^{+}=0 \text { на } \Gamma \text {. }
$$

ЗАДАчА II. Ишется решение системы (4.1) в $\Omega^{-}$с граничным условием

$$
\partial_{\nu_{a}} u^{-}=\mu u^{-} \text {на } \Gamma \text {. }
$$

ЗАДАчА III. Ишется решение системы (4.1) вне Г с условиями сопряжения

$$
u^{+}=u^{-} \text {и } \partial_{\nu_{a}} u^{-}-\partial_{\nu_{a}} u^{+}=\mu u^{ \pm} \text {на } Г .
$$

ЗАДАчА IV. Ищется решение системы (4.1) вне Г с условиями сопряжения

$$
\partial_{\nu_{a}} u^{+}=\partial_{\nu_{a}} u^{-} \text {и } \partial_{\nu_{a}} u^{ \pm}=\mu\left[u^{-}-u^{+}\right] \text {на } \Gamma \text {. }
$$

Во всех задачах $\mu$ - спектральньй параметр и $\partial_{\nu_{a}}-$ конормальная производная, см. (1.5). Рассматривая задачу I, можно предполагать систему заданной только в $\bar{\Omega}$ и не выделять слагаемое $\omega^{2} u$ из младшего члена, но нам будет удобно сохранить это слагаемое для единообразия обозначений. Рассматривая задачу II, можно считать систему заданной только вне $\Omega$.

В задачах II-IV мыналожим на решения условия убьвания на бесконечности, связав их с выбором фундаментального решения для системы (4.1). Итак, пусть $|x|>R$.

При невещественном $\omega$ матрица $\omega^{2} I-a(\xi)$, где $a(\xi)$ - главньй символ $\sum a_{j k} \xi_{j} \xi_{k}$, всюду невырождена и мы определяем фундаментальное решение $E_{\omega}(x)$ формулой

$$
E_{\omega}(x)=F^{-1}\left[\omega^{2} I-a(\xi)\right]^{-1},
$$

где $F^{-1}$ - обратное преобразование Фурье в смысле обобщенных функций. Это интеграл по $\xi$, который на бесконечности регуляризуется следующим образом: под знаком интеграла помещается множитель $\left(1+|\xi|^{2}\right)^{-s}$, а перед интегралом ставится оператор $(1-\Delta)^{s}$, где $s-$ любое натуральное число, для которого $2 s+2>n$. Матрица $E_{\omega}(x)$ эрмитова при $\omega^{\prime}=0$, четна по $x$, аналитична при $x \neq 0$, имеет при $x=0$ особенность порядка $O\left(|x|^{2-n}\right)$ при $n \geqslant 3$ и логарифмическую особенность при $n=2$ и убьвает на бесконечности в силу бесконечной гладкости ее преобразования Фурье. Более того, убывание является экспоненциальным, в чем можно убедиться, заменяя поверхность интегрирования $\mathbb{R}^{n}$ подходящей поверхностью в $\mathbb{C}^{n}$, зависящей от направления $x /|x|$

\footnotetext{
${ }^{4}$ Мыне хотим тратить здесь место на случай, когда фундаментальное решение имеет логариффмическое поведение на бесконечности. Конечно, этот случай тоже можно рассмотреть. В частности, общеизвестны результаты по внешним задачам для уравнения Лапласа на плоскости. См., например, [85].
} 
(см., например, [45]). Соответственно на решения на бесконечности накладываются условия

$$
u(x)=O(\exp (-\delta|x|)) \quad \text { и } \partial_{k} u(x)=O(\exp (-\delta|x|))
$$

с достаточно малым положительным $\delta$.

При $\omega=0, n \geqslant 3$ фундаментальное решение $E_{\omega}(x)$ определяется так же и является аналитической при $x \neq 0$ матрицей, эрмитовой, четной по $x$ и положительно однородной степени $2-n$. (Cp. [94].) Соответственно на решения $u(x)$ накладываются условия

$$
u(x)=O\left(|x|^{2-n}\right) \quad \text { и } \partial_{k} u(x)=O\left(|x|^{1-n}\right) .
$$

Существенно сложнее обстоит дело при $\omega>0$. При этих $\omega$ нам нужно выделить фундаментальное решение, аналогичное фундаментальному решению для уравнения Гельмгольца $\Delta u+\omega^{2} u=0$ при $n=3$ вида $-(4 \pi|x|)^{-1} \exp (i \omega|x|)$. Для этого мы должны наложить дополнительное условие на главньй символ $a(\xi)$ при больших $|x|$ (cp. [121], [122] и [97], [98]). Так как это эрмитова матрица, то она подобна вешественной диагональной матрице. Обозначим ее собственные значения через $d_{p}(\xi)$. Они положительны и однородны степени 2.

УСлОвИЕ V (для $\omega>0)$. Собственные значения $d_{p}(\xi)$ при $\xi \neq 0$ имеют кратности, не зависящие от $\xi$. Поверхности $S_{p}$ в $\mathbb{R}^{n}$, определяемые уравнениями $d_{p}(\xi)=\omega^{2}$, строго выпукль. Более точно, они звездны относительно начала координат, в каждой их точке все главные кривизны имеют один и тот же знак, а радиус-вектор каждой такой точки, проведенный в нее из начала координат, образует в ней острый угол с направлением внешней нормали к $S_{p}$ в этой точке.

Это условие выполнено, например, в случае уравнений Гельмгольца и Ламе (см. п. 1.2). При этом условии фундаментальное решение удобно определить формулой

$$
E_{\omega}(x)=\lim _{\varepsilon \downarrow 0} E_{\omega+i \varepsilon}(x)
$$

(вторая возможность: $\varepsilon \uparrow 0)$. Оно остается четным по $x$ и аналитическим при $x \neq 0$.

Следуя [121], [122] и [97], [98], удается детально исследовать структуру этого фундаментального решения и выяснить его асимптотическое поведение на бесконечности. Детали читатель найдет ниже в предложении 4.12 и в $[9]$. Пусть $d_{1}(\xi), \ldots, d_{q}(\xi)-$ все попарно разные собственные значения главного символа $a(\xi)$.

Тогда фундаментальное решение (4.9) можно представить в виде суммы из $q$ слагаемых $E_{\omega, p}(x), p=1, \ldots, q$, каждое из которых имеет полную асимптотику при $x \rightarrow \infty$ по убываюшим отрицательным степеням $|x|$ с осциллируюшими коэффициентами. Начало этой асимптотики имеет вид

$$
E_{\omega, p}(x)=|x|^{-(n-1) / 2} e^{i x \cdot \xi^{p}} C_{p}(\alpha)+O\left(|x|^{-(n+1) / 2}\right) .
$$

Здесь $\alpha=x /|x|, \xi^{p}=\xi^{p}(\alpha)$ - радиус-вектор точки на $S_{p}$, в которой внешняя нормаль имеет направление $\alpha$, и $C_{p}(\alpha)$ - некоторая матрица, гладкая по $\alpha$. Соответственно мы 
должны предположить, что любое решение $u(x)$ системы (4.1) состоит из слагаемых $u_{1}(x), \ldots, u_{q}(x)$ с асимптотикой, начало которой имеет вид

$$
u_{p}(x)=|x|^{-(n-1) / 2} e^{i x \cdot \xi^{p}} c_{p}(\alpha)+O\left(|x|^{-(n+1) / 2}\right),
$$

где $c_{p}(\alpha)$ - некоторьй вектор, гладко зависяший от $\alpha$. С учетом этой асимптотики мы налагаем следуюшие условия излучения на решения системы (4.1):

$$
u(x)=u_{1}(x)+\cdots+u_{q}(x),
$$

где

$$
u_{p}(x)=O\left(|x|^{-(n-1) / 2}\right) \quad \text { и } \partial_{k} u_{p}(x)-i \xi_{k}^{p}(\alpha) u_{p}(x)=O\left(|x|^{-(n+1) / 2}\right) .
$$

Этим условиям удовлетворяет, в частности, фундаментальное решение (4.9).

Отметим еше, что при вешественных старших коэффициентах $a_{j k}$ матрица $E_{\omega}(x)$ симметрична при любом допустимом $\omega$, т.е. совпадает с транспонированной матрицей.

Теперь задачи I-IV сформулированы полностью. Для уравнения Гельмгольца они были поставлены в книге [126] и предшествовавших ей работах ее авторов. В [1], [42] эти задачи были проанализированы в случае гладкой границы Г и в [15] в случае липшицевой границы. См. также переработанное английское издание [14] книги [126] с добавлением [1]. Аналогичные задачи для системы Ламе рассмотрены в [12] и для обших систем (4.1) в [9], но там коэффициенты предполагались постоянными и старшие коэффициенты вешественными. Случай постоянных коэффициентов мы рассмотрим в п. 4.3 при помощи потенщиалов, и в этом случае получается сушественная дополнительная информация, а в ближайшем п. 4.2 мы будем пользоваться только обшими средствами теории эллиптических операторов и задач.

4.2. Сведе́ние задач I-IV к эллиптическим уравнениям на Г и спектральные свойства соответствующих операторов. Через $D_{\omega}^{ \pm}$будем обозначать внутреннюю и внешнюю задачи Дирихле для системы (4.1) с граничным условием $u^{ \pm}=h$. Через $N_{\omega}^{ \pm}$обозначим внутреннюю и внешнюю задачи Неймана для этой системы с граничньм условием $\partial_{\nu_{a}} u^{ \pm}=g$. Во внешних задачах решение подчиняется нашим условиям на бесконечности. В п. 1.1 мы отметили эллиптичность внутренних задач Дирихле и Неймана. Разумеется, она имеет место и для внешних задач. Все условия (4.3)-(4.5) также эллиптичны. Общие эллиптические задачи сопряжения рассматривались в [113].

Из эллиптичности следует фредгольмовость внутренних задач $D_{\omega}^{+}$и $N_{\omega}^{+}$в соболевских пространствах, при этом

$$
h \in H^{t}(\Gamma) \Leftrightarrow u \in H^{t+1 / 2}(\Omega) \quad \text { и } g \in H^{t}(\Gamma) \Leftrightarrow u \in H^{t+3 / 2}(\Omega)
$$

соответственно для задач $D_{\omega}^{+}$и $N_{\omega}^{+}$. Для простоты можно считать, что здесь $t>0$.

Решения задачи $D_{\omega}^{-}$с $h \in H^{t}(\Gamma)$ можно искать в пространстве $H_{\mathrm{loc}}^{t+1 / 2}\left(\Omega^{-}\right)$. Аналогично решение задачи $N_{\omega}^{-}$с $g \in H^{t}(\Gamma)$ можно искать в $H_{\mathrm{loc}}^{t+3 / 2}\left(\Omega^{-}\right)$. Но при невещественном $\omega$ ввиду быстрого убывания решений они оказьваются в обычных соболевских пространствах, так что индекс loc можно опустить. 
Задача I. Внутренние задачи Дирихле и Неймана для системы (4.1) имеют нулевой индекс: это проверяется при помощи гомотопий. Спектры соответствующих операторов в $\Omega$, отвечаюших задачам с однородными граничными условиями, дискретны и полуограничены снизу. Для простоты мы сразу предположим, что при данном $\omega$ для этих задач имеет место единственность, или, что то же, однозначная разрешимость. Тогда сушествует обратимьй оператор $\mathscr{T}_{\omega}^{+}$, переводяший данные Дирихле в данные Неймана с обратным знаком:

$$
\mathscr{T}_{\omega}^{+} u^{+}=-\partial_{\nu_{a}} u^{+}
$$

Действительно, по $u^{+}$определяется $u(x)$ в $\Omega$ как решение задачи Дирихле, это кограничньй оператор, затем к $u$ применяется граничньй оператор взятия конормальной производной $\partial_{\nu_{a}} u^{+}(x)$ с изменением знака. Из общих фактов теории эллиптических задач (ср., например, [36], [69; гл. 6, § 5], [107; гл. 3]) следует, что $\mathscr{T}_{\omega}^{+}$- эллиптический ПДО порядка 1. Главньй символ ПДО $\mathscr{T}_{\omega}^{+}$допускает явное вычисление. При этом вычислении в точке на границе следует вьпрямить границу, отбросить младшие члены и заморозить значения старших коэффициентов. Результат будет такой, как в случае постоянных коэфрициентов, см. ниже п. 4.3.

Мы видим, что $\mathscr{T}_{\omega}^{+}-$ограниченный и обратимьй оператор из $H^{t}(\Gamma)$ в $H^{t-1}(\Gamma)$ при всех $t$. В $H^{0}(\Gamma)$ это самосопряженньй оператор (с областью определения $H^{1}(\Gamma)$ ), если система формально самосопряженная. Действительно, в этом случае при помощи формулы Грина, т.е. интегрированием по частям, получаем

$$
\left(\partial_{\nu_{a}} u^{+}, v^{+}\right)_{\Gamma}=\left(u^{+}, \partial_{\nu_{a}} v^{+}\right)_{\Gamma}
$$

Если, кроме того, $c=0$ и $\omega$ чисто мнимое, то оператор $\mathscr{T}_{\omega}^{+}$отрищателен:

$$
-\int_{\Omega} E(u, u) d x-|\omega|^{2} \int_{\Omega}|u|^{2} d x=\left(\mathscr{T}_{\omega}^{+} u, u\right)_{\Gamma}
$$

Следовательно, главньй символ ПДО $\mathscr{T}_{\omega}^{+}$эрмитов и отрицательно определен. Если этот оператор не является самосопряженным, то он отличается от самосопряженного оператора $\operatorname{Re} \mathscr{T}_{\omega}^{+}$на слагаемое (ПДО) порядка не вьше 0 и остается полуограниченным сверху оператором.

Задача I эквивалентна уравнению

$$
\mathscr{T}_{\omega}^{+} \varphi=\mu \varphi
$$

где $\varphi=u^{+}$, в том смысле, что граничные значения собственных функций и собственные значения задачи являются собственными функциями и собственными значениями оператора $\mathscr{T}_{\omega}^{+}$. Эта эквивалентность является полной в самосопряженном случае. Если самосопряженности нет, то, вообще говоря, у оператора $\mathscr{T}_{\omega}^{+}$могут быть и присоединенные функции. Мы не будем искать соответствуюшие решения задачи I и далее интересуемся только спектральными свойствами оператора $\mathfrak{T}_{\omega}^{+}$. Аналогичной точки зрения мы придерживаемся ниже в отношении остальных задач II-IV. 
Для собственных значений оператора $\mathscr{T}_{\omega}^{+}$имеет место формула

$$
\mu_{j}=-C_{n} j^{1 /(n-1)}+O(1) .
$$

Для самосопряженного оператора это следует из теоремы Хёрмандера 1968 г., см. [46; т. 4] $(m=1)$, и Иврия [47] $(m>1)$, на несамосопряженные операторы результат распространяется при помоши теоремы Маркуса-Мацаева, упомянутой в п. 2.8 (предложение 2.16) и п. 3.2. Положительная постоянная $C_{n}$ вычисляется через функцию распределения собственных значений главного символа.

В обозначениях п. 3.2 сейчас $p=1 /(n-1)$ и $q \leqslant 0$. Поэтому при отсутствии самосопряженности мнимые части собственных значений, по крайней мере, ограничены (см. (3.6)), при $n=2$ из корневых функций строится безусловньй базис со скобками во всех $H^{t}(\Gamma)$, а при $n>2$ ряды по корневым функциям, по крайней мере, суммируются во всех $H^{t}(\Gamma)$ методом Абеля-Лидского любого порядка $r>n-2$ (см. замечание 3.4 и предложение 3.5$)$.

В пространствах $H^{t}(\Gamma)$ ситуация аналогичная. В этом можно убедиться, используя степени оператора $-\mathscr{T}_{\omega}^{+}$с чисто мнимым $\omega$.

Подведем итоги:

ТЕОРема 4.1. При предположениях об однозначной разрешимости внутренних задач Дирихле и Неймана оператор $\mathscr{T}_{\omega}^{+}$является әллиптическим обратимымм ПДО первого порядка на Г с эрмитовым отрицательно определенным главным символом. В случае формальной самосопряженности системь (4.1) это самосопряженныи оператор в $H^{0}(\Omega)$, его собственные функиии принадлежат $C^{\infty}(\Gamma)$ и составленный из них ортонормированный базис в $H^{0}(\Gamma)$ является безусловным базисом во всех соболевских пространствах $H^{t}(\Gamma)$. Собственные значения имеют асимптотику (4.19). В общем случае оператор $\mathscr{T}_{\omega}^{+}$отличается от своей действительной части на оператор порядка не выше 0; корневье функции принадлежат $C^{\infty}$ и при $n=2$ образуют безусловный базис со скобками, а при $n>2$ - базис для метода суммирования Абеля-Лидского любого порядка больше $n-2$ во всех $H^{t}(\Gamma)$. Собственные значения имеют ту же асимптотику, их мнимые части ограничень.

ЗАмЕЧАНИЕ 4.2. При $n=2$ результаты можно существенно усилить, если собственные значения главного символа имеют постоянные кратности. А именно, для $\mu_{l}$ строятся тогда асимптотические ряды по убывающим степеням номера $l$. При отсутствии самосопряженности скобки, которые надо расставлять в ряде Фурье по корневым функциям, содержат ограниченное число слагаемых. Для элементов базиса в соответствуюших подпространствах строятся асимптотические разложения по степеням номера подпространства. См. по этому поводу [3] и указанные там ссылки. Аналогичное замечание относится к операторам, отвечающим дальнейшим задачам II-IV.

ЗАмЕЧАНИЕ 4.3. В частности, в отношении второй формуль для $R$-матриць (см. формулу (11) во введении) мы видим, что при условиях самосопряженности она справедлива в смысле сходимости в $H^{t}(\Gamma)$, если $g \in H^{t-1}(\Gamma)$. При достаточно большом $t$ имеет место равномерная сходимость. Если $n=3$, то для этого заведомо достаточно, чтобы функция $g$ была класса $C^{1}(\Gamma)$. Все это получается для любой системы, не только для уравнения Шрёдингера. 
Другие варианты "метода $R$-матрищы” можно связать с задачами II-IV. Мы не будем на этом останавливаться.

2. Задача II. Рассматривая эту задачу, примем, что внешние задачи Дирихле и Неймана однозначно разрешимы. Тогда аналогично (4.15) вводится оператор

$$
\mathscr{T}_{\omega}^{-} u^{-}=\partial_{\nu_{a}} u^{-} .
$$

Следующее замечание облегчает анализ этого оператора средствами теории эллиптических задач.

ЗАмечАние 4.4. Пусть область $\Omega$ лежит в $O_{R}$ и коэффициенты постоянны вне $O_{R}$. Фиксируя $R^{\prime}>R$, мы можем рассматривать решение $u$ системы (4.1) в $\Omega_{R^{\prime}}$ как имеющее заданное граничное значение $u^{+}$или $\partial_{\nu_{a}} u^{+}$на границе шара $O_{R^{\prime}}$.

Более того, можно эквивалентным образом заменить систему (4.1) вне $O_{R^{\prime}}$ (с условиями на бесконечности из п. 4.1) эллиптическим псевдодифференциальным условием 1-го порядка на границе шара $O_{R^{\prime}}$, по крайней мере при вещественных $a_{j k}=a_{k j}^{\prime}$, и тем самьм свести задачу II к эллиптической задаче в $\Omega_{R^{\prime}}^{-}$.

Для этого надо записать представление решений вне $O_{R^{\prime}}$ через их данные Коши на поверхности этого шара (см. ниже вторую формулу в (4.33)) и перейти в этом представлении на границу шара. В полученном соотношении заменить внешние данные Коши на внутренние данные Коши. Получится нужное граничное условие на поверхности шара $O_{R^{\prime}}$.

Аналогичное замечание можно сделать к рассматриваемьм ниже задачам III и IV.

Для уравнения Гельмгольца это отмечалось в [1], см. также [14] и [9].

Теперь видно, что $\mathscr{T}_{\omega}^{-}-$эллиптический ПДО порядка 1 . Если система формально самосопряженная и $\omega^{\prime}=0$, то это самосопряженньй оператор; при этом если $b_{j k}$ и $c-$ нули, то он отрицательно определен. Это проверяется при помощи формулы Грина в $\Omega_{R}^{-}$с последуюшим предельньм переходом при $R \rightarrow \infty$. Следовательно, главный символ эрмитов и отрищательно определен. Если $\mathscr{T}_{\omega}^{-}$не является самосопряженным оператором, то это возмущение самосопряженного оператора $\operatorname{Re} \mathscr{T}_{\omega}^{-}$слагаемым порядка не вьше 0 и полуограниченный сверху оператор.

Задача II эквивалентна уравнению

$$
\mathscr{T}_{\omega}^{-} \varphi=\mu \varphi,
$$

где $\varphi=u^{-}$, в смысле совпадения собственных функций (на Г) и собственных значений. Мыне будем формулировать теорему о спектральных свойствах оператора $\mathscr{T}_{\omega}^{-}$, почти дословно повторяюшую теорему 4.1.

Операторы, обратные к $\mathscr{T}_{\omega}^{ \pm}$, д дальнейшем будут обозначаться через $T_{\omega}^{ \pm}$. Это эллиптические ПДО порядка -1 .

3. Задача III. Предположим, что внутренние и внешние задачи Дирихле и Неймана однозначно разрешимы при данном $\omega$ и что решение системы (4.1) в $\mathbb{R}^{n}$, удовлетворяющее условиям на бесконечности из $n .4 .1$, тождественно равно нулю.

Рассмотрим оператор $\mathscr{T}_{\omega}^{+}+\mathscr{T}_{\omega}^{-}$. Если $u^{ \pm}-$совпадающие данные Дирихле решений в $\Omega^{ \pm}$, то он переводит их в $\partial_{\nu_{a}} u^{-}-\partial_{\nu_{a}} u^{+}$. Это эллиптический ПДО 1-го порядка 
с эрмитовьм отрицательно определенным главным символом (так как $\mathscr{T}_{\omega}^{ \pm}$имеют эрмитовы отрицательно определенные символы), следовательно, фредгольмов оператор из $H^{t}(\Gamma)$ в $H^{t-1}(\Gamma)$ с нулевым индексом. Его обратимость проверяется следующим образом: если он переводит $u^{ \pm}$в 0 , то получается решение в $\Omega^{ \pm}$без скачков в данных Коши на $Г$. В такой ситуации особенность вдоль $\Gamma$, как известно, стирается и получается решение системы в $\mathbb{R}^{n}$. По предположению, оно равно нулю, так что наш оператор ничего не аннулирует, значит, обратим. Обратный оператор в дальнейшем будет обозначаться через $A_{\omega}$.

Задача III эквивалентна уравнению

$$
\left(\mathscr{T}_{\omega}^{+}+\mathscr{T}_{\omega}^{-}\right) \varphi=\mu \varphi
$$

где $\varphi=u^{ \pm}$, в смысле совпадения собственных функций и собственных значений.

Спектральные свойства суммы $\mathscr{T}_{\omega}^{+}+\mathscr{T}_{\omega}^{-}$совершенно аналогичны спектральным свойствам оператора $\mathscr{T}_{\omega}^{-}$. Самосопряженность этой суммы имеет место в случае формально самосопряженной системы с $\omega^{\prime}=0$.

4. Задача IV. Сделаем такие же предположения, как при рассмотрении предыдущей задачи. Сопоставим задаче IV оператор $T_{\omega}$, определив его на решениях системы (4.1) в $\Omega^{+}$и $\Omega^{-}$с одинаковыми данными Неймана $\partial_{\nu_{a}} u^{+}=\partial_{\nu_{a}} u^{-}$как переводящий $\partial_{\nu_{a}} u^{ \pm}$в $u^{-}-u^{+}$. Этот оператор равен сумме $T_{\omega}^{+}+T_{\omega}^{-}$. Это Эллиптический ПДО порядка - 1 с эрмитовым отрищательно определенньм главньп символом, значит, с нулевьм индексом. Если он переводит некоторую функщию в нуль, то снова проверяется, что она равна нулю. Значит, оператор $T_{\omega}$ имеет обратньй - ПДО $\mathscr{T}_{\omega}$ порядка 1. Задача IV эквивалентна уравнению

$$
\mathscr{T}_{\omega} \varphi=\mu \varphi
$$

где $\varphi=u^{-}-u^{+}$, в смысле совпадения собственных функций (на $Г$ ) и собственных значений. Спектральные свойства оператора $\mathscr{T}_{\omega}$ аналогичны спектральным свойствам операторов $\mathscr{T}_{\omega}^{-}$и $\mathscr{T}_{\omega}^{+}+\mathscr{T}_{\omega}^{-}$.

4.3. Системы с постоянными коэффициентами и метод потенциалов. В этом пункте мы ограничимся рассмотрением системы вида

$$
\sum_{j, k} a_{j k} \partial_{j} \partial_{k} u(x)+\omega^{2} u(x)=0
$$

с постоянными вещественными коэффициентами $a_{j k}$, предполагая, что

$$
a_{j k}^{\prime}=a_{k j}
$$

Остальные предположения такие же, как в п. 4.1. В основном мы следуем здесь [9]. Система (4.24) является формально самосопряженной, если $\omega^{2}$ вещественно.

У соответствующего оператора $L_{0}$ (см. п. 1.1) спектр задачи Дирихле дискретен и положителен, а спектр задачи Неймана дискретен и неотрицателен, это легко проверяется при помощи формулы Грина. Поэтому однозначная разрешимость этих задач нарушается только для дискретного множества соответственно положительных и неотрицательных значений параметра $\omega$, стремящихся к $\infty$ (что, впрочем, верно и 
в предположениях п. 4.2). В частности, эти задачи однозначно разрешимы при невещественных $\omega$.

Фундаментальное решение $E_{\omega}(x)$ определено теперь глобально формулами, указанными в п. 4.1. Это четная по $x$ симметричная матрица. Используя его, введем поверхностные потенииалы простого и двойного слоя

$$
\mathscr{A}_{\omega} \varphi(x)=\int_{\Gamma} E_{\omega}(x-y) \varphi(y) d S_{y} \quad \text { и } \mathscr{B}_{\omega} \psi(x)=\int_{\Gamma}\left[\partial_{\nu_{a}(y)} E_{\omega}(x-y)\right]^{\prime} \psi(y) d S_{y} .
$$

В первой из этих формул $x$ любое, во второй $x \notin \Gamma$ и штрих обозначает транспонирование. Обозначим через $A_{\omega} \varphi(x)$ сужение первого из этих интегралов на $x \in \Gamma$ и через $B_{\omega} \psi(x)$ прямое значение второго интеграла на $\Gamma$ :

$$
A_{\omega} \varphi(x)=\int_{\Gamma} E_{\omega}(x-y) \varphi(y) d S_{y} \quad \text { и } \quad B_{\omega} \psi(x)=\int_{\Gamma}\left[\partial_{\nu_{a}(y)} E_{\omega}(x-y)\right]^{\prime} \psi(y) d S_{y}
$$

$(x \in \Gamma)$. Как будет видно дальше, введенный здесь оператор $A_{\omega}$ совпадает с оператором $A_{\omega}$ из предыдущего пункта (если система имеет вид (4.24)).

В теории ПДО есть описание ядер интегральных операторов, являюшихся псевдодифференциальньми операторами (см. [114] или формулировки в [4; п. 1.6]). Используя это описание, можно заново сделать вьвод, что $A_{\omega}-$ ПДО порядка -1 на $Г$. Поэтому $A_{\omega}$ - ограниченньй оператор из $H^{t}(\Gamma)$ в $H^{t+1}(\Gamma)$ при любом $t$. Его главный символ $\sigma_{A}$ вычисляется следуюшим образом. Зафиксируем точку на $\Gamma$, примем ее за начало координат и совместим координатную плоскость $x_{n}=0$ с касательной плоскостью в этой точке. Тогда в этой точке в координатах $\left(x^{\prime}, \xi^{\prime}\right)$, где, в частности, $\xi^{\prime}=\left(\xi_{1}, \ldots, \xi_{n-1}\right)$

$$
\sigma_{A}\left(0, \xi^{\prime}\right)=-\frac{1}{2 \pi} \int\left[a_{0}(\xi)\right]^{-1} d \xi_{n}
$$

(см. [11; п. 3.4]). Отсюда видно, что $A_{\omega}$ - эллиптический оператор, притом с нулевым индексом, так как главньй символ эрмитов и остается эрмитовым при поворотах системы координат. Ясно также, что этот символ отрицательно определен.

Оператор $B_{\omega}$ есть ПДО порядка -1 в случае уравнения Гельмгольца или Лапласа [1]. В общем случае это сингулярный интегральный оператор, понимаемьй в смысле главного значения по Коши. В частности, это так в случае системы Ламе (см. [12]). Иначе говоря, это ПДО нулевого порядка. Поэтому он действует ограниченным образом в пространстве $H^{t}(\Gamma)$ при любом $t$.

Операторы $\mathscr{A}_{\omega}$ и $\mathscr{B}_{\omega}$ - кограничные (см., например, [36], [69], [107]), первьй действует ограниченным образом из $H^{t}(\Gamma)$ в $H^{t+3 / 2}\left(\Omega^{+}\right)$и в $H^{t+3 / 2}\left(\Omega_{R}^{-}\right)$, а второй - из $H^{t}(\Gamma)$ в $H^{t+1 / 2}\left(\Omega^{+}\right)$и $H^{t+1 / 2}\left(\Omega_{R}^{-}\right)$при сколь угодно большом $R$.

Обозначим через $B_{\omega}^{\prime}$ оператор, транспонированный к $B_{\omega}$ :

$$
B_{\omega}^{\prime} \psi(x)=\int_{\Gamma}\left[\partial_{\nu_{a}(x)} E_{\omega}(x-y)\right] \psi(y) d S_{y} \quad(x \in \Gamma) .
$$

Это тоже ПДО на Г нулевого порядка. Следуюшие формулы хорошо известны (см., например, [40], [83]):

$$
\begin{gathered}
\left(\mathscr{A}_{\omega} \varphi\right)^{ \pm}=A_{\omega} \varphi, \quad\left(\mathscr{B}_{\omega} \psi\right)^{ \pm}=\left(B_{\omega} \pm \frac{1}{2} I\right) \psi \\
\left(\partial_{\nu_{a}} \mathscr{A}_{\omega} \varphi\right)^{ \pm}=\left(B_{\omega}^{\prime} \mp \frac{1}{2} I\right) \varphi, \quad\left(\partial_{\nu_{a}} \mathscr{B}_{\omega} \psi\right)^{+}=\left(\partial_{\nu_{a}} \mathscr{B}_{\omega} \psi\right)^{-} .
\end{gathered}
$$


В [9; пр. 3.1] с использованием первой формулы в (4.31) показано, что при условии II оператор $\frac{1}{2} I \pm B_{\omega}^{\prime}$ - эллиптический ПДО с главным символом (в той же, что и выше, системе локальных координат)

$$
\sigma_{\frac{1}{2} I \pm B_{\omega}^{\prime}}\left(0, \xi^{\prime}\right)=\frac{1}{2 \pi i} \oint_{\gamma_{ \pm}} \sigma_{\nu_{a}}\left(\xi^{\prime}, \tau\right)\left[a\left(\xi^{\prime}, \tau\right)\right]^{-1} d \tau
$$

здесь $\gamma_{ \pm}-$контуры в верхней и нижней полуплоскости, охватьваюшие все лежашие там корни определителя матрицы $a\left(\xi^{\prime}, \tau\right)$, а $\sigma_{\nu_{a}}-$ символ конормальной производной. Индекс этого ПДО равен нулю - это проверяется при помоши гомотопий. Как следствие такие же результаты верны для ПДО $\frac{1}{2} I \pm B_{\omega}$. Для уравнения Гельмгольца это тривиальные результаты, так как в этом случае $B_{\omega}^{\prime}$ имеет порядок -1 , для системы Ламе подсчет содержится в [70; гл. VI, §3] и [12], а для системы анизотропной упругости при $n=3-$ в [97], [98].

Теперь мы сформулируем наиболее сушественные утверждения в этом пункте, а затем приведем пояснения к их доказательствам. Первое - об интегральных представлениях решений в $\Omega^{ \pm}$через их данные Коши.

ТЕОрема 4.5. Для решений системы (4.24) в $\Omega^{ \pm}$, гладких вплоть до границы и удовлетворяющих нужным условиям на бесконечности в случае области $\Omega^{-}$, справедливы следующие интегральные представления через даннье Коши этих решений:

$$
u=\mathscr{B}_{\omega} u^{+}-\mathscr{A}_{\omega}\left(\partial_{\nu_{a}} u^{+}\right){ }_{\boldsymbol{\theta}} \Omega^{+} \quad u \quad u=\mathscr{A}_{\omega}\left(\partial_{\nu_{a}} u^{-}\right)-\mathscr{B}_{\omega} u^{-} \quad{ }_{\boldsymbol{\theta}} \Omega^{-} .
$$

Кроме того, правая часть первой из этих формул равна нулю в $\Omega^{-}$, а правая часть второй формуль - в $\Omega^{+}$.

Переходя в (4.33) на границу с учетом формул $(4.30),(4.31)$, получаем соотношения между данными Коши решений:

$$
\left(\frac{1}{2} I-B_{\omega}\right) u^{+}=-A_{\omega}\left(\partial_{\nu_{a}} u^{+}\right) \text {и }\left(\frac{1}{2} I+B_{\omega}\right) u^{-}=A_{\omega}\left(\partial_{\nu_{a}} u^{-}\right) .
$$

Следующее утверждение было предположением в предыдущем пункте.

Теорема 4.6. Для задач $D_{\omega}^{-}$и $N_{\omega}^{-}$nри всех допустимых $\omega$ имеет место единственность.

Третье утверждение - ключ к условиям обратимости операторов $A_{\omega}$ и $\frac{1}{2} I \pm B_{\omega}$.

ТеОремА 4.7. Справедливы следующие формуль:

$$
\begin{gathered}
\operatorname{Ker} A_{\omega}=\left\{\partial_{\nu_{a}} u^{+}: L_{\omega}(\partial) u=0{ }_{\boldsymbol{\theta}} \Omega^{+}, u^{+}=0\right\}=\operatorname{Ker}\left(B_{\omega}^{\prime}+\frac{1}{2} I\right), \\
\operatorname{Ker}\left(B_{\omega}-\frac{1}{2} I\right)=\left\{u^{+}: L_{\omega}(\partial) u=0{ }_{\boldsymbol{\theta}} \Omega^{+}, \partial_{\nu_{a}} u^{+}=0\right\} .
\end{gathered}
$$

Все это хорошо известно в случае уравнения Гельмгольца $[27 ; \S 3.4]$ и достаточно хорошо известно в случае системы Ламе $[70 ;$ гл. VII, $\S 2] .^{5}$ В общем случае вся картина очень близка к известной в этих классических случаях, но при $\omega>0$ немного сложнее доказательство второй формулы в (4.33) и существенно сложнее доказательство теоремы 4.6. Здесь мы пользуемся методикой работы с условиями излучения, разработанной в [121], [122] и приспособленной к граничным задачам для уравнений анизотропной упругости в [98]. Детали см. в [9].

\footnotetext{
${ }^{5}$ Первая формула в (4.35) получена в этих случаях в [1] и [12], см. также [9].
} 
ДоКАЗАТЕЛЬСТВО ТЕОРЕмЫ 4.5. Проверим сначала первую формулу при $x \in \Omega$ (подробнее, чем в [9]). До некоторого момента мы не будем пользоваться условием симметрии (4.25), и будет видно, что интегральное представление решений в $\Omega$ получается и без этого предположения, но с несколько более сложным потенциалом типа двойного слоя (ср. [83]).

При помощи интегрирования по частям проверяется формула Грина без комплексного сопряжения, вида

$$
\begin{gathered}
\int_{\Omega}\left[a\left(\partial_{y}\right)+\omega^{2}\right] u(y) \cdot v(y) d y-\int_{\Omega} u(y) \cdot\left[a^{\prime}\left(\partial_{y}\right)+\omega^{2}\right] v(y) d y \\
=\int_{\Gamma} \partial_{\nu_{a}} u^{+}(y) \cdot v^{+}(y) d S-\int_{\Gamma} u^{+}(y) \cdot \widetilde{\partial}_{\nu_{a}} v^{+}(y) d S
\end{gathered}
$$

где

$$
\widetilde{\partial}_{\nu_{a}} v^{+}(y)=\sum \nu_{k}(y) a_{j k}^{\prime} \partial_{j} v^{+}(y) .
$$

Применим формулу (4.37) к решению $u(x)$ системы (4.24) и столбцам фундаментального решения $E_{\omega}^{\prime}(y-x)$ транспонированной системы (с $a^{\prime}\left(\partial_{y}\right)$ вместо $\left.a\left(\partial_{y}\right)\right)$, где $x \in \Omega$. Точнее, удобно сначала вырезать окрестность $O_{\varepsilon}(x)$ точки $x$ радиуса $\varepsilon$ и потом перейти к пределу при $\varepsilon \rightarrow 0$. Получим представление строки $-u(x)^{\prime}$. Его можно переписать в виде представления для столбца $u(x)$ следуюшим образом:

$$
u(x)=-\int_{\Gamma} E_{\omega}(x-y) \partial_{\nu_{a}(y)} u^{+}(y) d S+\int_{\Gamma}\left(\widetilde{\partial}_{\nu_{a}(y)} E_{\omega}^{\prime}(x-y)\right)^{\prime} u^{+}(y) d S .
$$

Здесь справа первое слагаемое - потенщиал простого слоя, тот же, что в доказываемой формуле. Второе слагаемое обозначим через $\widetilde{\mathscr{B}}_{\omega} u^{+}$. Подробно оно записьвается так:

$$
\int_{\Gamma}\left[\sum \nu_{k}(y)\left(\partial_{j} E_{\omega}\right)(y-x) a_{j k}\right] u^{+}(y) d S .
$$

Очевидно, что это решение системы (4.24). Теперь вернемся к предположению, что вьполнено условие симметрии (4.25). Тогда $\widetilde{\partial}_{\nu_{a}}=\partial_{\nu_{a}}$ и, как нетрудно проверить, $\widetilde{\mathscr{B}}_{\omega}=\mathscr{B}_{\omega}$ (см. (4.26)). Первая формула в (4.33) доказана.

Аналогично получается вторая формула при невешественном $\omega$ и при $\omega=0, n \geqslant 3$. При этом формула Грина применяется в $\Omega_{R}^{-} \backslash O_{\varepsilon}(x)$, затем $\varepsilon$ устремляется к 0 , а $R$ к бесконечности. При $\omega>0$, как в [121], [122], надо проинтегрировать все члены в формуле Грина по $\Omega_{r}^{-}$по $r$ от $R$ до $2 R$, поделить их на $R$ и воспользоваться следуюшим вспомогательньм предложением (ср. [98]).

ПрЕДЛОЖЕНИЕ 4.8. Пусть $\omega>0 u v(x), w(x)-$ два решения системы (4.24) в окрестности бесконечности, удовлетворяющие условиям излучения. Тогда

$$
R^{-1} \int_{R}^{2 R} d r \int_{S_{r}} \partial_{\nu_{a}} v \cdot w d S \rightarrow 0 \quad n p u \quad R \rightarrow \infty
$$

əде $S_{r}=\{x:|x|=r\}$ и $\nu$ - внешняя нормаль $к$ этой сфере.

Это предложение доказывается с использованием соотношений (4.11). 
ДоКАЗАТЕЛЬСТВо ТЕОРЕМЫ 4.6. При невещественном $\omega$ и при $\omega=0, n \geqslant 3$ достаточно воспользоваться формулой Грина в $\Omega_{R}^{-}$. При $\omega>0$ проверяются и используются следующие два вспомогательных предложения (ср. [121], [122] и [98]).

ПРЕДЛОЖЕНИЕ 4.9. Пусть $\omega>0$ и и(x) - решение системы (4.1) в $\Omega^{-}$, удовлетворяющее условию

$$
\operatorname{Im} \int_{\Gamma} \partial_{\nu_{a}} u^{-} \cdot \bar{u}^{-} d S \leqslant 0 .
$$

Тогда $и(x)=O\left(|x|^{-(n+1) / 2}\right)$ nри $x \rightarrow \infty$.

В доказательстве при помоши формулы Грина проверяется, что в $(4.11) c_{p}(\alpha)$ при условии (4.41) равны нулю.

ПРЕДЛОЖЕНИЕ 4.10. При $\omega>0$ решение системы (4.24) в $\mathbb{R}^{n}$, удовлетворяющее условиям излучения, тождественно равно 0.

Последний факт постулировался в предыдущем пункте. Это предложение проверяется при помоши представления решения в фиксированной точке через данные Коши на сфере большого радиуса и предложения 4.8 .

Полное доказательство теоремы 4.6 см. в [9]. Она получается со следующим усилением, которое используется в доказательстве теоремы 4.13.

ТеОрема 4.11. При условии (4.41) решение системы (4.24) с $\omega>0$ в $\Omega^{-}$, удовлетворяющее условиям излучения, тождественно равно нулю.

ДОКАЗАТЕЛЬСТВо ТЕОРЕМЫ 4.7. Оно несложное, но существенно использует теорему 4.6. Проверим, например, первое равенство в (4.35).

Пусть $u(x)$ - нетривиальное решение однородной задачи $D_{\omega}^{+}$. Тогда $u \in C^{\infty}\left(\overline{\Omega^{+}}\right)$ ввиду эллиптичности задачи и первая из формул (4.34) показьвает, что $A_{\omega} \partial_{\nu_{a}} u^{+}=0$. Обратно, пусть $A_{\omega} \varphi=0$. Рассмотрим потенциал $u=\mathscr{A}_{\omega} \varphi$. Так как $u^{-}=0$, то $u$ - тождественньй нуль в $\Omega^{-}$по теореме 4.6 и, значит, $\partial_{\nu_{a}} u^{-}=0$. Теперь первые формулы в (4.31) показьвают, что $\partial_{\nu_{a}} u^{+}=\varphi$.

Теперь мы заново обсудим сведе́ние задач I-IV на Г. Для простоты будем предnолагать, что $\omega$ не исключительно относительно задач $D_{\omega}^{+} u N_{\omega}^{+}$, т.е. для них имеет место единственность и, следовательно, однозначная разрешимость. Это равносильно обратимости операторов $A_{\omega}$ (в смысле существования обратного ПДО порядка 1), $\frac{1}{2} I \pm B_{\omega}$ и $\frac{1}{2} \pm B_{\omega}^{\prime}$.

Прежде всего отметим, что поверхностные потенциалы можно использовать для явного решения задач Дирихле и Неймана. Например, решение задачи $D_{\omega}^{+}$строится по хорошо известным формулам

$$
u=\mathscr{A}_{\omega} A_{\omega}^{-1} u^{+}=\mathscr{B}_{\omega}\left(B_{\omega}+\frac{1}{2} I\right)^{-1} u^{+} .
$$

Теперь мы полагаем

$$
\mathscr{T}_{\omega}^{ \pm}=A_{\omega}^{-1}\left(\frac{1}{2} I \mp B_{\omega}\right), \quad T_{\omega}^{ \pm}=\left(\mathscr{T}_{\omega}^{ \pm}\right)^{-1}=\left(\frac{1}{2} I \mp B_{\omega}\right)^{-1} A_{\omega} .
$$

Как легко видеть, это операторы (4.15), (4.20) и обратные к ним операторы для системы (4.24). С ними, как мы знаем, задачи I и II сводятся соответственно к уравнениям 
(4.18) и (4.21), если рассматривать только собственные функции. Имея эти операторы, мы умеем сводить к уравнениям на $Г$ и остальные задачи III и IV. Задача III эквивалентна уравнению

$$
\mu A_{\omega} \psi=\psi
$$

с $\psi=\partial_{\nu_{a}} u^{-}-\partial_{\nu_{a}} u^{+}$, которое, в свою очередь, эквивалентно уравнению (4.22).

Отметим еше две полезных формулы. Первая из них имеет вид

$$
B_{\omega} A_{\omega}=A_{\omega} B_{\omega}^{\prime}
$$

Она при $\omega^{\prime}>0$ получается сравнением двух выражений для $\partial_{\nu_{a}} u^{+}$через $u^{+}$, вытекающих из (4.15), (4.43) и первых формул в (4.42), (4.31). Затем она предельньпм переходом распространяется на все допустимые $\omega$. Вторая формула имеет вид

$$
\mathscr{T}_{\omega} \varphi=-\left(\partial_{\nu_{a}} B_{\omega} \varphi\right)^{ \pm}
$$

это так называемый гиперсингулярный оператор теории потенциала. Доказательство см., например, в [9; пр. 4.2].

Главньй символ ПДО $\mathscr{T}_{\omega}^{ \pm}$в каждой граничной точке получается перемножением главных символов ПДО $A_{\omega}^{-1}$ и $\frac{1}{2} I \mp B_{\omega}$.

В случае постоянных коэффициентов можно получить дополнительную информацию о спектральных свойства операторов, отвечающих задачам I-IV, связанную со структурой системы (4.24). Мы покажем это на примере задачи III. Для ясности сначала полнее, чем ранњше, опишем структуру фундаментального решения $E_{\omega}(x)$ при $\omega>0$.

Его исследование начинается с проверки того, что матрица $[a(\xi)-\vartheta I]^{-1}$ при условии $\mathrm{V}$ допускает представление $M^{-1}(\xi, \vartheta) l(\xi, \vartheta)$, где $M(\xi, \theta)$ - минимальньй многочлен матрицы $a(\xi)$, равный произведению всех разностей $\vartheta-d_{p}(\xi)(p=1, \ldots, q)$, а элементы матрицы $l(\xi, \vartheta)$ однородны по $(\xi, \vartheta)$. Далее, $M\left(\xi, \omega^{2}\right)$ - многочлен от $\xi$, которьй разлагается в произведение неприводимых множителей, полиномиальных и однородных по $(\xi, \omega)$. Каждый такой многочлен имеет вещественные нули - некоторые из поверхностей $S_{p}$, и на них его градиент отличен от нуля. См. [9].

Зафиксируем положительное $\omega$. Пусть все вешественные нули многочлена $M\left(\xi, \omega^{2}\right)$ лежат в шаре $\{\xi:|\xi|<\rho\}$. Возьмем скалярную вешественную функцию $h_{0}(\xi) \in$ $C_{0}^{\infty}\left(\mathbb{R}^{n}\right)$, зависящую только от $|\xi|$, равную 1 при $|\xi|<2 \rho$, и положим $h_{1}(\xi)=1-h_{0}(\xi)$. Результат вычисления предела (4.9) оказывается следуюшим.

ПРЕДЛОЖЕНИЕ 4.12. При $\omega>0$

$$
E_{\omega}(x)=E_{\omega}^{(1)}(x)+E_{\omega}^{(2)}(x)+E_{\omega}^{(3)}(x),
$$

где

$$
\begin{aligned}
& E_{\omega}^{(1)}(x)=\frac{1}{(2 \pi)^{n}} \mathrm{p} \cdot \mathrm{v} \cdot \int e^{i x \cdot \xi} h_{0}(\xi) M^{-1}\left(\xi, \omega^{2}\right) l\left(\xi, \omega^{2}\right) d \xi \\
& E_{\omega}^{(2)}(x)=\frac{i \pi}{(2 \pi)^{n}} \sum_{1}^{q}(-1)^{q-p-1} \int_{S_{p}} e^{i x \cdot \xi}\left|\operatorname{grad}_{\xi} M\left(\xi, \omega^{2}\right)\right|^{-1} l\left(\xi, \omega^{2}\right) d S \\
& E_{\omega}^{(3)}(x)=\frac{1}{(2 \pi)^{n}} \int e^{i x \cdot \xi} h_{1}(\xi) M^{-1}\left(\xi, \omega^{2}\right) l\left(\xi, \omega^{2}\right) d \xi
\end{aligned}
$$


Здесь в (4.48) p.v. - главное значение интеграла по Коши (предел интеграла по $\left\{\xi:\left|M\left(\xi, \omega^{2}\right)\right|>\delta\right\}$ при $\left.\delta \rightarrow 0\right)$. Интеграл в (4.50) обычным образом регуляризуется. Эта часть $E_{\omega}^{(3)}(x)$ фундаментального решения определяет его особенность в $x=0$, аналитична при $x \neq 0$ и быстро убьвает на бесконечности, а $E_{\omega}^{(1)}(x)$ и $E_{\omega}^{(2)}(x)$ - аналитические функции, асимптотика которых на бесконечности вычисляется методом стационарной фазы и определяет указанную вьше асимптотику фундаментального решения.

Теперь сформулируем теорему о спектральных свойствах оператора $A_{\omega}$. Переход к обратному оператору не составляет труда.

Теорема 4.13. Оператор $A_{\omega}$ обладает комплексной симметрией: $A_{\omega}^{\prime}=A_{\omega}$ при всех допустимых $\omega$. Это компактный оператор в любом пространстве $H^{t}(\Gamma)$ с собственными или корневыми функичями, принадлежсащими $C^{\infty}(\Gamma)$.

При $\omega^{\prime}=0$ он является самосопряженным оператором в $L_{2}(\Gamma)$ и из его собственных функиий можсно составить ортонормированный базис в $H^{0}(\Gamma)$, остающийся безусловным базисом во всех пространствах $H^{t}(\Gamma)$.

При остальных $\omega$ оператор $A_{\omega}$ есть слабое возмущение самосопряженного оператора, а именно $\operatorname{Re} A_{\omega}$. Более точно, $A_{\omega}-\bar{A}_{\omega}$ - оператор порядка -3 в соболевской шкале $H^{t}(\Gamma)$, если $\omega^{\prime} \omega^{\prime \prime} \neq 0$, и порядка $-\infty$, если $\omega>0$. Из корневьх функиий можно составить систему, являюшуюся безусловныл базисом со скобками во всех $H^{t}(\Gamma)$, если $n \leqslant 3$ и/ или $\omega>0$, и базис для метода суммирования Абеля-Лидского любого порядка $\alpha>n-3$, если $\omega^{\prime} \omega^{\prime \prime} \neq 0$ u $n>3$.

Характеристические числа $\mu_{j}$ оператора $A_{\omega}$ имеют асимптотику вида $(4.19)$ с положительной постоянной $C_{n}$, зависящей только от главного символа.

Если $\omega^{\prime} \neq 0$, то оператор $\pm A_{\omega}$ диссипативен при $\mp \omega^{\prime}>0$. Более того, справедливо неравенство ${ }^{6} \omega^{\prime} \operatorname{Im}\left(A_{\omega} \varphi, \varphi\right)_{\Gamma}<0$ при $A_{\omega} \varphi \neq 0$. Поэтому знаки мнимых частей $\operatorname{Im} \mu_{j}$ совпадают со знаком $\omega^{\prime}$. При этом $\operatorname{Im} \mu_{j}=O\left(j^{-h}\right)$, әде $h=1 /(n-1)$ при $\omega^{\prime} \omega^{\prime \prime} \neq 0$ и $h$ сколь угодно велико при $\omega>0$.

Действительные части $\operatorname{Re} \mu_{j}$ отричательньи при $\left|\omega^{\prime}\right| \leqslant \omega^{\prime \prime}$.

Здесь достаточно пояснить, как получаются дополнительные свойства оператора $\mathscr{A}_{\omega}$ (по сравнению с отмеченными в предыдущем пункте).

Из формул для фундаментального решения в п. 4.1 и предложения 4.12 можно усмотреть, что оператор $A_{\omega}$ имеет симметричное ядро и отличается от своей действительной части на слагаемое порядка не выше -3 , т.е. разнища порядков не меньше 2 . Если же $\omega>0$, то самосопряженности нет, но разница порядков оказывается бесконечной, т.е. в этом случае оператор $A_{\omega}$ - бесконечно слабое возмущение самосопряэсенного оператора. Хороший пример, на котором это совсем ясно видно, - уравнение Гельмгольца (1.21). В последнем случае ядро интегрального оператора $\operatorname{Im} A_{\omega}$ пропорционально $\sin (\omega|x-y|) /|x-y|$, т.е. это бесконечно гладкая функция. По поводу дополнительных спектральных свойств, которыми обладают операторы, столь близкие к самосопряженным, мы отсылаем читателя к [4]. В формулировке мы отметили только быстрое убывание мнимых частей характеристических чисел. Диссипатив-

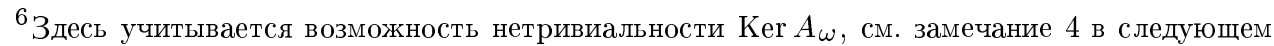
пункте.
} 
ность несамосопряженного оператора $A_{\omega}$ или $-A_{\omega}$ проверяется при помоши формулы Грина.

Для операторов $T_{\omega}^{ \pm}$и $T_{\omega}$ получаются такие же теоремы, но $T_{\omega}^{+}$является самосопряженньм оператором также при $\omega>0$.

\section{4. Замечания о предположениях в двух предыдущих пунктах.}

1. Поверхностные потенциалы можно ввести и использовать и в случае переменных коэффициентов. Достаточно предположить, что глобально сушествует фундаментальное решение $E(x, y)$ (хотя в принципе можно работать и с параметриксом). Оно имеет такую же особенность при $x=y$, как $E_{\omega}(x-y)$, принадлежит $C^{\infty}$ при $x \neq y$, если коэффициенты бесконечно гладкие, и аналитично, если коэффициенты аналитичны. Сp. [91], [92].

2. Если $\omega$ таково, что для задачи $D_{\omega}^{+}$или $N_{\omega}^{+}$нет единственности, а коэффициенты (для простоты) постоянны, то оператор $\mathscr{T}_{\omega}^{-}$по-прежнему существует и обратим. Его явное представление можно построить, используя некоторые модификации поверхностных потенциалов, см., например, $[27 ; \S 3.6],[9 ;$ п. 5.4] и приведенные там ссылки.

3. Если при данном $\omega$ нет единственности для внутренней задачи Неймана, то эту задачу можно заменить в наших рассмотрениях однозначно разрешимой задачей с граничным условием $\partial_{\nu_{a}} u^{+}+\sigma u^{+}=g$, подобрав подходяшее $\sigma$. На этом тоже не будем останавливаться, см. [9] и приведенные там ссылки.

4. Отметим еще следующую возможность экономии в предположениях. Оператор $A_{\omega}$ определен при всех допустимых $\omega$, но $A_{\omega}^{-1}$ не сушествует, если нет единственности для задачи $D_{\omega}^{+}$. Однако в этом случае собственное подпространство оператора $A_{\omega}$, отвечающее нулевому собственному значению, конечномерно, и оно состоит из бесконечно гладких функций: то и другое следует из эллиптичности этого оператора. Пусть коэффициенты в системе постоянны; тогда конечномерно соответствующее корневое подпространство, оно просто совпадает с собственным подпространством, так как присоединенных функций в нем нет ввиду диссипативности оператора $-A_{\omega}$. Это позволяет заменить нулевое собственное значение ненулевым без изменения корневых функций и остальных собственных значений. После этого обратньй ПДО (порядка 1 ) уже сушествует. Поэтому при нарушении единственности для задачи $D_{\omega}^{+}$оператор $A_{\omega}$ по сушеству сохраняет свои спектральные свойства, указанные в теореме 4.13.

5. Вместо задач в областях $\Omega^{ \pm}$пространства $\mathbb{R}^{n}$ можно рассматривать задачи в дополнительных одна к другой областях $\Omega^{ \pm}$на (компактном) замкнутом многообразии. Тогда отпадает необходимость в условиях излучения и легко указываются условия, достаточные для единственности решения системы на этом многообразии и для единственности решений внешних задач Дирихле и Неймана. (Можно предположить, что система содержит большой параметр.) См., в частности, работы [89], [91], [92] о задачах Дирихле и Неймана в липшицевой области на замкнутом многообразии.

4.5. Кулоновские особенности и ослабление предположений о гладкости. Если включить в систему добавочное слагаемое - мультипликатор из $H^{1}(\Omega)$ в $H^{0}(\Omega)$, то, как мы знаем из $\S 1$, при подходяших значениях параметра в системе внутренние задачи Дирихле и Неймана однозначно разрешимы и можно строить решения в $H^{2}(\Omega)$. В этом случае оператор $\mathscr{T}_{\omega}^{+}$можно рассматривать как обратимьй оператор 
из $H^{t+1}(\Gamma)$ в $H^{t}(\Gamma)$, по крайней мере, при $0 \leqslant t \leqslant 1 / 2$ (на самом деле этот промежуток можно продлить влево, см. $\S 5)$. При наличии формальной самосопряженности оператор $\mathscr{T}_{\omega}^{+}$остается самосопряженньм в $H^{0}(\Gamma)$ и ортонормированный базис из его собственных функций в $H^{0}(\Gamma)$ остается безусловным базисом в $H^{t}(\Gamma)$ при $0<t \leqslant 3 / 2$. Если $n=2$ или $n=3$, то сохраняется равномерная сходимость рядов Фурье по этим собственньм функциям для функций соответственно из $C^{1}(\Gamma)$ или $C^{2}(\Omega)$. Сохраняется также асимптотическая формула (4.19) для собственных значений с оптимальной оценкой остатка. Последнее следует из сказанного в пш. 2.8 и 3.2.

Только что указанные утверждения о базисности собственных функций сохранятся также, если мы ослабим предположения о гладкости до предположений из п. 1.5.

\section{$\S 5$. Задачи со спектральным параметром на липшицевой границе}

5.1. Вариационный подход к системам с переменными коэффициентами. В этом пункте мы снова рассматриваем систему (4.1) в $\mathbb{R}^{n}, n \geqslant 2$, предполагая, что при $|x|>R$ с достаточно большим $R$ она принимает вид (4.24). Граница $\Gamma$ - произвольная связная (для простоты) липшицева поверхность. Предположения о гладкости коэффициентов - такие же, как в п. 1.5. Система подчинена условиям сильной эллиптичности, симметрии (1.3) и II. Как и в $\S 4, \omega=\omega^{\prime}+i \omega^{\prime \prime}$ и либо $\omega^{\prime}>0$, либо $\omega \geqslant 0$. Формальную самосопряженность мы понимаем в смысле, указанном в начале п. 4.1.

Задачи I-IV поставлены в п. 4.1. В задачах II-IV решение подчиняется сформулированным там условиям на бесконечности. Для этого, если $\omega>0$, система при $|x|>R$ с достаточно большим $R$ подчиняется условию V. Случай $n=2, \omega=0$ в задачах II-IV исключается из рассмотрения, хотя можно было бы и не делать этого (ср. [123] и [87]).

Задача I. Основу для ее рассмотрения составляют результаты по задачам Дирихле и Неймана из $\S 2$ и $\S 3$. Мы предположим, что для этих задач при данном $\omega$ имеет место единственность, или, что то же, однозначная разрешимость. Тогда снова, как в п. 4.2, вводится оператор $\mathscr{T}_{\omega}^{+}$. Мы уже не можем рассматривать его как эллиптический ПДО. Однако мы знаем, что по $u^{+} \in H^{t}(\Gamma)$ при $0<t<1$ однозначно строится решение $u \in H^{t+1 / 2}(\Omega)$ однородной системы и для него определено значение $\partial_{\nu_{a}} u^{+}$, принадлежашее $H^{t-1}(\Gamma)$. И наоборот, по $\partial_{\nu_{a}} u^{+} \in H^{t-1}(\Gamma)$ однозначно восстанавливается $u^{+} \in H^{t}(\Gamma)$. Таким образом, $\mathscr{T}_{\omega}^{+}-$ограниченный обратимый оператор из $H^{t}(\Gamma)$ в $H^{t-1}(\Gamma), t \in(0,1)$. Обратньй оператор мы обозначаем через $T_{\omega}^{+}$. Задача I на собственные функции эквивалентна уравнению $(4.18)$ с $\varphi=u^{+}$.

Пусть сначала система формально самосопряженная. Тогда остается в силе соотношение (4.16) при $u \in H^{t}(\Gamma), v \in H^{1-t}(\Gamma)(0<t<1)$ (при этом имеется в виду соответствуюшее продолжение скалярного произведения в $\left.H^{0}(\Gamma)\right)$. К сожалению, мы не вправе рассматривать оператор $T_{\omega}^{+}$или $\mathscr{T}_{\omega}^{+}$в $H_{0}(\Gamma)$. Но

$$
\left(T_{\omega}^{+} \varphi, \psi\right)_{\Gamma}=\left(\varphi, T_{\omega}^{+} \psi\right)_{\Gamma}
$$

в частности, при $\varphi, \psi \in H^{-1 / 2}(\Gamma)$. Это позволяет, как мы сейчас увидим, рассматривать $T_{\omega}^{+}$как самосопряженный оператор в $H^{-1 / 2}(\Gamma)\left(\right.$ вместо $\left.H^{0}(\Gamma)\right)$. Справедливо следуюшее предложение [9; пр. 7.10].

ПРЕДЛОЖЕНИЕ 5.1. Пусть $K$ - ограниченный обратимый оператор из $H^{-1 / 2}(\Gamma)$ в $H^{1 / 2}(\Gamma)$, самосопряжсенный в смысле

$$
(K \varphi, \psi)_{\Gamma}=(\varphi, K \psi)_{\Gamma}
$$


и пусть все собственные значения оператора $K$ положительны. Тогда норма в $H^{-1 / 2}(\Gamma)$ (как пространстве, сопряжсенном $\left.\kappa H^{1 / 2}(\Gamma)\right)$, умноженная на $\|K\|^{1 / 2}$, совпадает с выражением $(K \varphi, \varphi)^{1 / 2}$.

Это предложение позволяет принять за скалярное произведение в $H^{-1 / 2}(\Gamma)$ выражение $(5.2)$ с $K=\left|T_{\omega}^{+}\right|$(последний оператор получается из оператора $T_{\omega}^{+}$заменой всех его собственных значений их модулями). Так как оператор $T_{\omega}^{+}$перестановочен с $\left|T_{\omega}^{+}\right|$, то $T_{\omega}^{+}$оказьвается самосопряженным оператором в $H^{-1 / 2}(\Gamma)$ относительно этого скалярного произведения. Следовательно, он имеет там ортонормированньй базис из собственных функций. Они принадлежат $H^{t}(\Gamma)$ при $|t|<1$.

Если $\left\{\varphi_{l}\right\}$ - ортогональньй базис в $H^{-1 / 2}(\Gamma)$ из собственных функций с обычной нормировкой $\left\|\varphi_{l}\right\|_{0, \Gamma}=1$, то, как нетрудно проверить, разложения $\Phi$ урье в $H^{-1 / 2}(\Gamma)$ имеют обычньй вид

$$
\varphi=\sum\left(\varphi, \varphi_{l}\right)_{\Gamma} \varphi_{l}
$$

Сформулируем теперь аналог теоремы 4.1 для липшицевых поверхностей.

Теорема 5.2. Пусть задачи Дирихле и Неймана в $\Omega$ для системь (4.1) однозначно разрешимы. Тогда $\mathscr{T}_{\omega}^{+}-$ограниченный оператор из $H^{t}(\Gamma)$ в $H^{t-1}(\Gamma)$ при $0<t<1$, имеющий обратный $T_{\omega}^{+}$. Если система формально самосопряжсенная, то это самосопряженный оператор в $H^{-1 / 2}(\Gamma)$ относительно скалярного произведения (5.2) с $K=\left|T_{\omega}^{+}\right|$. Его собственные функиии принадлежсат $H^{t}(\Gamma)$ при $|t|<1$, и ортонормированный базис из них в $H^{-1 / 2}(\Gamma)$ остается безусловным базисом в этих пространствах. Собственные значения удовлетворяют двусторонней оценке

$$
0<C_{1} \leqslant\left|\mu_{l}\right| l^{-1 /(n-1)} \leqslant C_{2} .
$$

Если формальной самосопряэсенности нет, то $\mathscr{T}_{\omega}^{+}-$слабое возмущение самосопряженного оператора. В этом случае корневые функиии принадлежат $H^{t}(\Gamma)$ при $|t|<1$ и образуют базис для метода суммирования Абеля-Лидского любого порядка $r>n-2$ в каждом из этих пространств. Собственные значения удовлетворяют оценке $\left|\mu_{l}\right| \geqslant C l^{1 /(n-1)}$, и их действительные части стремятся $\kappa-\infty$. При этом $\left|\operatorname{Im} \mu_{l}\right| \leqslant C_{\varepsilon}^{\prime}\left|\operatorname{Re} \mu_{l}\right|^{\varepsilon}$ при больиих l, где $\varepsilon-$ сколь угодно малое положстельное число.

ДокАЗАТЕЛЬСтво. Нам осталось объяснить следующее.

Безусловная базисность собственных функций проверяется следуюшим образом. При $0<\theta<1$ степени $\left|T_{\omega}^{+}\right|^{\theta}$ самосопряженного оператора $\left|T_{\omega}^{+}\right|$изоморфно отображают $H^{-1 / 2}(\Gamma)$ на интерполящионное пространство $\left[H^{-1 / 2}(\Gamma), H^{1 / 2}(\Gamma)\right]_{\theta}$, совпадающее с $H^{\theta-1 / 2}(\Gamma)$. Это дает безусловную базисность в $H^{t}(\Gamma)$ для $|t|<1 / 2$, включая $t=0$. На остальные $t, 1 / 2 \leqslant|t|<1$, результат переносится при помоши изоморфизмов, определяемых операторами $T_{\omega}^{+}$и $\mathscr{T}_{\omega}^{+}$.

Соотношение (4.17) сохраняется в случае формально самосопряженной системы с $c=0$ и чисто мнимым $\omega$. Собственные значения в этом случае отрищательны. Отсюда следует, что всегда собственные значения или их действительные части стремятся $\mathrm{K}-\infty$.

Односторонняя оценка для $\left|\mu_{l}\right|$ следует из ограниченности $T_{\omega}^{+}$как оператора из $H^{-1 / 2}(\Gamma)$ в $H^{1 / 2}(\Gamma)$ (см. предпоследний абзац в п. 3.2). 
Объясним, как получается двусторонняя оценка для собственных значений в случае формальной самосопряженности. Пусть $T$ - любой ограниченный обратимьй оператор из $H^{-1 / 2}(\Gamma)$ в $H^{1 / 2}(\Gamma)$, и пусть $\widetilde{\Gamma}$ - бесконечно гладкая замкнутая поверхность, связанная с Г липшицевым диффеоморфизмом. (Такие поверхности существуют, подробнее об этом мы скажем в следующем пункте.) Используя его, получаем на $\widetilde{\Gamma}$ подобньй $T$ оператор $\widetilde{T}$, являюшийся ограниченным обратимым оператором из $H^{-1 / 2}(\widetilde{\Gamma})$ в $H^{1 / 2}(\widetilde{\Gamma})$. С другой стороны, на $\widetilde{\Gamma}$ есть самосопряженный эллиптический ПДО $\widehat{T}$ порядка -1 с положительными собственньми значениями, он тоже ограничен и обратим как оператор из $H^{-1 / 2}(\widetilde{\Gamma})$ в $H^{1 / 2}(\widetilde{\Gamma})$. Собственные значения оператора $\widehat{T}$ совпадают с его $s$-числами $s_{l}(\widehat{T})$ и имеют правильную асимптотику $s_{l}(\widehat{T}) \sim c l^{-1 /(n-1)}, c>0$. Так как $\widetilde{T}=\widehat{T}\left(\widehat{T}^{-1} \widetilde{T}\right)$ и $\widehat{T}=\widetilde{T}\left(\widetilde{T}^{-1} \widehat{T}\right)$ и здесь операторы в скобках ограничены, то $s_{l}(\widetilde{T}) \leqslant C^{(1)} s_{l}(\widehat{T})$ и $s_{l}(\widehat{T}) \leqslant C^{(2)} s_{l}(\widetilde{T})$. Это дает для $s_{l}(\widetilde{T})$ двустороннюю оценку $0<C^{(3)} \leqslant s_{l}(\widetilde{T}) l^{1 /(n-1)} \leqslant C^{(4)}$. Как следствие получается двусторонняя оценка для $s$-чисел оператора $T$ :

$$
0<C^{(5)} \leqslant s_{l}(T) l^{1 /(n-1)} \leqslant C^{(6)} .
$$

В частности, если $T$ - самосопряженный оператор, то это оценка для модулей его собственных значений. Отсюда следует нужньй результат для $\mathscr{T}_{\omega}^{+}$.

Пусть система не является формально самосопряженной. Тогда решение $u$ задачи Дирихле для однородной системы (4.1) с заданным значением $u^{+}$можно представить в виде суммы $u^{(0)}+u^{(1)}$, где $u^{(0)}-$ решение задачи Дирихле для однородной формально самосопряженной системы с тем же граничным значением $u^{+}$, а $u^{(1)}$ - решение неоднородной системы с некоторой правой частью $f$ и однородным граничньм условием Дирихле. Пусть $u^{+} \in H^{t}(\Gamma)$ с малым положительным $t$. Тогда $u^{(0)} \in H^{t+1 / 2}(\Omega)$ и $\partial_{\nu_{a}} u^{(0)+} \in H^{t-1}(\Gamma)$. Оператор $u^{+} \mapsto \partial_{\nu_{a}} u^{(0)+}$ обозначим через $\mathscr{T}_{\omega}^{(0)+}$. Он имеет в $H^{t-1}(\Gamma)$ безусловный базис из собственных функций и вещественные собственные значения, так что мы можем рассматривать его как самосопряженный оператор в этом пространстве с областью определения $H^{t}(\Gamma)$. Далее, $f$ получается применением дифференциального оператора порядка не вьше 1 к $u^{(0)}$ и поэтому принадлежит $H^{t-1 / 2}(\Omega)$. Следовательно, $u^{(1)} \in H^{3 / 2-\tau}(\Omega)$ со сколь угодно малым положительным $\tau$, это максимальная гладкость функции $u^{(1)}$, которую мы можем гарантировать. Далее, $\partial_{\nu_{a}} u^{(1)+} \in H^{-\tau}(\Gamma)$. Обозначим через $\mathscr{T}_{\omega}^{(1)+}$ оператор, переводящий $u^{+}$ в $\partial_{\nu_{a}} u^{(1)+}$. Этот оператор действует ограниченным образом из $H^{t}(\Gamma)$ в $H^{-\tau}(\Gamma)$. Таким образом, оператор $\mathscr{T}_{\omega}^{+}$“первого порядка" получается из самосопряженного оператора $\mathscr{T}_{\omega}^{(0)+}$ тоже "первого порядка" прибавлением оператора $\mathscr{T}_{\omega}^{(1)+}$ “сколь угодно малого положительного порядка". Это приводит к утверждениям об оценке мнимых частей собственных значений и о базисности для метода Абеля-Лидского в пространстве $H^{t-1}(\Gamma)$, указанным в формулировке теоремы. На остальные пространства последний результат распространяется при помощи известных нам изоморфизмов.

Задача II. Прежде всего заметим, что решения нашей однородной системы аналитичны при $|x|>R$. Рассматривая решения в $\Omega_{R^{\prime}}^{-}$с $R^{\prime}>R$, мы можем пользоваться теоремами о гладкости решений, доказанными в $\S 2$. Пространства, в которых мы можем рассматривать задачи Дирихле и Неймана в $\Omega^{-}$, остаются такими же при невещественном и нулевом $\omega$ и локально такими же при $\omega>0$. Предположим, что 
для задач $D_{\omega}^{-}$и $N_{\omega}^{-}$имеет место единственность и, следовательно, однозначная разрешимость. Тогда снова вводится оператор $\mathscr{T}_{\omega}^{-}$по формуле (4.20). Как и $\mathscr{T}_{\omega}^{+}$, он действует ограниченным образом из $H^{t}(\Gamma)$ в $H^{t-1}(\Gamma)$ при $0<t<1$ и имеет ограниченный обратньй - оператор $T_{\omega}^{-}$. Задача II на собственные функции эквивалентна уравнению (4.21) с $\varphi=u^{-}$. Если система формально самосопряженная и $\omega^{\prime}=0$, то оператор $T_{\omega}^{-}$является самосопряженньм в $H^{-1 / 2}(\Gamma)$ относительно скалярного произведения (5.2) с $K=\left|T_{\omega}^{-}\right|$. Он имеет там ортонормированньй базис из собственных функций, остаюшийся безусловным базисом в $H^{t}(\Gamma)$ при $|t|<1$.

Мы могли бы сформулировать теорему о спектральных свойствах оператора $\mathscr{T}_{\omega}^{-}$, но это было бы почти дословное повторение теоремы 5.2.

Задача III. Все аналогично только что сказанному про задачу II. Как в $\S 4, n p e д-$ полагаем, что внутренние и внешние задачи Дирихле и Неймана однозначно разрешимы и что решение однородной системы, удовлетворяющее условиям на бесконечности из $n$. 4.1, тождественно равно нулю. Задача III на собственные функции эквивалентна уравнению $(4.22)$ с $\varphi=u^{ \pm}$. Оператор $\mathscr{T}_{\omega}^{+}+\mathscr{T}_{\omega}^{-}$действует ограниченным образом из $H^{t}(\Gamma)$ в $H^{t-1}(\Gamma)$ при $0<t<1$ и имеет ограниченный обратньй - оператор $A_{\omega}$. Если система формально самосопряженная и $\omega^{\prime}=0$, то этот оператор является самосопряженным в $H^{-1 / 2}(\Gamma)$ относительно скалярного произведения (5.2) с $K=\left|A_{\omega}\right|$. Ортонормированный базис из его собственных функщий в этом пространстве остается тогда безусловным базисом в $H^{t}(\Gamma)$ при $|t|<1$. И в остальном спектральные свойства оператора $\mathscr{T}_{\omega}^{+}+\mathscr{T}_{\omega}^{-}$аналогичны спектральным свойствам оператора $\mathscr{T}_{\omega}^{-}$.

Задача IV. Делаем такие же предположения, как при рассмотрении задачи III. Задача IV на собственные функции эквивалентна уравнению (4.23) с $\varphi=$ $u^{-}-u^{+}$. Здесь $\mathscr{T}_{\omega}-$ оператор, обратньй к $T_{\omega}=T_{\omega}^{+}+T_{\omega}^{-}$. Оператор $\mathscr{T}_{\omega}$ действует ограниченным и обратимьм образом снова из $H^{t}(\Gamma)$ в $H^{t-1}(\Gamma)$ при $0<t<1$. Если система формально самосопряженная и $\omega^{\prime}=0$, то $T_{\omega}$ - самосопряженньй оператор в $H^{-1 / 2}(\Gamma)$ относительно скалярного произведения (5.2) с $K=\left|T_{\omega}\right|$. Ортонормированный базис из его собственных функций остается безусловным базисом в $H^{t}(\Gamma)$ при $|t|<1$. И в остальном спектральные свойства оператора $\mathscr{T}_{\omega}$ тоже аналогичны спектральным свойствам оператора $\mathscr{T}_{\omega}^{-}$.

В [28; т. 2] отмечена “сильная эллиптичность" операторов типа наших $A_{\omega}$ и $\mathscr{T}_{\omega}$ на липшицевой поверхности. Ср. [29].

Результатынастоящего пункта остаются справедливыми при включении в систему кулоновского потенциала. Более точно, в систему можно включить слагаемое $c_{2} u$, где $c_{2}(x)$ - эрмитова матрица и мультипликатор из $H^{1}(\Omega)$ в $H^{0}(\Omega)$. Это следует из сказанного в п. 2.9. Мы не будем рассматривать здесь более общие особенности.

5.2. Системы с постоянными коэффициентами и метод потенциалов. Здесь мы будем рассматривать систему (4.24). Поверхностные потенциалы $\mathscr{A}_{\omega}$ и $\mathscr{B}_{\omega}$, как и в случае гладкой поверхности, вводятся формулами (4.26), и соответствуюшие операторы на Г определяются формулами (4.27). Их можно использовать для исследования задач I-IV на основе результатов из $\S 2$ и $\S 3$, полученньх вариащионным методом. Но сейчас мы будем говорить об относительно новом подходе к исследованию граничных задач в липшицевых областях при помоши потенциалов. Он сформировался в конце $70-\mathrm{x}$ - начале $80-\mathrm{x}$ гг. в значительной степени в работах специалис- 
тов по гармоническому анализу. Све́дения об истории развития этого подхода можно найти, в частности, в обзорах Джерисона-Кёнига [52] и Кёнига [59], [60], а также в монографии [61], содержашей обширную литературу. Во многих работах отмечается сушественное влияние “программы Кальдерона" на эти исследования, см., например, [89]. Кальдерон предвидел, что исследование краевых задач для эллиптических уравнений в липшищевых областях будет продвинуто при помоши сингулярных интегральных операторов награницах этих областей. Поверхности класса $C^{1}$ рассмотрены в [38].

Этот подход в его ньнешней форме основан прежде всего на теореме об ограниченности сингулярного интегрального оператора в $L_{2}(\Gamma)$ на липшицевой поверхности $Г$. Эта теорема была доказана сначала для поверхностей с мальми липшицевыми константами и, в частности, для поверхностей класса $C^{1}$ в работе Кальдерона [24], а затем в общем случае в работе Койфмана, Макинтоша и Мейера [26]. Ключевым является случай интегралов типа Коши на липшицевых кривых. Позднее появились другие доказательства этой теоремы (см., например, [34]). Чтобы сразу пояснить ее важность, отметим, что оператор $B_{\omega}$ на липшицевой поверхности приходится рассматривать как сингулярньй интегральный оператор даже в случае уравнения Лапласа или Гельмгольца: только на гладких поверхностях это интегральньй оператор со слабой особенностью ядра. В случае же системы Ламе это всегда сингулярньй интегральньй оператор, даже если поверхность Г гладкая. Сужения на Г производных от потенциала простого слоя по касательным направлениям также являются сингулярными интегральньми операторами.

Теорема из [26] содержит существенную дополнительную информацию о поведении потенциалов вблизи липшищевой границы. Чтобы изложить ее, нам понадобится несколько определений.

В начале п. 2.4 мы определили конусы $C^{ \pm}(x), x \in \Gamma$. Если функция $u(y)$ имеет предел $u^{ \pm}(x)$ при $C^{ \pm}(x) \ni y \rightarrow x$ для почти всех $x \in \Gamma$, то будем говорить, что она имеет граничное значение $u^{ \pm}(x)$ в смысле некасательной сходимости. Аналогично граничное значение $\partial_{\nu_{a}} u^{ \pm}$конормальной производной в смысле некасательной сходимости определим как предел величины $\partial_{\nu_{a}(x)} u(y)$ при $C^{ \pm}(x) \ni y \rightarrow x$ для почти всех $x$, если такой предел сушествует.

Для функций $u(x)$ в $\Omega^{ \pm}$определим максимальные функции

$$
u_{*}^{ \pm}(x)=\sup _{y \in C^{ \pm}(x)}|u(y)|
$$

Обозначим через $V_{\omega}\left(\Omega^{+}\right)$класс всех решений $u$ системы $(4.24)$ в $\Omega^{+}$с $u_{*}^{+} \in L_{2}(\Gamma)$ и некасательным граничным значением $u^{+}$. Через $W_{\omega}\left(\Omega^{+}\right)$обозначим подкласс этого класса с $(\operatorname{grad} u)_{*}^{+} \in L_{2}(\Gamma)$ и некасательньм граничньм значением $\operatorname{grad} u^{+}$. Автоматически эти граничные значения принадлежат $L_{2}(\Gamma)$, а у функции $u$ из $W_{\omega}\left(\Omega^{+}\right)$ сушествует некасательное граничное значение конормальной производной, принадлежашее $L_{2}(\Gamma)$. Сp. [120]. Классы $V_{\omega}\left(\Omega^{-}\right)$и $W_{\omega}\left(\Omega^{-}\right)$определяются аналогично, но дополнительно решения подчиняются нужным условиям на бесконечности.

Мы не будем приводить несколько громоздкую формулировку общего результата из упомянутой вьше работы [26] и сразу укажем следствия для операторов $A_{\omega}, \mathscr{A}_{\omega}$, $B_{\omega}$ и $\mathscr{B}_{\omega}$ (см., например, [123] и [40]). 
Tеорема 5.3. Оператор $A_{\omega}$ действует ограниченным образом из $H^{0}(\Gamma)$ в $H^{1}(\Gamma)$, а значения потенциала $\mathscr{A}_{\omega} \varphi$ при $\varphi \in H^{0}(\Gamma)$ принадлежат $W_{\omega}\left(\Omega^{ \pm}\right)$. Оператор $B_{\omega}$ действует ограниченным образом в $H^{0}(\Gamma)$, а значения потенциала $\mathscr{B}_{\omega} \psi$ при $\psi \in H^{0}(\Gamma)$ принадлежсат $V_{\omega}\left(\Omega^{ \pm}\right)$. При этом

$$
\left\|\left(\operatorname{grad} \mathscr{A}_{\omega} \varphi\right)_{*}^{ \pm}\right\|_{0, \Gamma}+\left\|\left(\mathscr{A}_{\omega} \varphi\right)_{*}^{ \pm}\right\|_{0, \Gamma} \leqslant C\|\varphi\|_{0, \Gamma} \quad u \quad\left\|\left(\mathscr{B}_{\omega} \psi\right)_{*}^{ \pm}\right\|_{0, \Gamma} \leqslant C\|\psi\|_{0, \Omega}
$$

Теперь мы сформулируем теорему о связи квадратичной интегрируемости максимальных функщий решений с принадлежностью этих решений к обычным пространствам Соболева.

ТеОРема 5.4. Решение и сильно әллиптической системы (4.24) принадлежит $H^{1 / 2}\left(\Omega^{+}\right)$в том и только том случае, если $u_{*}^{+} \in L_{2}(\Gamma)$, и принадлежст $H^{3 / 2}\left(\Omega^{+}\right)$в том и только том случае, если $u_{*}^{+} \in L_{2}(\Gamma) u(\operatorname{grad} u)_{*}^{+} \in L_{2}(\Gamma)$. Аналогично, решение и системы (4.24) в $\Omega^{-}$принадлежит $H_{\mathrm{loc}}^{1 / 2}\left(\Omega^{-}\right)$в том и только том случае, если $u_{*}^{-} \in L_{2}(\Gamma)$, и принадлежит $H_{\mathrm{loc}}^{3 / 2}\left(\Omega^{-}\right)$в том и только том случае, если $u_{*}^{-} \in L_{2}(\Gamma) u(\operatorname{grad} u)_{*}^{-} \in L_{2}(\Gamma)$. При $\omega^{\prime \prime}>0$ индекс lос можно onycmumb.

Для уравнений Гельмгольца и Ламе это утверждение фактически доказано в [15] и [12], но там формулировки содержат дефект. В [9; $\S 6]$ теорема 5.4 в приведенной здесь формулировке получена для общих систем (4.24), а в [92] - для систем в $\Omega$ с переменными коэффициентами. Упомянем, что это утверждение доказьвается в [9] сначала в $\Omega$ при $\omega=0$ с использованием результатов из [33] и [53]. При этом выводятся оценки норм $\left\|u_{*}^{+}\right\|_{0, Г}$ и $\|u\|_{1 / 2, \Omega}$ через норму $\left\|\rho^{1 / 2}(x) \operatorname{grad} u(x)\right\|_{0, \Omega^{+}}$и обратные оценки (с добавлением младших членов). Здесь $\rho(x)$ - расстояние от точки $x \in \Omega^{+}$до границы.

В качестве следствия получаем, что в определении классов $V_{\omega}\left(\Omega^{ \pm}\right)$условия $u_{*}^{+} \in L_{2}(\Gamma)$ и $u_{*}^{-} \in L_{2}(\Gamma)$ эквивалентны соответственно условиям $u \in H^{1 / 2}\left(\Omega^{+}\right)$и $u \in H_{\mathrm{loc}}^{1 / 2}\left(\Omega^{-}\right)$. Аналогично в определении классов $W_{\omega}\left(\Omega^{ \pm}\right)$условия $u_{*}^{+} \in L_{2}(\Gamma)$, $\operatorname{grad} u_{*}^{+} \in L_{2}(\Gamma)$ и условия $u_{*}^{-} \in L_{2}(\Gamma), \operatorname{grad} u_{*}^{-} \in L_{2}(\Gamma)$ эквивалентны соответственно условию $u \in H^{3 / 2}\left(\Omega^{+}\right)$и условию $u \in H_{\mathrm{loc}}^{3 / 2}\left(\Omega^{-}\right)$.

Для оператора $\mathscr{A}_{\omega}$ получается, что он действует ограниченным образом из $H^{0}(\Gamma)$ в $H^{3 / 2}\left(\Omega^{+}\right)$и $H_{\mathrm{loc}}^{3 / 2}\left(\Omega^{-}\right)$, а оператор $\mathscr{B}_{\omega}-$ из $H^{0}(\Gamma)$ в $H^{1 / 2}\left(\Omega^{+}\right)$и в $H_{\mathrm{loc}}^{1 / 2}\left(\Omega^{-}\right)$. При этом функция $\mathscr{A}_{\omega} \varphi$ с $\varphi \in H^{0}(\Gamma)$ и ее градиент имеют некасательные граничные значения соответственно в $H^{1}(\Gamma)$ и $H^{0}(\Gamma)$, и функция $\mathscr{B}_{\omega} \psi$ с $\psi \in H^{0}(\Gamma)$ имеет некасательные граничные значения в $H^{0}(\Gamma)$.

Известно, что связная липшицева поверхность Г допускает аппроксимацию бесконечно гладкими замкнутьми связными поверхностями $\Gamma_{j}=\Gamma_{j}^{ \pm}$, лежашими соответственно в $\Omega^{ \pm}$и связанньми с Г липшицевым диффеоморфизмом $\Lambda_{j}^{ \pm}: \Gamma \rightarrow \Gamma_{j}^{ \pm}$. Липшицевы постоянные этих диффеоморфизмов и обратных к ним отображений ограничены равномерно по $j$. Точки $\Lambda_{j}^{ \pm}(x)$ равномерно сходятся к $x \in \Gamma$, оставаясь внутри конусов $C^{ \pm}(x)$. Единичные векторы внешних нормалей $\nu\left(\Lambda_{j}^{ \pm}(x)\right)$ к $\Gamma_{j}^{ \pm}$сходятся к $\nu(x)$ почти всюду и в $L_{2}(\Gamma)$. Интегралы от функций по $\Gamma_{j}^{ \pm}$преобразуются в интегралы по $\Gamma$ с дополнительным множителем (модулем якобиана), которьй заключен между положительными постоянными и стремится к 1 почти всюду и в $L_{2}(\Gamma)$. Этот результат 
принадлежит Нечасу [100]; удобную формулировку и доказательство см., например, в [31].

Используя эту аппроксимацию, можно проверить

ПреДЛОЖЕНИЕ 5.5. Формульи (4.30) и первые формульи в (4.31) остаются справедливьми для $\varphi$ и $\psi$ из $H^{0}(\Gamma)$.

Формула Грина (4.37) справедлива для решений $u, v$ из $W_{\omega}\left(\Omega^{+}\right)$. Аналогичная формула Грина справедлива в $\Omega_{R}^{-}$.

Для решений из $W_{\omega}\left(\Omega^{ \pm}\right)$справедливь интегральные формуль (4.33) и соотношения на граниче (4.34).

Далее, в случае липшицевой $Г$ при всех допустимых $\omega$ сохраняется единственность для внешних задач Дирихле и Неймана в $W_{\omega}^{-}$, она проверяется так же, как в случае гладкой гранищы.

ПРЕДЛОЖЕНИЕ 5.6. Формуль (4.35) для $\operatorname{Ker} A_{\omega} u \operatorname{Ker}\left(B_{\omega}^{\prime}+\frac{1}{2} I\right)$ верны в $H^{0}(\Gamma)$ с $u \in W_{\omega}\left(\Omega^{+}\right)$. Для ядра $\operatorname{Ker}\left(B_{\omega}-\frac{1}{2} I\right)$ в $H^{1}(\Gamma)$ справедлива формула (4.36). Формула (4.45) сохраняется на функциях из $H^{0}(\Gamma)$.

Доказательства см. в [9].

Далее встает вопрос об обратимости операторов $A_{\omega}$ и $\frac{1}{2} I \pm B_{\omega}$. Вместо вариационного подхода этот вопрос теперь в ряде работ исследован при помоши так называемых тождеств Реллиха, из которых вьводятся неравенства Реллиха. Они появились в литературе уже давно, см. [106] и [101]. В применении к системам (4.24) неравенства Реллиха в идеальной форме имеют вид

$$
\left\|\partial_{\nu_{a}} u^{ \pm}\right\|_{0, \Gamma} \leqslant C_{1}\left\|\operatorname{grad}_{\tau} u^{ \pm}\right\|_{0, \Gamma} \quad \text { и }\left\|\operatorname{grad}_{\tau} u^{ \pm}\right\|_{0, \Gamma} \leqslant C_{2}\left\|\partial_{\nu_{a}} u^{ \pm}\right\|_{0, \Gamma}
$$

для решений однородной системы (4.24) в $\Omega^{ \pm}$, где $\operatorname{grad}_{\tau}$ - тангенциальная составляюшая градиента. Фактически в правых частях этих неравенств обычно присутствуют некоторые младшие члены. Эти неравенства удивительным образом позволяют обойтись без понятия эллиптичности и доказать обратимость или хотя бы фредгольмовость для $A_{\omega}$ как оператора из $H^{0}(\Gamma)$ в $H^{1}(\Omega)$ и для $\frac{1}{2} I \pm B_{\omega}$ и $\frac{1}{2} I \pm B_{\omega}^{\prime}$ как операторов в $H^{0}(\Gamma)$. Мы не будем объяснять, как используются неравенства Реллиха, с этим можно познакомиться, например, по работе [123] о задачах Дирихле и Неймана для уравнения Лапласа и работе [32] по этим же задачам для системы Ламе, см. также уже упомянутые обзоры [52], [59] и [60].

Однако приведем следующее пояснение. Неравенства (5.6) по существу являются априорньми оценками для операторов $\mathscr{T}_{\omega}^{ \pm}$из $H^{1}(\Gamma)$ в $H^{0}(\Gamma)$ и обратных операторов $T_{\omega}^{ \pm}$.

Сейчас мы посмотрим, как складьвается картина, если уже известно, что $A_{\omega}$ обратим как оператор из $H^{0}(\Omega)$ в $H^{1}(\Omega)$ и $B_{\omega}^{\prime} \pm \frac{1}{2} I$ обратимы как операторы в $H^{0}(\Omega)$. В частности, $\omega$ сейчас предполагается не исключительным относительно внутренних задач Дирихле и Неймана. Если мы располагаем всеми неравенствами Реллиха, то обратимость этих операторов доказьвается одновременно.

Из формулы (4.45) следует, что оператор $\mathscr{B}_{\omega}=A_{\omega} B_{\omega}^{\prime} A_{\omega}^{-1}$ ограничен в $H^{1}(\Gamma)$. Из соображений интерполящии получается, что $B_{\omega}$ действует ограниченньм образом в 
$H^{t}(\Gamma), 0 \leqslant t \leqslant 1$, причем это верно для всех рассматриваемых сейчас $\omega$. В свою очередь, отсюда следует, что транспонированный оператор $B_{\omega}^{\prime}$ продолжается до ограниченного оператора в $H^{-t}(\Gamma)$ при тех же $t$, или, что то же, в $H^{t-1}$ при тех же $t$.

Далее, из обратимости $A_{\omega}$ как оператора из $H^{0}(\Gamma)$ в $H^{1}(\Gamma)$ ввиду его симметрии следует его обратимость из $H^{-1}(\Gamma)$ в $H^{0}(\Gamma)$. Используя интерполяцию, получаем, что он обратим как оператор из $H^{t-1}(\Gamma)$ в $H^{t}(\Gamma)$ при $0 \leqslant t \leqslant 1$.

Кроме того, получается обратимость оператора $B_{\omega} \pm \frac{1}{2} I$ в $H^{1}(\Gamma)$ :

$$
\left(B_{\omega} \pm \frac{1}{2} I\right)^{-1}=A_{\omega}\left(B_{\omega}^{\prime} \pm \frac{1}{2} I\right)^{-1} A_{\omega}^{-1}
$$

Как следствие этот оператор обратим в $H^{t}(\Gamma)$ при $0 \leqslant t \leqslant 1$.

Для решения задачи $D_{\omega}^{+}$мы теперь имеем две знакомые нам формулы

$$
u=\mathscr{A}_{\omega} A_{\omega}^{-1} u^{+} \text {и } u=\mathscr{B}_{\omega}\left(B_{\omega}+\frac{1}{2} I\right)^{-1} u^{+} .
$$

Можно считать, что в первой из этих формул $u^{+} \in H^{1}(\Gamma)$, а во второй $u^{+} \in H^{0}(\Gamma)$. Но при $u^{+} \in H^{1}(\Gamma)$ мы имеем $\left(B_{\omega} \pm \frac{1}{2} I\right)^{-1} u^{+} \in H^{1}(\Gamma)$; с учетом единственности для задачи Дирихле получается, что сужение оператора $\mathscr{B} \omega$ на $H^{1}(\Gamma)$ действует ограниченным образом из этого пространства в $H^{3 / 2}(\Omega)$. Далее, $A^{-1} u^{+} \in H^{-1}(\Gamma)$ при $u^{+} \in H^{0}(\Gamma)$. Отсюда получается, что $\mathscr{A}_{\omega}$ продолжается до ограниченного оператора из $H^{-1}(\Gamma)$ в $H^{1 / 2}(\Omega)$. Немного подробнее: если $\varphi \in H^{-1}(\Gamma)$ и $A_{\omega} \varphi=\psi$, то $\psi \in H^{0}(\Gamma)$. Пусть $\left\{\psi_{n}\right\}$ - последовательность функций из $H^{1}(\Gamma)$, сходящаяся к $\psi$ в $H^{0}(\Gamma)$. Положим $u_{n}^{+}=\psi_{n}$; тогда решения

$$
u_{n}=\mathscr{A}_{\omega} A_{\omega}^{-1} u_{n}^{+}=\mathscr{B}_{\omega}\left(B_{\omega}+\frac{1}{2} I\right)^{-1} u_{n}^{+}
$$

задачи Дирихле принадлежат $H^{3 / 2}(\Omega)$ и сходятся к некоторой функции $u$ в $H^{1 / 2}(\Omega)$. Полагаем $u=\mathscr{A}_{\omega}^{+} \varphi . \mathrm{Cp} .[28]$ и [88].

С учетом интерполящии в итоге получается, что задача Дирихле $D_{\omega}^{+}$для однородной системы (4.24) имеет решение в $H^{t+1 / 2}(\Omega)$ при $u^{+} \in H^{t}(\Gamma), 0 \leqslant t \leqslant 1$, которое строится по любой из формул (5.7). Концы этого промежутка не были достижимы, пока мы пользовались только вариационным методом. Аналогично строится решение задачи $D_{\omega}^{-}$в $H_{\mathrm{loc}}^{t+1 / 2}(\Omega)$ при $u^{-} \in H^{t}(\Gamma)$ для тех же $t$.

Решение задачи $N_{\omega}^{+}$в $H^{3 / 2}(\Omega)$ при $\partial_{\nu_{a}} u^{+} \in H^{0}(\Gamma)$ строится по формуле

$$
u=\mathscr{A}_{\omega}\left(B_{\omega}^{\prime}-\frac{1}{2} I\right)^{-1} \partial_{\nu_{a}} u^{+}
$$

Но эта формула сохраняет смысл при $\partial_{\nu_{a}} u^{+} \in H^{-1}(\Gamma)$. Можно показать, что она согласуется с прежним определением решения задачи Неймана для однородной системы. С учетом интерполяции заключаем, что задача $N_{\omega}^{+}$имеет решение в $H^{t+1 / 2}(\Omega)$ nри $\partial_{\nu_{a}} u^{+} \in H^{t-1}(\Gamma)$, если $0 \leqslant t \leqslant 1$. Снова удалось присоединить конщы к интервалу $(0,1)$, что было недостижимо, пока мы пользовались только вариационньм методом. Для внешней задачи Неймана получаются аналогичные результаты: разрешимость в $H_{\mathrm{loc}}^{t+1 / 2}(\Omega)$ при $\partial_{\nu_{a}} u^{-} \in H^{t-1}(\Gamma)$ для тех же $t$. 
Для операторов $T_{\omega}^{ \pm}$мы теперь получаем формулы

$$
T_{\omega}^{ \pm}=A_{\omega}\left(B^{\prime} \mp \frac{1}{2} I\right)^{-1}
$$

и видим, что это ограниченные обратимые операторы из $H^{t-1}(\Gamma)$ в $H^{t}(\Gamma)$ при $0 \leqslant t \leqslant 1$. Ранее вместо этого отрезка мы имели только интервал $(0,1)$.

K сожалению, вторые неравенства Реллиха на обших липшищевых поверхностях в пределах класса систем вида (4.24), по-видимому, известны только при условии III, т.е. для уравнения Гельмгольца, а также для системы Ламе, причем для системы Ламе их проверка в [32] является достаточно сложной. Только в этих случаях мы пока можем рассматривать операторы $T_{\omega}^{ \pm}$в только что указанных пространствах при $0 \leqslant t \leqslant 1$. Уже для системы анизотропной упругости мы, вообще говоря, не имеем вторых неравенств Реллиха и можем пользоваться только вариационньм подходом.

Однако первые неравенства Реллиха известны в общем случае сильно эллиптических систем. В работе Гао [40] при $n \geqslant 3$ на этой основе исследованы задача Дирихле для общей сильно эллиптической системы без младших членов и соответствующий оператор $A_{0}$. При этом вместо задачи Неймана, следуя работе Кальдерона [25], Гао рассматривает более легкую задачу с косой производной вдоль нетангенциального векторного поля, удовлетворяющего на поверхности условию Липшица. Двумерншй случай для системы с переменными коэффициентами исследован в [87].

ЗАмЕчАниЕ 5.7. Вернемся к теореме 5.4 и приведем пример, показывающий, что утверждение теоремы 3.2 теряет силу для $t=1 / 2$ [53]. Пусть $v$-такая функция из $H^{3 / 2}(\Omega)$, что ее граничное значение не принадлежит $H^{1}(\Gamma)$. Допустим, что решение задачи Дирихле $\Delta u=\Delta v$ в $\Omega, u^{+}=0$ принадлежит $H^{3 / 2}(\Omega)$. Тогда $w=u-v-$ решение уравнения Лапласа из $H^{3 / 2}(\Omega)$. В силу теоремы 5.4 его граничное значение принадлежит $H^{1}(\Gamma)$. Получается противоречие с тем, что $v^{+}$не принадлежит $H^{1}(\Gamma)$.

Что касается значения $t=-1 / 2$, то для него теорема 3.2 неверна даже в случае области с гладкой границей. Действительно, пусть $v$ - функция из $H^{1 / 2}(\Omega)$ с граничным значением, не принадлежашим $H^{0}(\Omega)$. Допустим, что решение задачи Дирихле $\Delta u=\Delta v$ в $\Omega, u^{+}=0$ принадлежит $H^{1 / 2}(\Omega)$. Тогда $w=u-v$-решение уравнения Лапласа из $H^{1 / 2}(\Omega)$, и по теореме 5.4 его граничное значение принадлежит $H^{0}(\Gamma)$. Снова получается противоречие.

Теперь мы приведем теорему о спектральных свойствах оператора $A_{\omega}$, несколько усиливающую соответствующий результат из [9]. Предположим вьполненными условие I сильной эллиптичности и, если $\omega>0$, условие V. Определение почти гладкой поверхности, используемое в формулировке, приведено в конце п. 1 введения.

Теорема 5.8. Оператор $A_{\omega}$ комплексно симметричен при всех допустимых $\omega$ и является самосопряженным при $\omega^{\prime}=0$. При остальных $\omega$ это слабое возмущение оператора $A_{0}$.

Пусть задача $D_{\omega}^{+}$однозначно разрешима. Тогда $A_{\omega}$ - обратимый оператор из $H^{t-1}(\Gamma)$ в $H^{t}(\Gamma)$ nри $0 \leqslant t \leqslant 1$.

Если $A_{\omega}=A_{\omega}^{*}$, то собственные функции этого оператора принадлежсат $H^{1}(\Gamma)$ и составленный из них ортонормированный базис в $H^{0}(\Gamma)$ остается безусловным базисом в $H^{t}(\Gamma)$ при $|t| \leqslant 1$.

Если $A_{\omega} \neq A_{\omega}^{*}$, то корневье функиии оператора $A_{\omega}$ принадлежсат $H^{1}(\Gamma)$. При $n \leqslant 3$ из них можсно составить безусловньй базис со скобками сразу во всех 
$H^{t}(\Gamma),|t| \leqslant 1$, а при $n>3-$ базис для метода суммирования Абеля-Лидского любого порядка больие $n-3$ в этих пространствах.

Действительные части $\operatorname{Re} \mu_{j}$ характеристических чисел стремятся $\kappa-\infty$. При всех допустимых $\omega$ справедлива оценка $0<C_{1} \leqslant\left|\mu_{j}\right| / j^{1 /(n-1)}$. Eсли $\omega^{\prime}=0$, то справедлива двусторонняя оценка $0<C_{1} \leqslant\left|\mu_{j}\right| / j^{1 /(n-1)} \leqslant C_{2}$. Если Гпочти гладкая поверхность, то

$$
\mu_{j}=-C_{n} j^{1 /(n-1)}+o\left(j^{1 /(n-1)}\right),
$$

$C_{n}>0$, при всех допустимых $\omega$.

Eсли $\omega^{\prime} \neq 0$, mo $\operatorname{Im} \mu_{j}=O\left(j^{-h}\right)$, где $h=1 /(n-1)$. Onepamop $\pm A_{\omega}$ duccunamuвен при $\mp \omega^{\prime}>0$, так что знаки этих мнимых частей совпадают со знаком $\omega^{\prime}$.

Все действительные части $\operatorname{Re} \mu_{j}$ отрицательны при $\left|\omega^{\prime}\right| \leqslant \omega^{\prime \prime}$.

ДокАЗАТЕльство в основном аналогично доказательству теоремы 4.13 , но в случаях, когда оператор $A_{\omega}$ несамосопряженный, мы рассматриваем его как слабое возмушение, скажем, оператора $A_{i}$, а не $\operatorname{Re} A_{\omega}$. Особенность ядра разности $A_{\omega}-A_{i}$ на два порядка ниже, чем у ядра оператора $A_{i}$. Чтобы полностью использовать это обстоятельство, мы рассматриваем $A_{i}$ и $A_{\omega}$ как операторы в $H^{-1}(\Gamma)$ со скалярным произведением, в котором $A_{i}$ - самосопряженный оператор.

Асимптотика для $\mu_{j}$ в случае почти гладкой Г следует из результата в [11], если $\omega^{\prime}=0$, и переносится на остальные $\omega$ при помоши теоремы Келдьша (см. предложение 2.15).

Отметим, что в [15] указан пример собственной функции оператора $A_{\omega}$ для уравнения Гельмгольца, отвечающей нулевому собственному значению и не принадлежащей $H^{1}(\Gamma)$. В подобных случаях линейная замкнутая оболочка корневых функций в $H^{1}(\Gamma)$ имеет конечномерньй дефект.

Используя обратимость операторов $B_{\omega} \pm \frac{1}{2} I$, мы можем образовать операторы $T_{\omega}^{ \pm}$ и $T_{\omega}$ по формулам $T_{\omega}^{ \pm}=\left(B_{\omega} \mp \frac{1}{2} I\right)^{-1} A_{\omega}$ и $T_{\omega}=T_{\omega}^{+}+T_{\omega}^{-}$. Если мы располагаем вторьми неравенствами Реллиха, то эти операторы действуют из $H^{0}(\Omega)$ в $H^{1}(\Omega)$ и для них получаются такие же спектральные результаты, как для оператора $A_{\omega}$, включая асимптотическую формулу (5.10) для характеристических чисел в случае почти гладкой поверхности. См. [11].

Сушественная разница состоит только в том, что оператор $T_{\omega}^{+}$является самосопряженным не только при чисто мнимых, но и при положительных $\omega$.

Если же вторых неравенств Реллиха нет, то мы должны рассматривать эти операторы как действуюшие из $H^{t-1}(\Gamma)$ в $H^{t}(\Gamma)$ при $0<t<1$. Но формула $(5.10)$ для характеристических чисел в случае почти гладкой поверхности сохраняется.

5.3. Системы с переменными коэффициентами и метод потенциалов. В работе [91], см. также [89], теорема из [26] распространена на случай потенщиалов для систем с переменными коэффициентами. Затем исследованы задача Дирихле для обших сильно эллиптических систем в области $\Omega$ на замкнутом многообразии и оператор $A$ типа потенциала простого слоя. В этой работе предполагается, что $n \geqslant 3$, но двумерный случай охвачен в работе [87]. Кроме того, для скалярных уравнений типа 
Шрёдингера в [91] исследована задача Неймана, а в [89] изучена серия "естественных задач" для лапласиана Ходжа на формах в липшицевой подобласти риманова многообразия. Относительно более ранних результатов по задаче Дирихле см. [51], [61] и приведенные там ссылки.

В отношении спектральных свойств интересуюших нас операторов это создает возможности, объясненные в предыдущем пункте в случае системы с постоянными коэффициентами. А именно, в случае общих сильно эллиптических систем можно рассматривать $A_{\omega}$ как самосопряженньй оператор или слабое возмущение самосопряженного оператора в $H^{0}(\Gamma)$ с областью значений $H^{1}(\Gamma)$, а также как оператор в $H^{-1}(\Gamma)$ с областью значений $H^{0}(\Gamma)$. В частности, в случае формальной самосопряженности системы и $\omega^{\prime}=0$ получается, что собственные функции этого оператора образуют безусловный базис в $H^{t}(\Gamma)$ при $|t| \leqslant 1$ - это наилучший возможньй результат, в п. 5.1 концы этого отрезка не были доступны. В скалярном случае то же самое получается для операторов $T_{\omega}^{ \pm}$и $T_{\omega}$. При этом явные формулы для этих операторов позволяют получить асимптотические формулы для их характеристических чисел, если поверхность Г почти гладкая и коэффициенты системы бесконечно гладкие (или достаточно гладкие) в силу результатов из [11].

Не недоступен и случай комплексных старших коэффициентов в системе с эрмитовым главным символом.

\section{§ 6. Добавление к главе II. Некоторые спектральные задачи для систем Максвелла и Дирака}

В этом добавлении мы кратко рассмотрим спектральные задачи со спектральным параметром на границе для классических систем первого порядка - Максвелла и Дирака. Эти задачи сводятся к спектральным уравнениям с неэллиптическими операторами на $\Gamma$, спектральные свойства которых оказываются несколько необычными. Эти неэллиптические уравнения удается в результате некоторого трюка преобразовать в эллиптические, что и приводит к искомьм результатам. Задачи для обеих систем исследуются похожим образом.

6.1. Спектральные задачи для системы Максвелла. Система Максвелла имеет вид

$$
\operatorname{rot} \mathbf{H}-i k \mathbf{E}=0, \quad \operatorname{rot} \mathbf{E}+i k \mathbf{H}=0 .
$$

Здесь $\mathbf{E}$ и $\mathbf{H}$ - трехмерные векторные поля (электрическая и магнитная напряженности). Мы рассмотрим здесь одну из спектральных задач, поставленных в [126]. Подробности можно найти в [1] или в [14; гл. 5]. Эта задача состоит в нахождении решений системы (6.1) в ограниченной области $\Omega \subset \mathbb{R}^{3}$ с граничньм условием

$$
\mu\left(\nu \times \mathbf{H}^{+}\right)-i \mathbf{E}_{\tau}^{+}=0
$$

где $\times$ - векторное произведение и $\mathbf{E}_{\tau}^{+}$- касательная составляющая поля $\mathbf{E}$ на границе; $\mu$-спектральньй параметр. Граница Г предполагается бесконечно гладкой и связной. Для экономии места мы будем считать $k$ положительньм и отличньм от (счетного набора) значений $k=k_{j}$, при которых система (6.1) в $\Omega$ с граничным условием $\nu \times \mathbf{E}^{+}=0$ имеет нетривиальные решения. 
Используя фундаментальное решение для уравнения Гельмгольца, можно написать интегральное представление полей $\mathbf{E}$ и $\mathbf{H}$ в $\Omega$ через поля $\varphi=-\nu \times \mathbf{H}^{+}$и $\psi=\nu \times \mathbf{E}^{+}$, касательные к Г. (Cp. [27].) Переходя в этом представлении на границу, получаем два соотношения на ней, которые мы формально запишем, сохраняя обозначения из [1] и главы 5 в [14], в виде

$$
(I-B) \psi=i A \varphi, \quad(I-B) \varphi=-i A \psi
$$

Здесь $A$ и $B$ - некоторые $(2 \times 2)$-матричные ПДО на Г порядков 1 и -1 . При наложенном вьше условии на $k$ оператор $I-B$ оказьвается обратимым. Из (6.3) вытекает несколько странное, на первьй взгляд, соотношение

$$
\left[(I-B)^{-1} A\right]^{2}=I
$$

на бесконечно гладких касательных к Г векторных полях. Из него, в частности, следует, что главньй символ ПДО $(I-B)^{-1} A$ вырожден, а квадрат этого главного символа тождественно равен нулю. Это подтверждается прямым вычислением.

Поля $\mathbf{E}$ и Н удовлетворяют векторному уравнению Гельмгольца (так что мы не так уж далеко ушли от сильно эллиптических систем второго порядка), поэтому естественно спектральную задачу рассматривать в классе бесконечно гладких в $\bar{\Omega}$ решений. В этом классе она эквивалентным образом сводится к уравнению в классе бесконечно гладких на Г векторных полей

$$
\mathscr{T} \varphi=\mu \varphi, \quad \text { где } \quad \mathscr{T}=-\nu \times(I-B)^{-1} A .
$$

Этот ПДО первого порядка не эллиптичен. Но из (6.4) видно, что в классе бесконечно гладких векторных полей он имеет обратный $\mathscr{T}^{-1}=(I-B)^{-1} A \nu \times$, тоже ПДО первого порядка. ПДО $\mathscr{T}^{-1}$ тоже не эллиптичен. Но вычисление главного символа ПДО $\Theta=\mathscr{T}-\mathscr{T}^{-1}$ показьвает, что этот ПДО первого порядка эллиптичен. Этот трюк был найден в [13]. В системе координат, использованной в п. 4.3, главный символ ПДО $\Theta$ равен $k^{-1}|\xi| I$, где $I$ - единичная матрица.

Оператор $\mathscr{T}$ является формально самосопряженным, а оператор $\Theta$ - самосопряженным оператором с дискретным спектром (и областью определения $H^{1}(\Omega)$ ), его собственные значения $\mu_{j}(\Theta)$, занумерованные в порядке неубывания с учетом кратностей, имеют асимптотику $c j^{1 / 2}+O(1)$ с положительной постоянной $c$. Любое собственное подпространство оператора $\Theta$, отвечающее фиксированному собственному значению $\mu(\Theta)$, инвариантно относительно $\mathscr{T}$, так что собственные значения оператора $\mathscr{T}$ в этом подпространстве являются корнями квадратного уравнения $\mu-\mu^{-1}=\mu(\Theta)$, притом, как можно показать, это собственные значения одинаковой кратности, если они различны.

Отсюда следует, что в $L^{2}(\Gamma)$ имеется ортонормированный базис из собственных функиий оператора $\mathscr{T}$, остающийся безусловным базисом во всех соболевских пространствах $H^{t}(\Gamma)$. Соответствующие собственные значения образуют две серии с пределами $+\infty$ и 0 . Можно написать асимптотические формулы для этих серий.

Можно еше рассмотреть внешнюю задачу и задачу сопряжения со спектральным параметром в условиях на Г. См. [126] и [1] или [14]. 
6.2. Спектральные задачи для системы Дирака. Рассмотрим задачу для стационарной системы Дирака в трехмерной ограниченной области $\Omega$; эта система описьвает движение свободной частищы. Эту $(2 \times 2)$-систему можно записать в виде

$$
a(D) u(x)-b_{1} v(x)=0, \quad a(D) v(x)-b_{2} u(x)=0, \quad \text { где } a(D)=\sum \sigma_{j} D_{j},
$$

здесь $D=\left(D_{1}, D_{2}, D_{3}\right), D_{j}=-i \partial_{j}$ и $\sigma_{j}$ - три $(2 \times 2)$-матрицы Паули, удовлетворяюшие соотношениям $\sigma_{j}^{2}=I$ и $\sigma_{j} \sigma_{k}=-\sigma_{k} \sigma_{j}$ при $j \neq k$. Граничное условие имеет вид (cp. [119])

$$
i a(\nu) v^{+}=\mu u^{+}
$$

где $\mu$ - спектральный параметр. Границу считаем бесконечно гладкой и связной. Квадрат матрицы $a(\nu)$ равен единичной матрище $I$. Числа $b_{1}$ и $b_{2}$ предположим вешественными и отличньми от нуля и положим $k_{2}=b_{1} b_{2}$. Все дальнейшие преобразования проводятся в классе решений, бесконечно гладких в $\bar{\Omega}$. Подробности см. в [10].

Удобно применением оператора $a(D)$ к уравнениям в (6.6) преобразовать эту систему в эквивалентные ей системы

$$
\begin{aligned}
\Delta u(x)+k^{2} u(x)=0, & a(D) u(x)-b_{1} v(x)=0, \\
\Delta v(x)+k^{2} v(x)=0, & a(D) v(x)-b_{2} u(x)=0 .
\end{aligned}
$$

Опять возникло векторное уравнение Гельмгольца. Предположим $k^{2}$ таким, что внутренняя однородная задача Дирихле для этого уравнения не имеет нетривиальных решений (этим исключаются некоторые положительные $k^{2}=k_{j}^{2} \rightarrow \infty$ ). Теперь используем элементарные результаты из п. 4.1. Если $\mathscr{A}=\mathscr{A}_{k}-$ соответствующий потенщиал простого слоя и $A=A_{k}$ - его сужение на $\Gamma$, то $u=\mathscr{A} A^{-1} u^{+}, v=b_{1}^{-1} a(D) u$. Положим

$$
M \varphi=i\left[a(D) \mathscr{A} A^{-1} \varphi\right]^{+} .
$$

Несложное вычисление показывает, что это неэллиптический ПДО 1-го порядка и $M^{2}=-k^{2} I$. Последнее получается в силу возможности поменять ролями буквы $u$ и $v$. Спектральная задача эквивалентным образом преобразуется в уравнение на $\Gamma$ :

$$
\mathscr{T} \varphi=\mu \varphi, \quad \text { где } \quad \mathscr{T}=b_{1}^{-1} a(\nu) M, \quad \varphi=u^{+} .
$$

Оператор $\mathscr{T}$ - ПДО первого порядка, он не эллиптичен, но имеет на бесконечно гладких функциях обратньй $\mathscr{T}^{-1}=-b_{2}^{-1} M a(\nu)$, тоже неэллиптический ПДО первого порядка. А ПДО $\Theta=b_{1} \mathscr{T}-b_{2} \mathscr{T}^{-1}=a(\nu) M+M a(\nu)$ оказьвается эллиптическим ПДО 1-го порядка с положительным главным символом.

Оператор $\mathscr{T}$ является формально самосопряженным, а оператор $\Theta$ - самосопряженным оператором с дискретным спектром. Любое собственное подпространство оператора $\Theta$ инвариантно относительно $\mathscr{T}$, и собственные значения оператора $\mathscr{T}$ в этом подпространстве - корни квадратного уравнения $b_{1} \mu-b_{2} \mu^{-1}=\mu(\Theta)$, где $\mu(\Theta)-$ соответствуюшее собственное значение оператора $\Theta$. При этом если корни разные, то соответствуюшие собственные значения оператора $\mathscr{T}$ имеют одинаковые кратности. 
Поэтому так же, как в предыдушем пункте, получается, что в $L^{2}(\Gamma)$ имеется ортонормированный базис из собственных функиий оператора $\mathscr{T}$, остающийся безусловныцм базисом во всех соболевских пространствах $H^{t}(\Gamma)$. Соответствующие собственнье значения образуют две серии с пределами $+\infty$ и 0 . Можно написать асимптотические формулы для этих серий.

В [10] рассмотрен также случай, когда одна из постоянных $b_{j}$ равна 0 . Тогда серия стремяшихся к 0 собственных значений заменяется собственньм значением 0 бесконечной кратности. Рассмотрены, кроме того, аналог системы Дирака с переменными бесконечно гладкими коэффициентами (для частицы в электромагнитном поле), некоторые многомерные обобшения, а также внешняя задача и задача сопряжения со спектральным параметром в условиях на $\Gamma$.

\section{§. Заключительные замечания}

7.1. Некоторые итоги. Здесь мы отметим те из продвижений в настоящей статье, которые представляются нам наиболее существенными.

В $\S 2$ мы довели обобщение результатов Саваре для систем с комплексньми старшими коэффициентами до той общности, в которой они получены в [111] для скалярного уравнения с вешественными коэффищиентами. А именно, мы рассмотрели системы с переменными комплексными старшими коэффициентами в шкале пространств $H^{t}(\Omega)$ для решений с $1 / 2<t<3 / 2$. Это обобщение более полно использует те идеи, которые заложены в этой работе. В [9] обобщение было частичным (вещественные постоянные старшие коэффициенты, $1 \leqslant t<3 / 2)$.

В плане построения теории разрешимости задач на основе вариашионного подхода мы определенно следовали работе [28]. Но в то время, когда она была написана, еще не было теоремы о повьшении гладкости решений для систем и не были выяснены рамки доступности второго неравенства Реллиха.

Из спектральных результатов, полученных в настоящей статье, нам кажется наиболее существенным указание соболевских пространств ненулевого порядка в липшицевой области, в которых собственные функции самосопряженной задачи со спектральным параметром в системе образуют безусловный базис. Для задач со спектральным параметром на границе в статье указаны совсем простые соображения, позволившие сделать заключения о спектральных свойствах соответствующих операторов при переменных коэфффициентах и при отсутствии явных представлений для этих операторов.

Мы также выяснили возможности использования двух формул для $R$-матришы, допуская наличие кулоновских особенностей в свободном члене системы.

Сделаем еще следуюшее замечание. Хотя имеется уже много работ по разрешимости эллиптических уравнений и систем второго порядка в негладких областях в различных функциональных пространствах (из которых мы рассматривали только простейшие пространства), нам хотелось бы отметить следуюшее. Шкалы пространств привычны для тех, кто занимается эллиптическими уравнениями в гладких областях, а здесь отчетливая шкала выявилась в результате деятельности ряда авторов для систем 2-го порядка в липшицевых областях. Здесь все важно: и двойственность между пространствами $H^{t}(\Omega)$ и $H_{0}^{-t}(\Omega)$, и совпадение этих пространств при $|t|<1 / 2$, и действие операторов, отвечаюших задачам Дирих ле и Неймана с однородными гра- 
ничными условиями, соответственно из пространств $H^{t}(\Omega)$ и $H_{0}^{t}(\Omega)$ в пространства $H_{0}^{t-2}(\omega)$ и $H^{t-2}(\Omega)$, и интерпретация неоднородных граничных условий Неймана. Автор надеется, что удивительная согласованность всех этих обстоятельств методически достаточно отчетливо продемонстрирована в настоящей статье.

7.2. Открытые вопросы. По мнению автора, следует выделить три таких вопроса.

1. Справедливо ли неравенство типа второго неравенства Реллиха для всех систем второго порядка, для которых коэрцитивна задача Неймана? Пока, по-видимому, положительные результаты известны в основном только для систем, удовлетворяющих условию III (см. [37] и [61]), в частности, для скалярного уравнения Шрёдингера, а также для системы Ламе [32], а отрицательных результатов нет. В тех случаях, когда это неравенство верно, интервал значений $t$, указанньй в начале п. 7.1 , для решений однородной системы с неоднородными граничными условиями Неймана расширяется до отрезка $1 / 2 \leqslant t \leqslant 3 / 2$, как для задачи Дирихле. Было бы интересно понять, какова предельная общность, в которой это верно. Отметим, что некоторые специальные ситуации исследованы в [124]. Кроме того, достижимость точки $3 / 2$ в областях с коническими точками (граница принадлежит $C^{2}$ вне конечного числа таких точек) для систем анизотропной упругости следует из результатов в [66], где использован совершенно иной аппарат. И, как мы уже отмечали, в [89] исследован ряд "естественных задач” для лапласиана Ходжа на формах в липшицевой подобласти риманова многообразия.

Этот вопрос, конечно, не нов для специалистов по задачам в липшицевых областях.

2. Справедлива ли спектральная асимптотика вида (5.10) для самосопряженного оператора $A_{\omega}$ типа потенциала простого слоя (и более обших операторов типа потенциала) на общих липшицевых поверхностях? Имеется обширньй цикл работ Бирмана и Соломяка и их учеников, начиная с Костометова, с исследованием оценок $s$-чисел и спектральных асимптотик для интегральных операторов с негладкими ядрами (см. [20], [65] и приведенные там ссылки). Но уже в случае почти гладких липшицевых поверхностей доказательство формулы (5.10) потребовало новых технических продвижений в [11]. Любопытно отметить, что ожидаемьй коэффициент в не доказанной в случае обшей липшищевой поверхности формуле (5.10), аналогичный известному в случае гладкой поверхности, можно указать, пользуясь тем, что липшищева поверхность дифференцируема почти всюду и поэтому “главный символ” у интересуюших нас операторов можно формально определить для почти всех точек на поверхности.

3. Можно ли перенести спектральные результаты для уравнений Максвелла и Дирака из $§ 6$ со спектральным параметром на Г на случай липшицевых поверхностей? Эти результаты получены там средствами исчисления ПДО, которого нет на липшицевых поверхностях.

7.3. Что не вошло в статью. За рамками этой статьи остались:

спектральные задачи для не однородных относительно дифференцирования систем, эллиптических по Дуглису-Ниренбергу, начиная с системы Стокса;

спектральные задачи, далекие от сопряженных (для систем с неэрмитовьм главным символом);

задачи со спектральным параметром в системе и в граничном условии; 
спектральные задачи для уравнений и систем высших порядков;

спектральные задачи в пространствах, отличных от $H^{t}$;

спектральная функция и суммирование рядов Фурье по собственным функциям методами Рисса;

спектральные задачи со смешанными граничньми условиями.

По этим направлениям имеется немало работ и есть не исследованные вопросы. Но, как справедливо утверждал Козьма Прутков, нельзя объять необъятное.

\section{СПИСОК ЛИТЕРАТУРЫ}

[1] М.С. Агранович. Спектральные свойства задач дифракции // Добавление к книге [126]. С. 288-416.

[2] М.С. Агранович. О сходимости рядов по корневым векторам операторов, очень близких к самосопряженным // Труды ММО. 1980. Т. 41. С. 163-180.

[3] М.С. Агранович. Об эллиптических псевдодифференциальных операторах на замкнутой кривой // Труды ММО. 1984. Т. 47. С. 22-67.

[4] М.С. Агранович. Эллиптические операторы на замкнутых многообразиях // Итоги науки и техники. Совр. проблемы матем. Фунд. направл. Т. 63. Диф. уравнения с частными производньгии VI. М.: ВИНИТИ, 1990. С. 5-129.

[5] М. С. Агранович. О несамосопряженных задачах с параметром, эллиптических по Агмону-Дуглису-Ниренбергу // Функц. анализ и его прил. 1990. Т. 24. № 1. С. 59-61.

[6] М.С. Агранович. О модулях собственных значений несамосопряженных задач с параметром, эллиптических по Агмону-Дуглису-Ниренбергу // Функц. анализ и его прил. 1992. T. 26. № 2. С. 51-55.

[7] М.С. Агранович. О рядах по корневым векторам операторов, определяемых формами с самосопряженной главной частью // Функц. анализ и его прил. 1994. Т. 28. № 3. С. 1-21.

[8] M.S. Agranovich. Elliptic boundary problems // Partial Differential Equations. IX: Elliptic Boundary Value Problems. Berlin: Springer-Verlag, 1997. P. 1-144. (Encyclopaedia Math. Sci. V. 79.)

[9] М. С. Агранович. Спектральные свойства операторов типа потенциала для некоторого класса сильно эллиптических систем на гладких и липшицевых поверхностях // Труды MMO. 2001. T. 62. C. 3-53.

[10] М. С. Агранович. Спектральные задачи для системы Дирака со спектральньм параметром в локалшных граничных условиях // Функц. анализ и его прил. 2001. Т. 35 . № 3. C. $1-18$.

[11] М.С. Агранович, Б. А. Амосов. Оценки $s$-чисел и спектралшные асимптотики для интегралшных операторов типа потенциала на негладкой поверхности // Функц. анализ и его прил. 1996. Т. 30. № 2. С. 1-18.

[12] M.S. Agranovich, B.A. Amosov, M. Levitin. Spectral problems for the Lamé system with spectral parameter in boundary conditions on smooth or nonsmooth boundary // Russian J. Math. Phys. 1999. V. 6. № 3. P. 247-281.

[13] М. С. Агранович, 3. Н. Голубева. О некоторых задачах для системы Максвелла со спектральным параметром в граничном условии // Докл. АН СССР. 1976. Т. 231. № 4. C. $777-780$.

[14] M.S. Agranovich, B.Z. Katsenelenbaum, A. N. Sivov, N. N. Voitovich. Generalized Method of Eigenoscillations in Diffraction Theory. Berlin: Wiley-VCH, 1999.

[15] М.С. Агранович, Р. Менникен. Спектралшные задачи для уравнения Гелшмгольца со спектралшным параметром в граничных условиях на негладкой поверхности // Матем. сб. 1999. Т. 190. № 1. С. 29-68.

[16] М.С. Агранович, М.И. Вишик. Эллиптические задачи с параметром и параболические задачи общего вида // УМН. 1964. Т. 19. № 3. С. 53-161.

[17] 
[18] О.В.Бесов, В. П. Ильин, С. М. Никольский. Интегральные представления функций и теоремы вложения. М.: Наука, 1975.

[19] М.Ш. Бирман, М.З. Соломяк. Спектральная асимптотика негладких эллиптических операторов. I, II // Труды ММО. 1972. Т. 27. С. 3-52; 1973. Т. 28. С. 3-24.

[20] М.Ш. Бирман, М.З. Соломяк. Оценки сингулярных чисел интегральных операторов // УМН. 1977. Т. 32. №1. С. 17-84.

[21] М.Ш. Бирман, М.З. Соломяк. Спектральная теория операторов в гильбертовом пространстве. Л.: Изд-во ЛГУ, 1980.

[22] M. Bramanti. Potential theory for stationary Schrödinger operators: a survey of results obtained with non-probabilistic methods // Matematiche (Catania). 1992. V. 47. №1. P. 25-61.

[23] P. G. Burke. R-matrix theory: some recent applications // Many-Body Atomic Physics / ed. J. J. Boyle, M. S. Pindzola. Cambridge: Cambridge Univ. Press, 1996. P. 305-348.

[24] A. P. Calderón. Cauchy integrals on Lipschitz curves and related operators // Proc. Natl. Acad. Sci. USA. 1977. V. 74. P. 1324-1327.

[25] A. P. Calderón. Boundary value problems for the Laplace equation in Lipschitzian domains // Recent Progress in Fourier Analysis. Proc. semin. El Escorial, 1983. Amsterdam: North-Holland, 1985. P. 33-48. (North-Holland Math. Stud. V. 111.)

[26] R. R. Coifman, A. McIntosh, Y. Meyer. L'intégrale de Cauchy définit un opérateur borné sur $L^{2}$ pour les courbes lipschitziennes // Ann. of Math. (2). 1982. V. 116. P. 361-387.

[27] Д. Колтон, Р. Кресс. Методы интегральных уравнений в теории рассеяния. М.: Мир, 1987.

[28] M. Costabel. Boundary integral operators on Lipschitz domains: elementary results // SIAM J. Math. Anal. 1988. V. 19. №3. P. 613-626.

[29] M. Costabel, W. L. Wendland. Strong ellipticity of boundary integral operators // J. Reine Angew. Math. 1986. V. 372. P. 34-63.

[30] Р. Курант, Д. Гильберт. Методы математической физики. Т. 1. М.-Л.: Гостехтеориздат, 1933.

[31] B. Dahlberg, G. Verchota. Galerkin methods for the boundary integral equations of elliptic equations in nonsmooth domains // Contemp. Math. 1990. V. 107. P. 39-60.

[32] B. Dahlberg, C. Kenig, G. Verchota. Boundary value problems for the systems of elastostatics in Lipschitz domains // Duke Math. J. 1988. V. 57. № 3. P. 795-818.

[33] B. Dahlberg, C. Kenig, J. Pipher, G. Verchota. Area integral estimates for higher order elliptic equations and systems // Ann. Inst. Fourier (Grenoble). 1997. V. 47. №5. P. $1425-1461$.

[34] G. David. Wavelets and Singular Integrals on Curves and Surfaces. Berlin: Springer-Verlag, 1991. (Lecture Notes in Math. V. 1465.)

[35] Z. Ding. A proof of the trace theorem of Sobolev spaces on Lipschitz domains // Proc. Amer. Math. Soc. 1996. V. 124. № 2. P. 591-600.

[36] Г.И. Эскин. Краевые задачи для эллиптических псевдодифференциальных уравнений. М.: Наука, 1973.

[37] E. Fabes. Layer potential methods for boundary value problems on Lipschitz domains // Potential Theory: Surveys and Problems. Berlin: Springer-Verlag, 1988. P. 55-80. (Lecture Notes in Math. V. 1344.)

[38] E. Fabes, M. Jodeit, N. Rivière. Potential techniques for boundary value problems on $C^{1}$ domains // Acta Math. 1978. V. 141. P. 165-186.

[39] E. Fabes, O. Mendez, M. Mitrea. Boundary layers on Sobolev-Besov spaces and Poisson's equation for the Laplacian in Lipschitz domains // J. Funct. Anal. 1998. V. 159. № 2. P. 323-368.

[40] W. Gao. Layer potentials and boundary value problems for elliptic systems in Lipschitz domains // J. Funct. Anal. 1991. V. 95. № 2. P. 377-399.

[41] И.Ц. Гохберг, М.Г. Крейн. Введение в теорию несамосопряженных операторов в гильбертовом пространстве. М.: Наука, 1965. 
[42] 3. Н. Голубева. Некоторые скалярные задачи дифракции и связанные с ними несамосопряженные операторы // Радиотехника и электроника. 1976. Т. 21. № 2. С. 219-227.

[43] P. Grisvard. Caractérisation de quelques espaces d'interpolation // Arch. Ration. Mech. Anal. 1967. V. 25. P. 40-63.

[44] P. Grisvard. Elliptic Problems in Nonsmooth Domains. Boston: Pitman, 1985.

[45] В. В. Грушин. О фундаментальных решениях гипоэллиптических уравнений // УМН. 1961. T. 16. № 4. C. $147-153$.

[46] Л. Хёрмандер. Анализ линейных дифференциальных операторов с частными производньми. Т. 3, 4. М.: Мир, 1987; 1988.

[47] В.Я. Иврий. О точных спектралњных асимптотиках для эллиптических операторов, действующих в расслоениях // Функц. анализ и его прил. 1982. Т. 16. № 2. С. 30-38.

[48] V. Ivrii. Precise spectral asymptotics for elliptic operators acting in fiberings over manifolds with boundary. Berlin: Springer-Verlag, 1984. (Lecture Notes in Math. V. 1100.)

[49] V. Ivrii. Microlocal Analysis and Precise Spectral Asymptotics. Berlin: Springer-Verlag, 1998.

[50] V. Ivrii. Sharp spectral asymptotics for operators with irregular coefficients // Internat. Math. Res. Notes. 2000. V. 22. P. 1155-1166.

[51] D. Jerison, C. Kenig. The Dirichlet problem in non-smooth domains // Ann. of Math. (2). 1981. V. 113. P. 367-382.

[52] D. Jerison, C. Kenig. Boundary value problems on Lipschitz domains // MAA Studies in Math. 1982. V. 23. P. 1-68.

[53] D. Jerison, C. Kenig. The inhomogeneous Dirichlet problem in Lipschitz domains // J. Funct. Anal. 1995. V. 130. № 1. P. 161-219.

[54] H.-C. Kaiser, H. Neidhardt, J. Rehberg. Macroscopic current induced boundary conditions for Schrödinger-type operators // Preprint 630. Berlin: Weierstrass-Institute for Applied Analysis and Stochastics, 2001.

[55] H. Kalf, U.-W. Schmincke, J. Walter, R. Wüst. On the spectral theory of Schrödinger and Dirac operators with strongly singular potentials // Spectral Theory and Differential Equations. Berlin: Springer-Verlag, 1975. P. 182-226. (Lecture Notes in Math. V. 448.)

[56] Т. Като. Теория возмущений линейных операторов. М.: Мир, 1972.

[57] В.Э. Кацнельсон. О сходимости и суммируемости рядов по корневым векторам некоторых классов несамосопряженных операторов // Кандидатская дисс. Харьков, 1967.

[58] В.Э. Кацнельсон. Об условиях базисности системы корневых векторов некоторых классов операторов // Функц. анализ и его прил. 1967. Т. 1. № 2. С. 39-51.

[59] C. Kenig. Recent progress on boundary-value problems on Lipschitz domains // Pseudodifferential Operators and Applications. Providence, RI: Amer. Math. Soc., 1985. P. 175-205. (Proc. Sympos. Pure Math. V. 43.)

[60] C. Kenig. Elliptic boundary value problems on Lipschitz domains // Beijing Lectures in Harmonic Analysis. Princeton, NJ: Princeton Univ. Press, 1986. P. 131-183. (Ann. of Math. Stud. V. 112.)

[61] C. Kenig. Harmonic Analysis Techniques for Second Order Elliptic Boundary Value Problems. Providence, RI: Amer. Math. Soc., 1994. (Regional Conf. Series in Math. № 83.)

[62] В.А. Кондратьев. Краевые задачи для эллиптических уравнений в областях с коническими или угловыми точками // Труды ММО. 1967. Т. 16. С. 209-292.

[63] В. А. Кондратьев, С. Д. Эйдельман. Об условиях на граничную поверхность в теории эллиптических граничных задач // Докл. АН СССР. 1979. Т. 246. № 4. С. 812-815.

[64] В.А. Кондратьев, О. А. Олейник. Краевые задачи для системы теории упругости в неограниченных областях. Неравенства Корна // УМН. 1988. Т. 43. № 5. С. 55-98.

[65] Г.П. Костометов. Об асимптотике спектра интегральных операторов с полярными ядрами // Вестник ЛГУ. 1977. № 13. С. 166-167.

[66] V.A. Kozlov. Traction boundary value problem for anizotropic elasticity in polyhedral domains // Russian J. Math. Phys. 2001. V. 8. № 3. P. 275-286. 
[67] V.A. Kozlov, V. G. Maz'ya, J. Rossmann. Elliptic Boundary Value Problems in Domains with Point Singularities. Providence, RI: Amer. Math. Soc., 1997. (Math. Surveys Monogr. V. 52.)

[68] V.A. Kozlov, V. G. Maz'ya, J. Rossmann. Spectral Problems Associated with Corner Singularities of Solutions to Elliptic Equations. Providence, RI: Amer. Math. Soc., 2001. (Math. Surveys Monogr. V. 85.)

[69] H. Kumano-go. Pseudo-Differential Operators. Cambridge, MA: MIT Press, 1974.

[70] В.Д. Купрадзе, Т. Г. Гегелиа, М.О. Башелейшвили, Т. В. Бурчуладзе. Трехмерные задачи математической теории упругости и термоупругости. М.: Наука, 1976.

[71] О.А. Ладыженская, Н. Н. Уральцева. Линейные и квазилинейные уравнения эллиптического типа. М.: Наука, 1964 (1-е изд.); 1973 (2-е изд.).

[72] В.Б. Лидский. О суммируемости рядов по главным векторам несамосопряженных операторов // Труды ММО. 1962. Т. 11. С. 3-35.

[73] J.-L. Lions. Espaces d'interpolation et domaines de puissances fractionnaires d'opérateurs // J. Math. Soc. Japan. 1962. V. 14. № 2. P. 233-241.

[74] Ж.-Л. Лионс, Э. Мадженес. Неоднородные граничные задачи и их приложения. М.: Мир, 1971.

[75] А. С. Маркус. О разложении по корневым векторам слабо возмущенного самосопряженного оператора // Докл. АН СССР. 1962. Т. 142. №3. С. 538-541.

[76] А. С. Маркус. Введение в спектральню теорию полиномиальых операторных пучков. Кишинев: Штиинца, 1986.

[77] А. С. Маркус, В.И. Мацаев. Операторы, порожденные полуторалинейными формами, и их спектральные асимптотики // Матем. исслед. 1981. Т. 61. С. 86-103.

[78] А.С. Маркус, В.И. Мацаев. О сходимости разложений по собственным векторам оператора, близкого к самосопряженному // Матем. исслед. 1981. Т. 61. С. 104-129.

[79] А. С. Маркус, В.И. Мацаев. Теоремы сравнения спектров линейных операторов и спектральные асимптотики // Труды ММО. 1982. Т. 45. С. 133-181.

[80] В. Г. Мазья, Т. О. Шапошникова. Мультипликаторы в пространствах дифференцируемых функций. Л.: Изд-во ЛГУ, 1986.

[81] R. Mazzeo, R. McOwen. Singular Sturm-Liouville theory on manifolds // J. Differential Equations. 2001. V. 176. № 2. P. 387-444.

[82] A. McIntosh. On representing closed accretive sesquilinear forms as $\left(A^{1 / 2} u, A^{* 1 / 2} v\right)$ // Nonlinear Partial Differential Equations and Their Applications. College de France Sem. V. III. Res. Notes Math. V. 70, 1982. P. 252-267.

[83] W. McLean. Strongly Elliptic Systems and Boundary Integral Equations. Cambridge: Cambridge Univ. Press, 2000.

[84] G. Métivier. Valeurs propres de problèmes aux limites elliptiques irrégulieres // Bull. Soc. Math. France. Suppl. Mém. 1977. № 51-52. P. 125-219.

[85] С. Г. Михлин. Курс математической физики. М.: Наука, 1968.

[86] D. Mitrea. The method of layer potentials for non-smooth domains with arbitrary topology // Integral Equations Operator Theory. 1997. V. 29. № 3. P. 320-338.

[87] D. Mitrea, M. Mitrea. General second order, strongly elliptic systems in low dimensional nonsmooth manifolds // Contemp. Math. 2001. V. 277. P. 61-86.

[88] D. Mitrea, M. Mitrea, J. Pipher. Vector potential theory on nonsmooth domains in $\mathbb{R}^{3}$ and applications to electromagnetic scattering // J. Fourier Anal. Appl. 1997. V. 3. № 2. P. 131-192.

[89] D. Mitrea, M. Mitrea, M. Taylor. Layer Potentials, the Hodge Laplacian, and Global Boundary Problems in Nonsmooth Riemannian Manifolds. Providence, RI: Amer. Math. Soc., 2001. (Mem. Amer. Math. Soc. № 713.)

[90] M. Mitrea. Dirichlet integrals and Gaffney-Friedrichs inequalities in convex domains // Forum Math. 2001. V. 13. № 4. P. 531-567.

[91] M. Mitrea, M. Taylor. Boundary layer methods for Lipschitz domains in Riemannian manifolds // J. Funct. Anal. 1999. V. 163. № 2. P. 181-251. 
[92] M. Mitrea, M. Taylor. Potential theory on Lipschitz domains in Riemannian manifolds: Sobolev-Besov results and the Poisson problem // J. Funct. Anal. 2000. V. 176. № 1. P. 1-79.

[93] Y. Miyazaki. The eigenvalue distribution of elliptic operators with Hölder continuous coefficients. II // Osaka Math. J. 1993. V. 30. № 2. P. 267-301.

[94] C. B. Morrey, Jr. Second-order elliptic systems of differential equations // Contributions to the Theory of Partial Differential Equations / ed. L. Bers et al. Princeton: Princeton Univ. Press, 1954. P. 101-159. (Ann. Math. Stud. № 33.)

[95] T. Muramatu. On Besov spaces and Sobolev spaces of generalized functions defined on a general region // Publ. Res. Inst. Math. Sci. 1974. V. 9. P. 325-396.

[96] F. J. Narcovich. Mathematical theory of the $R$ matrix. I. The eigenvalue problem. II. The $R$ matrix and its properties // J. Math. Phys. 1974. V. 15. № 10. P. 1626-1634; 1635-1642.

[97] Д. Г. Натрошвили. Исследование краевых и начально-краевых задач математической теории упругости и термоупругости для однородных анизотропных сред методом потенциала // Докторская дисс. Тбилиси, 1984.

[98] D. G. Natroshvili. Boundary integral equation method in the steady state oscillation problems for anisotropic bodies // Math. Methods Appl. Sci. 1997. V. 20. № 2. P. 95-119.

[99] S.A. Nazarov, B. A. Plamenevsky. Elliptic Problems in Domains with Piecewise Smooth Boundaries. Berlin: de Gruyter, 1994. (de Gruyter Exp. Math. V. 13.)

[100] J. Nečas. Sur les domaines du type $\mathscr{N} / /$ Czechoslovak Math. J. 1962. V. 12. P. 274-287.

[101] J. Nečas. Les méthodes directes en théorie des équations elliptiques. Paris / Prague: Masson / Academia, 1967.

[102] М.И. Нейман-Заде, А. А. Шкаликов. Операторы Шрёдингера с сингулярными потенциалами из пространств мультипликаторов // Матем. заметки. 1999. Т. 66. №5. C. $723-733$.

[103] L. Nirenberg. Remarks on strongly elliptic partial differential equations // Comm. Pure Appl. Math. 1955. V. 8. P. 649-675.

[104] O.A. Oleinik, A.S.Shamaev, G. A. Yosifian. Mathematical Problems in Elasticity and Homogenization. Amsterdam: North-Holland, 1992. (Stud. Math. Appl. V. 26.)

[105] В. И. Параска. Об асимптотике собственных и сингулярных чисел линейных операторов, повышающих гладкость // Матем. сб. 1965. Т. 68. № 4. С. 621-631.

[106] L. Payne, H. Weinberger. New bounds for solutions of second order elliptic partial differential equations // Pacific J. Math. 1958. V. 8. P. 551-573.

[107] Ш. Ремпель, Б.-В. Шульце. Теория индекса эллиптических краевых задач. М.: Мир, 1986.

[108] Р. Рокафеллар. Выпуклый анализ. М.: Мир, 1973.

[109] Г. В. Розенблюм, М.З. Соломяк, М.А.Шубин. Спектральная теория дифференциальных операторов // Итоги науки и техники. Совр. проблемы матем. Фунд. напр. Т. 64. Диф. уравнения с частными производными VII. М.: ВИНИТИ, 1989. С. 5-247.

[110] Yu. Safarov, D. Vassiliev. The Asymptotic Distribution of Eigenvalues of Partial Differential Operators. Providence, RI: Amer. Math. Soc., 1991. (Transl. Math. Monogr. V. 155.)

[111] G. Savaré. Regularity results for elliptic equations in Lipschitz domains // J. Funct. Anal. 1988. V. 152. № 1. P. 176-201.

[112] А. М. Савчук, А. А. Шкаликов. Операторы Штурма-Лиувилля с сингулярными потенциалами // Матем. заметки. 1999. Т. 66. №6. С. 897-912.

[113] M. Schechter. A generalization of the problem of transmission // Ann. Scuola Norm. Sup. Pisa. Ser. III, Sci. Fis. Mat. 1960. V. 14. P. 207-236.

[114] R. T. Seeley. Refinement of the functional calculus of Calderón and Zygmund // Konink. Nederl. Acad. Wetensch. Proc. Ser. A. 1965. V. 68. № 3. P. 521-531.

[115] R. T. Seeley. Singular integrals and boundary value problems // Amer. J. Math. 1966. V. 88. P. 781-809.

[116] R. T. Seeley. Interpolation in $L^{p}$ with boundary conditions // Studia Math. 1972. V. 44. P. $47-60$.

[117] В. С. Серов. О сходимости рядов Фурье по собственньм функциям оператора Шрёдингера с потенциалом Като // Матем. заметки. 2000. Т. 67. № 5. С. 755-763. 
[118] И. Стейн. Сингулярные интегралы и диффференциальные свойства функций. М.: Мир, 1973.

[119] R. Szmytkowski. Metoda $R$-macierzy dla równań Schrödingera i Diraca. Gdańsk: Politechnika Gdańska, 1999.

[120] R. H. Torres, G. V. Welland. The Helmholtz equation and transmission problems with Lipschitz interfaces // Indiana Univ. Math. J. 1993. V. 42. № 4. P. 1457-1485.

[121] Б. Р. Вайнберг. Принципы излучения, предельного поглощения и предельной амплитуды в общей теории уравнений с частными производными // УМН. 1966. Т. 21. № 3. C. $115-194$.

[122] Б. Р. Вайнберг. Асимптотические методы в уравнениях математической физики. М.: Изд-во МГУ, 1982.

[123] G. Verchota. Layer potentials and regularity for the Dirichlet problem for Laplace's equation in Lipschitz domains // J. Funct. Anal. 1984. V. 59. P. 572-611.

[124] G. Verchota. Remarks on 2nd order elliptic systems in Lipschitz domains // Operator Theory and Partial Differential Equations, Miniconf. Ryde/Aust., 1986. Proc. Centre Math. Anal. Austral. Nat. Univ. V. 14, 1986. P. 303-325.

[125] М.И. Вишик. О сильно эллиптических системах дифференциальных уравнений // Докл. АН СССР. 1950. Т. 74. № 5. С. 881-884.

[126] Н.Н.Войтович, Б.З. Каценеленбаум, А. Н. Сивов. Обобщенньй метод собственных колебаний в теории дифракции. М.: Наука, 1977.

[127] Х. Уитни. Геометрическая теория интегрирования. М.: ИЛ, 1960.

[128] L. Zielinski. Asymptotic distribution of eigenvalues for some elliptic operators with simple remainder estimates // J. Operator Theory. 1998. V. 39. № 2. P. 249-282.

[129] А. Зигмунд. Тригонометрические ряды. Т. 2. М.: Мир, 1965.

Московский институт электроники и математики

Поступила в редакцию

17.04.2002 